DESY 13-025

\title{
Nucleon form factors, generalized parton distributions and quark angular momentum
}

\author{
Markus Diehl \\ Deutsches Elektronen-Synchroton DESY, 22603 Hamburg, Germany \\ Peter Kroll \\ Fachbereich Physik, Universität Wuppertal, 42097 Wuppertal, Germany \\ Institut für Theoretische Physik, Universität Regensburg, 93040 Regensburg, Germany
}

\begin{abstract}
We extract the individual contributions from $u$ and $d$ quarks to the Dirac and Pauli form factors of the proton, after a critical examination of the available measurements of electromagnetic nucleon form factors. From this data we determine generalized parton distributions for valence quarks, assuming a particular form for their functional dependence. The result allows us to study various aspects of nucleon structure in the valence region. In particular, we evaluate Ji's sum rule and estimate the total angular momentum carried by valence quarks at the scale $\mu=2 \mathrm{GeV}$ to be $J_{v}^{u}=0.230_{-0.024}^{+0.009}$ and $J_{v}^{d}=-0.004_{-0.016}^{+0.010}$.
\end{abstract}




\section{Contents}

1 Introduction $\quad 3$

2 Basics and notation 3

3 Data selection 4

3.1 Proton form factors . . . . . . . . . . . . . . . . . . . 5

3.2 Neutron form factors . . . . . . . . . . . . . . . . . . . . 7

3.3 A note on the dipole parameterization . . . . . . . . . . . . . 9

3.4 A global form factor fit . . . . . . . . . . . . . . . . 9

4 Strangeness

5 Interpolated data 14

5.1 Determination of flavor form factors . . . . . . . . . . . . . . . 14

5.2 Simple fits to form factors . . . . . . . . . . . . . . . . . 17

6 GPD fit 20

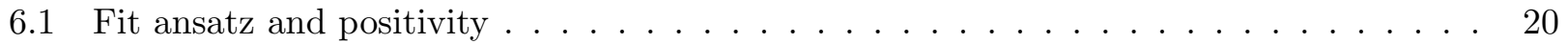

6.2 Selection of parton densities . . . . . . . . . . . . . . . . 21

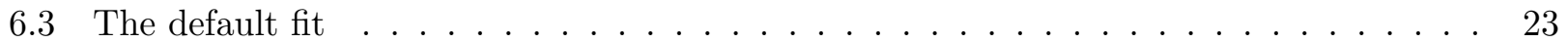

6.4 Variations of the default fit . . . . . . . . . . . . . . . 29

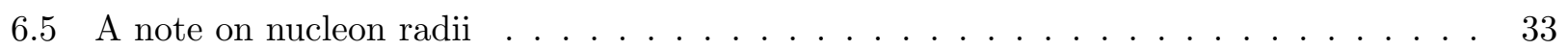

7 Properties of the fit $\quad 34$

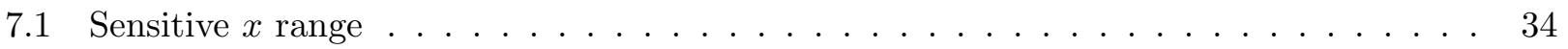

7.2 Mellin moments and Ji's sum rule . . . . . . . . . . . . . . 37

7.3 Impact parameter distributions . . . . . . . . . . . . . . . . . . . 40

8 Applications 41

8.1 Axial form factor . . . . . . . . . . . . . . . . . . . 41

8.2 Compton form factors . . . . . . . . . . . . . . . . . 42

8.3 Chromodynamic lensing and the Sivers distribution . . . . . . . . . . . . 43

8.4 Skewness dependence . . . . . . . . . . . . . . . . . . 45

9 Summary 47

A Tables of form factors

$\begin{array}{lll}\text { B Matrices for computing fit errors } & 50\end{array}$

References 54 


\section{Introduction}

Together with parton distributions, electromagnetic form factors are among the most important quantities that provide information about the internal structure of the nucleon. Their experimental determination has entered the realm of precision physics. Generalized parton distributions (GPDs) combine and enlarge the different types of information contained in ordinary parton densities (PDFs) and form factors, but they remain much less well known experimentally. After pioneering measurements at DESY and Jefferson Lab, the upcoming energy upgrade at Jefferson Lab will significantly advance the determination of GPDs in the valence quark region, whereas measurements at COMPASS will explore the region of sea quarks and gluons with momentum fractions between $10^{-2}$ and $10^{-1}$. For reviews of the many facets of GPDs we refer to [1, 2, 3, 4,

GPDs can be extracted from hard exclusive processes like deeply virtual Compton scattering and meson production. In complement, one can constrain the GPDs for valence quarks indirectly via the sum rules that connect them with electromagnetic form factors. This requires an ansatz for the functional form of the GPDs and in this sense is intrinsically model dependent, but on the other hand it can reach values of the invariant momentum transfer $t$ much larger than what can conceivably be measured in hard exclusive scattering. We performed such an indirect determination some time ago [5]. Since then, there have been significant improvements in the experimental determination of the electromagnetic form factors, and we find it timely to investigate how this progress impacts on the extraction of GPDs and of important quantities such as the total angular momentum carried by quarks in the proton. This is the purpose of the present work.

In section 2 we briefly recall some essentials about form factors and GPDs and introduce our notation. A critical discussion of the form factor data used in our analysis is given in section 3 . where we also provide a simple and precise parameterization of the selected data. In section 4 we estimate the contribution of strange quarks to the form factors. Section 5 describes how we combine the experimental results on proton and neutron form factors in order to extract Dirac and Pauli form factors for individual quark flavors, which are most closely connected with GPDs. Our fit of the GPDs to the form factor data, including a number of variants that allow us to investigate systematic uncertainties, is described in sections 6 and 7. In particular, we evaluate Ji's sum rule and thus obtain an estimate for the total angular momentum carried by $u$ and by $d$ quarks minus the corresponding contribution from antiquarks. Using our extracted GPDs, we explore in section 8 a number of further connections, namely the axial form factor, wide-angle Compton scattering, chromodynamic lensing and GPDs at nonzero skewness. We summarize our findings in section 9 and list various numerical results in two appendices.

\section{Basics and notation}

To begin with, let us recall some basics about the electromagnetic form factors of the nucleon. Experimental results are typically expressed in terms of the Sachs form factors $G_{M}^{p}(t), G_{E}^{p}(t)$ and $G_{M}^{n}(t), G_{E}^{n}(t)$, where $t$ is the squared momentum transfer to the proton. Several measurements determine the ratio of electric and magnetic form factors, which is commonly written as

$$
R^{i}(t)=\mu_{i} G_{E}^{i}(t) / G_{M}^{i}(t)
$$

with $i=p, n$ for the proton and the neutron. The magnetic moments $\mu_{p}$ and $\mu_{n}$ normalize this ratio to unity at $t=0$.

For convenience the magnetic form factors are often divided by the conventional dipole form

$$
G_{\text {dipole }}^{i}(t)=\frac{\mu_{i}}{\left[1-t /\left(0.71 \mathrm{GeV}^{2}\right)\right]^{2}}
$$

with $i=p, n$. Plotting this ratio allows one to discern details in the data over a wide range of $t$, since the ratios $G_{M}^{i} / G_{\text {dipole }}^{i}$ show only a mild variation, unlike the form factors themselves.

The Dirac and Pauli form factors, $F_{1}^{i}$ and $F_{2}^{i}$, are related to the Sachs form factors by

$$
G_{M}^{i}=F_{1}^{i}+F_{2}^{i}, \quad G_{E}^{i}=F_{1}^{i}+\frac{t}{4 m^{2}} F_{2}^{i},
$$


where $m$ is the nucleon mass and again $i=p, n$. One can further decompose

$$
\begin{aligned}
& F_{i}^{p}=e_{u} F_{i}^{u}+e_{d} F_{i}^{d}+e_{s} F_{i}^{s}, \\
& F_{i}^{n}=e_{u} F_{i}^{d}+e_{d} F_{i}^{u}+e_{s} F_{i}^{s},
\end{aligned}
$$

where $F_{i}^{q}$ denotes the contribution from quark flavor $q$ to the form factor $F_{i}^{p}$ of the proton. Here $i=1,2$ and $e_{q}$ is the electric charge of the quark in units of the positron charge. It is instructive to rewrite (4) as

$$
\begin{aligned}
& 2 F_{i}^{p}+F_{i}^{n}=F_{i}^{u}-F_{i}^{s}, \\
& 2 F_{i}^{n}+F_{i}^{p}=F_{i}^{d}-F_{i}^{s} .
\end{aligned}
$$

To the extent that the strangeness contributions $F_{1}^{s}$ and $F_{2}^{s}$ can be neglected, one can hence reconstruct the form factors for $u$ and $d$ quarks from the electromagnetic form factors alone. We will return to the issue of strangeness form factors in section 4. For brevity we will refer to the set of $F_{i}^{q}$ as "flavor form factors" in this work. We will also use self-explaining abbreviations

$$
F_{i}^{u-s}=F_{i}^{u}-F_{i}^{s}, \quad F_{i}^{u+d}=F_{i}^{u}+F_{i}^{d}
$$

etc. for linear combinations of these form factors.

The flavor form factors can be written in terms of GPDs at zero skewness. For each quark flavor we have the sum rules

$$
\begin{aligned}
& F_{1}^{q}(t)=\int_{0}^{1} \mathrm{~d} x H_{v}^{q}(x, t), \\
& F_{2}^{q}(t)=\int_{0}^{1} \mathrm{~d} x E_{v}^{q}(x, t)
\end{aligned}
$$

with

$$
\begin{aligned}
H_{v}^{q}(x, t) & =H^{q}(x, 0, t)+H^{q}(-x, 0, t), \\
E_{v}^{q}(x, t) & =E^{q}(x, 0, t)+E^{q}(-x, 0, t),
\end{aligned}
$$

where $H^{q}(x, \xi, t)$ and $E^{q}(x, \xi, t)$ denote the proton GPDs for unpolarized quarks of flavor $q$ in the standard notation 2]. The combinations (8) correspond to the difference of quarks and antiquarks (as it must be for the electromagnetic form factors) and in this sense can be called "valence GPDs". For positive $x$ one recovers the usual quark and antiquark densities as $H^{q}(x, 0,0)=$ $q(x)$ and $H^{q}(-x, 0,0)=-\bar{q}(x)$.
We will also need the combination

$$
\widetilde{H}_{v}^{q}(x, t)=\widetilde{H}^{q}(x, 0, t)-\widetilde{H}^{q}(-x, 0, t)
$$

for the difference of longitudinally polarized quarks and antiquarks, as well as the antiquark distributions

$$
\begin{aligned}
H^{\bar{q}}(x, t) & =-H^{q}(-x, 0, t), \\
E^{\bar{q}}(x, t) & =-E^{q}(-x, 0, t), \\
\widetilde{H}^{\bar{q}}(x, t) & =\widetilde{H}^{q}(-x, 0, t) .
\end{aligned}
$$

With these definitions, the isovector axial form factor of the nucleon can be written as

$$
\begin{aligned}
F_{A}(t)= & \int_{0}^{1} \mathrm{~d} x\left[\widetilde{H}_{v}^{u}(x, t)-\widetilde{H}_{v}^{d}(x, t)\right] \\
& +2 \int_{0}^{1} \mathrm{~d} x\left[\widetilde{H}^{\bar{u}}(x, t)-\widetilde{H}^{\bar{d}}(x, t)\right] .
\end{aligned}
$$

The sea quark contribution does not drop out in this sum rule since the axial form factor has positive charge parity and thus corresponds to the sum and not the difference of quark and antiquark contributions. The value of $F_{A}$ at $t=0$, the axial charge, is well known from $\beta$-decay experiments.

\section{Data selection}

The determination of the electromagnetic nucleon form factors has not only a long history but remains at the forefront of experimental research. With quoted uncertainties typically in the percent region, the consistency between different measurements and the control of the theory underlying them have become nontrivial issues, as we shall see. In this section we discuss the selection of data used in our subsequent analysis and point out open problems and discrepancies between data sets. Earlier overviews and discussions of form factor data can be found in [6, 8, 7], and for $G_{E}^{n}$ also in [27.

A synopsis of the default data set that we use in later sections is given in table 1. Several of these data do not have separated statistical and systematic errors. To have a uniform treatment, we add those errors in quadrature for the data sets where they are available. 


\begin{tabular}{ccc}
\hline & $-t\left[\mathrm{GeV}^{2}\right]$ & references \\
\hline$G_{M}^{p}$ & $0.017-31.2$ & {$[9]$} \\
\cline { 2 - 3 }$R^{p}$ & $0.069-0.138$ & {$[9]$} \\
& $0.246-8.49$ & {$[10]-[19]$} \\
\cline { 2 - 3 }$G_{M}^{n}$ & $0.071-0.235$ & {$[20,21]$} \\
& $0.1-4.77$ & {$[22]-[24]$} \\
\cline { 2 - 3 }$R^{n}$ & $0.142-3.41$ & {$[25]-[34]$} \\
$G_{E}^{n}$ & $0.0389-1.644$ & {$[35]$} \\
$r_{E n}^{2}$ & 0 & {$[36]$} \\
\hline
\end{tabular}

Table 1: Overview of our default data set. More information about the data on $R^{p}, G_{M}^{n}$ and $R^{n}$ is given in tables 2, 3 and 4 .

\subsection{Proton form factors}

One of the main observables for the extraction of the proton form factors is the unpolarized elastic $e p$ cross section, from which $G_{M}^{p}$ and $G_{E}^{p}$ may be obtained by a Rosenbluth separation. About a decade ago it has become evident that the effects of two-photon exchange are substantial in this extraction method [37], especially for $G_{E}^{p}$ but at the precision level also for $G_{M}^{p}$. Two-photon exchange must hence be described accurately, which is nontrivial because it involves the proton structure in a way that is even more complex than for the onephoton exchange term from which one wants to extract the form factors. For a review of this subject we refer to [38].

A second method uses the correlation between the polarizations of the beam electron and either the proton target or the scattered proton, i.e. the processes $\vec{p}\left(\vec{e}, e^{\prime} p\right)$ or $p\left(\vec{e}, e^{\prime} \vec{p}\right)$. This is typically found to be less sensitive to two-photon effects. However, it gives access only to the ratio $R^{p}$, so that information from the $e p$ cross section remains indispensable for the separate determination of $G_{M}^{p}$ and $G_{E}^{p}$.

There exist several global analyses that combine Rosenbluth separation and polarization data, plus in some cases the ratio of $e^{+} p$ and $e^{-} p$ cross sections. Most of them include a parameterization of two-photon exchange terms, whose parameters are fitted to data. This approach has been taken e.g. in [39, 40, 41, 42]. By contrast, the analy-
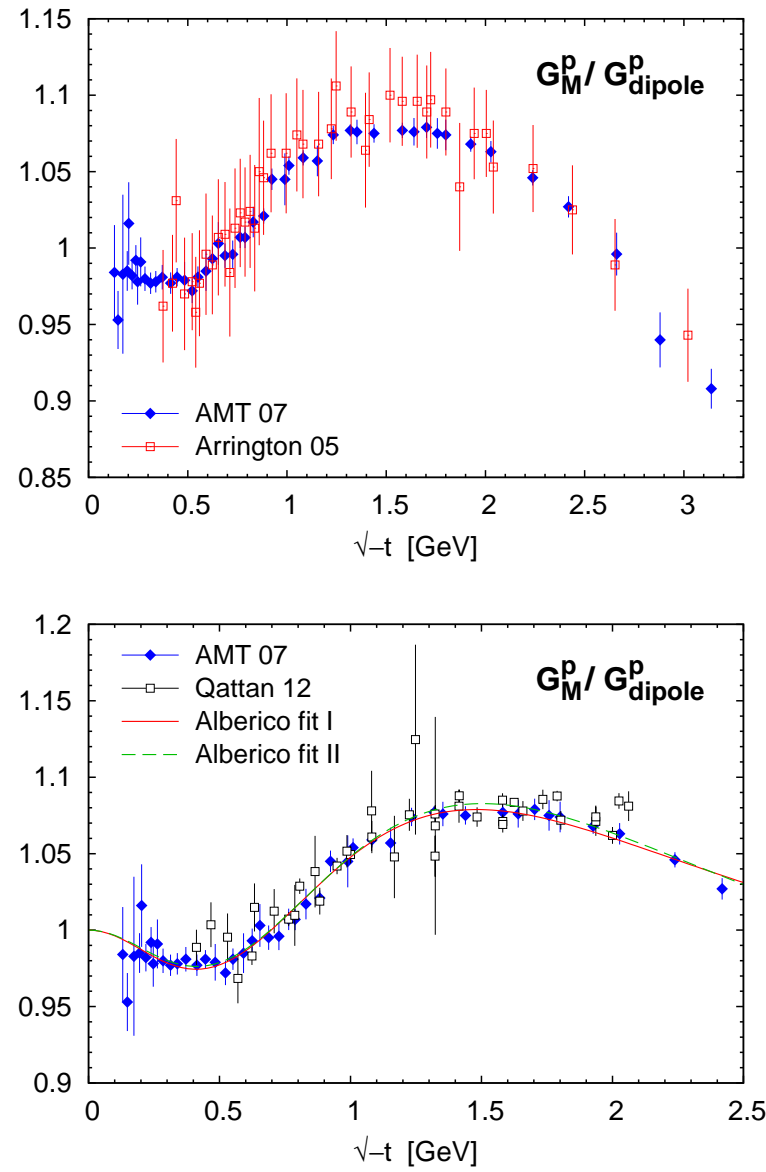

Figure 1: Data for $G_{M}^{p}$ from the global analyses AMT 07 [9], Arrington 05 [39] and Qattan 12 [42], along with two fits from the global analysis by Alberico et al. [40]. The form factor is divided by the dipole parameterization (2).

sis in [9] uses two-photon exchange terms calculated in dynamical models, including an estimate of their uncertainties. A comparative discussion of the different methods can be found in [41].

For our data set we use the $G_{M}^{p}$ results from the analysis [9] of Arrington, Melnitchouk and Tjon (hereafter referred to as AMT 07). This set covers the $t$ range from 0.007 to $31.2 \mathrm{GeV}^{2}$. We omit the two data points with the lowest $t$ values, which have huge errors and would not affect any our fits (but spoil the legibility of plots), and remain with data in the range from 0.017 to $31.2 \mathrm{GeV}^{2}$.

A comparison of the results for $G_{M}^{p}$ extracted in [9, 39, 42] is shown in figure 1, as well as two fits proposed in [40]. Here and in the following 
we plot form factors and their ratios against $\sqrt{-t}$ rather than against the more common variable $-t$, which permits a clearer view of the data at low $t$, where the density of measurements is highest. We observe that the error bars of AMT 07 are significantly smaller than those of [39] and [42, which reflects that in [39, 42] the size of two-photon exchange effects was fitted to the data rather than provided as an external, albeit model dependent, input. Given the good agreement between AMT 07 and the other analyses, including the central fit curves of [40], we think that the AMT 07 data may be taken as a representative of the current best knowledge of $G_{M}^{p}$. We will later cross check our results by using instead the values for $G_{M}^{p}$ obtained in [39] (Arrington 05), see section 6.4.

Let us now turn to the electric proton form factor. Rather than the values from the above global fits, we select in this case results for the ratio $R^{p}$ measured with the recoil polarization method or with a polarized proton target, i.e. with $p\left(\vec{e}, e^{\prime} \vec{p}\right)$ or $\vec{p}\left(\vec{e}, e^{\prime} p\right)$. The recoil polarization data cover a range of $-t$ from 0.246 to $8.49 \mathrm{GeV}^{2}$, and the polarized proton data from 0.162 to $1.51 \mathrm{GeV}^{2}$. To have some coverage at lower $-t$, we use in addition the values of $R^{p}$ with $-t=0.069,0.098$ and $0.138 \mathrm{GeV}^{2}$ from the analysis of AMT 07 [9].

An overview of the data sets is given in table 2, and the corresponding values of $R^{p}$ are shown in figure 2. We see that for $\sqrt{-t}$ between 0.5 and $0.9 \mathrm{GeV}$ the recent data from JLab Hall A (Paolone 10, Ron 11 and Zhan 11) show a clear systematic discrepancy with earlier data. Let us emphasize that this discrepancy concerns not only the polarized target data from MIT Bates (Crawford 07) but also the two data points of the Hall A recoil polarization measurement of Punjabi 05 in this $t$ range, as well as older recoil polarization data (which have however relatively large errors). We also note a discrepancy at $\sqrt{-t} \approx 1.2 \mathrm{GeV}$ between polarized target data (Jones 06) and recoil polarization (Punjabi 05), which is however less significant at the scale of the errors. To the best of our knowledge, the origin of these discrepancies is currently not understood. We sincerely hope that future investigations will clarify the experimental situation, which is quite unsatisfactory as it stands.
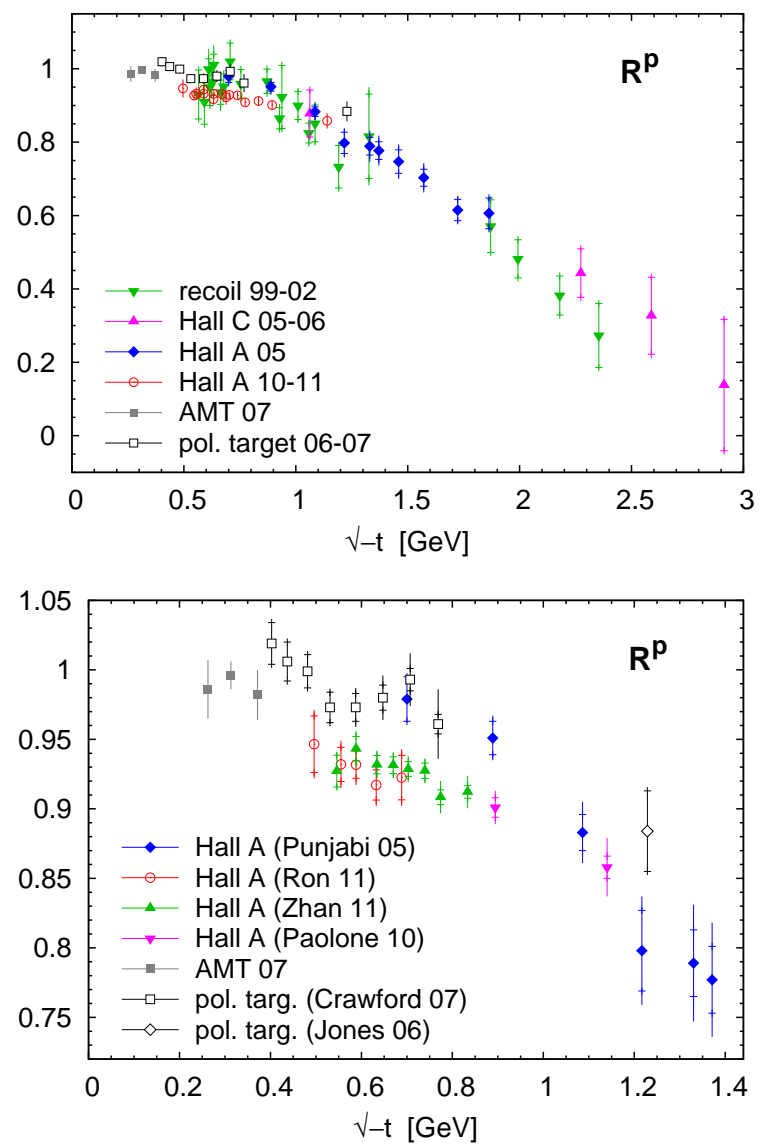

Figure 2: Results for $R^{p}$ from the data sets in table 2. The polarized target data (empty white squares or diamonds) are not used in our default data set. Inner error bars indicate statistical uncertainties when those are given separately.

Including all of the above data in least-square fits would not be useful in our opinion, since without modifications this fitting method is not designed to cope with manifestly inconsistent data sets. Lacking better criteria, we have chosen to include the more recent measurements in our default data set, which is composed of all recoil polarization data in table 2 and the three points of AMT 07 mentioned above. Note that this set still includes a tension in the data, since we have not removed the two points of Punjabi 05 with $\sqrt{-t}$ below $0.9 \mathrm{GeV}$. In section 6.4 we will investigate as an alternative the data set obtained by removing the measurements of Paolone 10, Ron 11 and Zhan 11 and by adding the polarized target data of Jones 06 and Crawford 07. 


\begin{tabular}{lcllll}
\hline \multicolumn{5}{c}{$R^{p}=\mu_{p} G_{E}^{p} / G_{M}^{p}$} \\
\hline process & $-t\left[\mathrm{GeV}^{2}\right]$ & \multicolumn{1}{c}{ reference } & facility & remarks \\
\hline$p\left(\vec{e}, e^{\prime} \vec{p}\right)$ & $0.38-0.50$ & Milbrath 99 & {$[10]$} & MIT Bates & \\
& $0.373-0.441$ & Pospischil 01 & {$[11]$} & MAMI A1 & \\
& $0.32-1.76$ & Gayou 01 & {$[12]$} & JLab Hall A & \\
& $3.50-5.54$ & Gayou 02 & {$[13]$} & JLab Hall A & \\
& $0.49-3.47$ & Punjabi 05 & {$[14]$} & JLab Hall A & \\
& 1.13 & MacLachlan 06 & {$[15]$} & JLab Hall C & \\
& $5.17-8.49$ & Puckett 10 & {$[16]$} & JLab Hall C & \\
\cline { 2 - 5 } & $0.8,1.3$ & Paolone 10 & {$[17]$} & JLab Hall A & \\
& $0.246-0.474$ & Ron 11 & {$[18]$} & JLab Hall A & updates $[43]$ \\
& $0.298-0.695$ & Zhan 11 & {$[19]$} & JLab Hall A & \\
\hline$\vec{p}\left(\vec{e}, e^{\prime} p\right)$ & 1.51 & Jones 06 & {$[44$} & JLab Hall C & \\
& $0.162-0.591$ & Crawford 07 & {$[45]$} & MIT Bates & \\
\hline
\end{tabular}

Table 2: Measurements of $R^{p}$ obtained with recoil polarization or with a polarized target (last two rows).

\subsection{Neutron form factors}

The magnetic form factor of the neutron can be determined from the cross section ratio of the quasi-elastic processes $d\left(e, e^{\prime} n\right)$ and $d\left(e, e^{\prime} p\right)$, and also from scattering polarized electrons on polarized ${ }^{3} \mathrm{He}$. The relevant data sets are listed in table 3 and shown in figure 3 . We do not use results of the MIT Bates measurement [48, 49] of ${ }^{3} \overrightarrow{\mathrm{He}}\left(\vec{e}, e^{\prime}\right)$, which has rather large errors and would not influence our analysis.

Looking at the deuterium measurements at $\sqrt{-t}$ just below $1 \mathrm{GeV}$, we observe an abrupt change between the data of Anklin 98 and Kubon 02 on one hand and the Hall B data (Lachniet 09) on the other. We do not consider this a physically plausible behavior and suspect a consistency problem between these measurements (which were using the same process), but we have not found any discussion of this issue in the literature. We also observe that the two data points of Anklin 98 for $\sqrt{-t}$ between 0.7 and $0.8 \mathrm{GeV}$ are systematically higher than the data of Anderson 07, which connect smoothly with those of Hall B. Since the Anderson 07 points are extracted from ${ }^{3} \mathrm{He}$ and thus have entirely different theoretical and sys- tematic uncertainties than the Hall B measurement, we consider this as a strong argument in favor of the more recent data. We hence decided to discard the points with $-t=0.504,0.652$ and $0.784 \mathrm{GeV}^{2}$ from Anklin 98 and the points with $-t=0.359$ and $0.894 \mathrm{GeV}^{2}$ from Kubon 02. We keep, however, the point with $-t=0.235 \mathrm{GeV}^{2}$ from Anklin 98 and the points with $-t=0.071$ and $0.125 \mathrm{GeV}^{2}$ from Kubon 02 , which are consistent with other data in the same $t$ region.

We remark that the global fit by Kelly [50] used the full data sets of Anklin 98 and Kubon 02, whereas the results of Anderson 07 and Lachniet 09 were not available at the time of that fit. As a consequence, the Kelly parameterization for $G_{M}^{n}$ faithfully reproduces the older data but is in clear conflict with the newer ones, as seen in figure 3 .

The electric neutron form factor $G_{E}^{n}$ has been extracted from a series of polarization measurements using deuterium or ${ }^{3} \mathrm{He}$, which are compiled in table 4. In the approximation of vanishing final state interactions, these experiments directly measure $G_{E}^{n} / G_{M}^{n}$ and obtain $G_{E}^{n}$ by using a value for $G_{M}^{n}$ as external input. This is problematic since the used values are not always in good agreement with the current experimental deter- 


\begin{tabular}{lcllll}
\hline \multicolumn{5}{c}{$G_{M}^{n}$} \\
\hline process & $-t\left[\mathrm{GeV}^{2}\right]$ & \multicolumn{2}{c}{ reference } & facility & remarks \\
\hline$d\left(e, e^{\prime} n\right), d\left(e, e^{\prime} p\right)$ & 0.111 & Anklin 94 & {$[22]$} & NIKHEF, PSI & \\
& $0.235-0.784$ & Anklin 98 & {$[20]$} & MAMI, PSI & used partially \\
& $0.071-0.894$ & Kubon 02 & {$[21]$} & MAMI, PSI & used partially \\
& $0.985-4.773$ & Lachniet 09 & {$[23]$} & JLab Hall B & \\
\hline${ }^{3} \overrightarrow{\mathrm{He}}\left(\vec{e}, e^{\prime}\right)$ & $0.1-0.6$ & Anderson 07 & {$[24$} & JLab Hall A & updates [46, 47] \\
\hline
\end{tabular}

Table 3: Data sets for the determination of $G_{M}^{n}$.

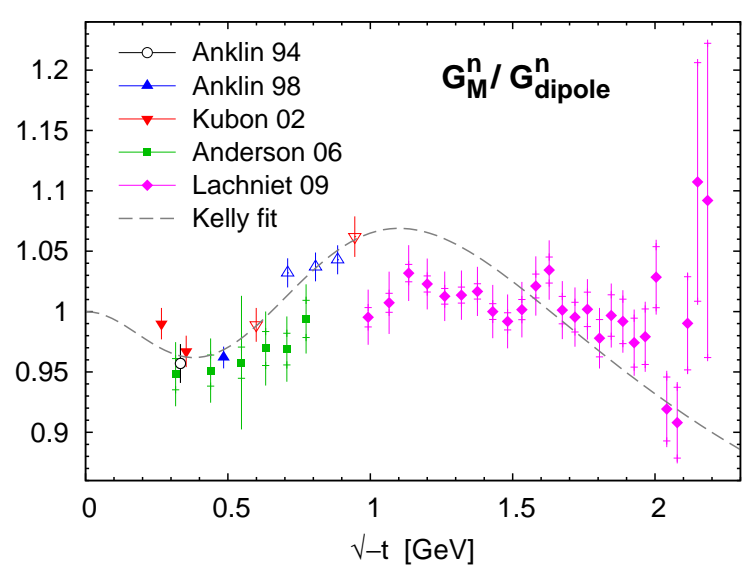

Figure 3: Data for $G_{M}^{n}$ divided by the dipole parameterization (2). The data points with open triangles (Anklin 98 and Kubon 02) are not used in our fits. Inner error bars indicate statistical uncertainties.

mination of $G_{M}^{n}$, especially if they are computed from the dipole parameterization (2) or from the Kelly parameterization [50]. To circumvent this bias, we use $R^{n}$ as input for our data selection; values of $G_{E}^{n}$ then result from our selection and analysis of the $G_{M}^{n}$ data. The papers [27, 31, 34] directly quote results for $R^{n}$, whereas in the other cases we have calculated $R^{n}$ from the quoted results for $G_{E}^{n}$, using the $G_{M}^{n}$ values and their errors as specified in the experimental papers. The resulting values and errors of $R^{n}$ are given in appendix A for reference.

As an independent source of information we take the values of $G_{E}^{n}$ extracted from the deuteron quadrupole form factor in Schiavilla 01 [35], which

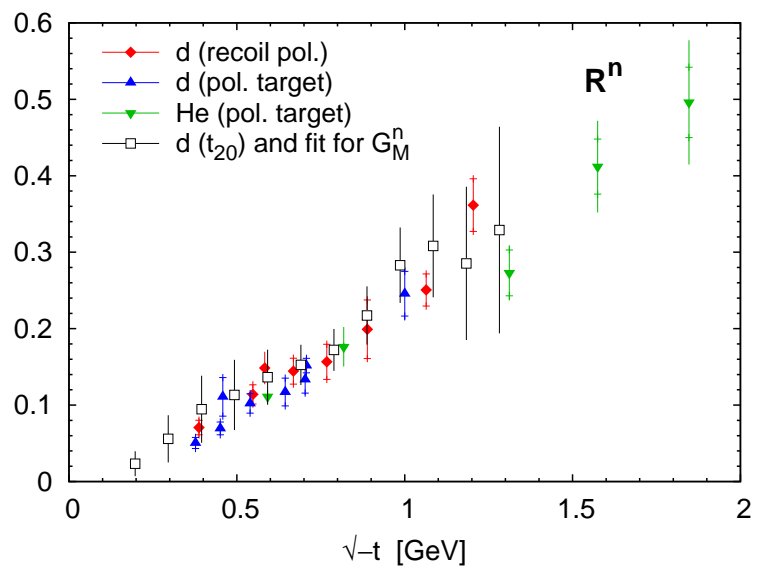

Figure 4: Data for $R^{n}$. The open (white) symbols correspond to data for $G_{E}^{n}$ together with $G_{M}^{n}$ taken from the fit described in section 3.4. Inner error bars indicate statistical uncertainties.

uses as input the measurement of the polarization observable $t_{20}$ and provides data points with $-t$ from 0.0389 to $1.644 \mathrm{GeV}^{2}$. These data have relatively large errors and are shown in figure 7 below. Taking the fit for $G_{M}^{n}$ that will be described in section 3.4, we can compute $R^{n}$ from these data. As can be seen in figure 4 , the result agrees well with $R^{n}$ extracted from the the measurements in table 4.

We note that the smallest value of $\sqrt{-t}$ for which we have a precise determination of $R^{n}$ (from Herberg 99 and Geis 08 ) is about $0.4 \mathrm{GeV}$. It is therefore highly welcome that entirely independent information about the small- $t$ behavior of $G_{E}^{n}$ is provided by the squared charge radius of 


\begin{tabular}{lcllll}
\hline \multicolumn{5}{c}{$R^{n}=\mu_{n} G_{E}^{n} / G_{M}^{n}$} \\
\hline process & $-t\left[\mathrm{GeV}^{2}\right]$ & \multicolumn{2}{c}{ reference } & facility & remarks \\
\hline$d\left(\vec{e}, e^{\prime} \vec{n}\right) p$ & $0.15,0.34$ & Herberg 99 & {$[25]$} & MAMI A1 & \\
& $0.3-0.79$ & Glazier 05 & {$[26]$} & MAMI A1 & updates [51] \\
& $0.447-1.45$ & Plaster 06 & {$[27]$} & Jlab Hall C & updates [52] \\
\hline$\vec{d}(\vec{e}, e n) p$ & 0.21 & Passchier 99 & {$[28$} & NIKHEF & \\
& 0.495 & Zhu 01 & {$[29]$} & JLab Hall C & \\
& $0.5,1.0$ & Warren 04 & {$[30]$} & JLab Hall C & \\
& $0.142-0.415$ & Geis 08 & {$[31]$} & MIT Bates & \\
\hline${ }^{3} \overrightarrow{\mathrm{He}}\left(\vec{e}, e^{\prime} n\right)$ & 0.67 & Bermuth 03 & {$[32]$} & MAMI A1 & updates [53] \\
& 0.35 & Rohe 05 & {$[33]$} & MAMI A3 & updates [54, 55] \\
& $1.72-3.41$ & Riordan 10 & {$[34]$} & JLab Hall A & \\
\hline
\end{tabular}

Table 4: Data sets for the determination of the neutron form factor ratio $R^{n}$.

the neutron, which is defined by

$$
r_{n E}^{2}=\left.6 \frac{\mathrm{d} G_{E}^{n}}{\mathrm{~d} t}\right|_{t=0}
$$

and can be measured in scattering a neutron beam off the shell electrons in a nuclear target. We use the value quoted in the 2012 Review of Particle Physics [36],

$$
r_{n E}^{2}=-(0.1161 \pm 0.0022) \mathrm{fm}^{2},
$$

which has been stable since its first listing in the 2002 edition of the same review. In principle we might have also included in our data selection the electric charge radius of the proton as determined from atomic physics, but the present controversy concerning the value of this radius prevented us from following this path. We will briefly discuss this in section 6.5.

\subsection{A note on the dipole parameteriza- tion}

When plotting $G_{M}^{p}$ and $G_{M}^{n}$ we follow common practice and divide by the dipole parameterization

$$
F(t)=\frac{F(0)}{\left(1-t / M_{\mathrm{dip}}^{2}\right)^{2}}
$$

of these form factors. As already emphasized, this is a matter of pure convenience, with the value
$M_{\text {dip }}^{2}=0.71 \mathrm{GeV}^{2}$ fixed by convention. As is evident from figures 1 and 3 , neither $G_{M}^{p}$ nor $G_{M}^{n}$ is particularly well described by this parameterization.

One may ask whether a good description of these form factors can be achieved with a different value of the dipole mass, at least in a certain range of $t$. To investigate this question, we define the effective dipole mass

$$
M_{\mathrm{eff}}^{2}(t)=\frac{-t}{\sqrt{F(0) / F(t)}-1},
$$

which is $t$ independent and equal to the dipole mass for a form factor with the shape (14). From the plots of this quantity in figure 5 we see that within their currently known precision, neither of the magnetic form factors is well represented by a dipole form in any interval that starts at $t=$ 0 . Somewhat amusingly, the dipole law with its conventional mass value approximately describes $G_{M}^{n}$ in the region of $\sqrt{-t}$ between 1 and $2 \mathrm{GeV}$, as is already visible in figure 3 .

\subsection{A global form factor fit}

Although the main goal of our study is the determination of the GPDs $H_{v}^{q}$ and $E_{v}^{q}$ from the form factors with the help of the sum rules (7) (see section 6), there is some interest in having a simple parameterization of the form factor data. This 

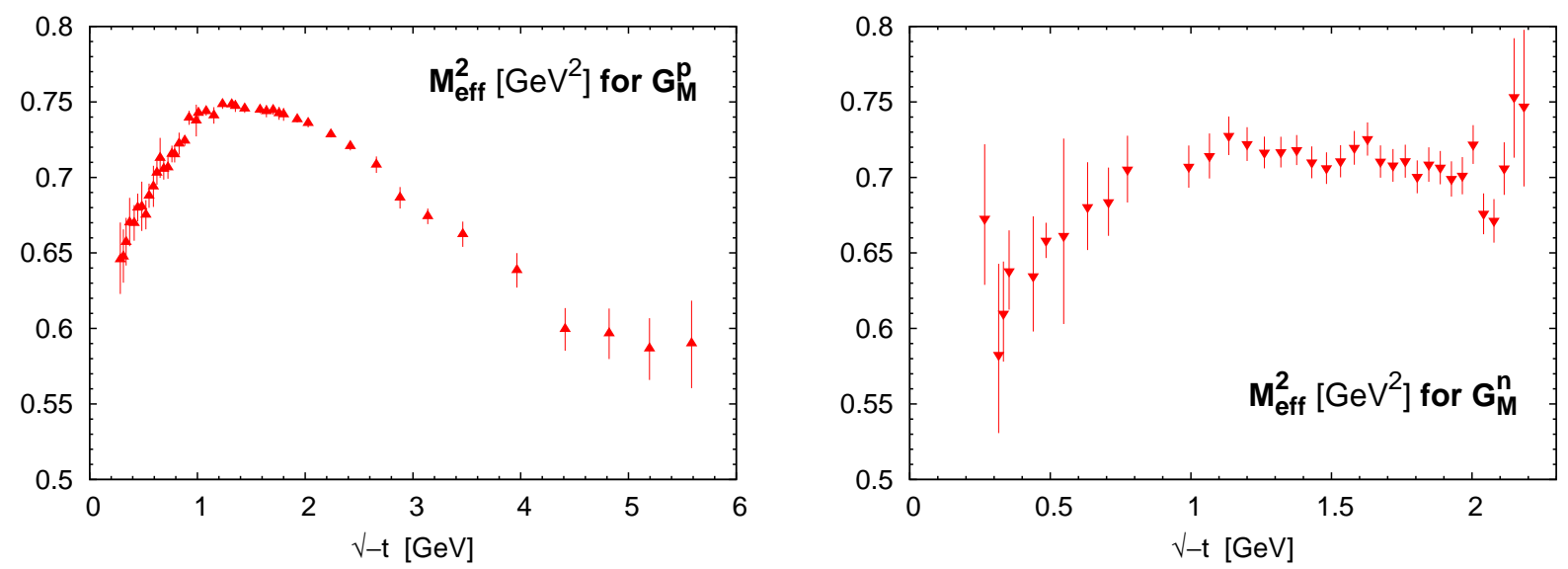

Figure 5: Effective dipole masses as defined in 15 for $G_{M}^{p}$ and $G_{M}^{n}$, evaluated from our data set.

parameterization can serve as a baseline for comparison with our GPD fits, for the interpolation of data or for the convenient evaluation of cross sections. An example for the latter is the evaluation of the Bethe-Heitler process, which plays a special role in connection with deeply virtual Compton scattering.

We find that a good representation of the data is possible if we represent the form factor combinations (5) as the product of two fractional power laws:

$$
F_{i}^{q-s}(t)=\frac{F_{i}^{q-s}(0)}{\left(1-a_{i q} t / p_{i q}\right)^{p_{i q}}\left(1-b_{i q} t / q_{i q}\right)^{q_{i q}}}
$$

with $q=u, d$ and $i=1,2$. This ansatz makes no assumption about the size of the strangeness contributions, but if they are neglected, then (16) directly parameterizes the flavor form factors $F_{i}^{u}$ and $F_{i}^{d}$. The sum $a_{i q}+b_{i q}$ is equal to the logarithmic derivative of $F_{i}^{q-s}$ at $t=0$. We note in passing that one has $(1-a t / p)^{-p} \rightarrow \exp (a t)$ for $p \rightarrow \infty$.

At variance with other approaches, we do not impose the asymptotic behavior $F_{i}^{q} \sim 1 / t^{2}$ or $F_{2}^{q} \sim 1 / t^{3}$ that is predicted by dimensional counting. This is in line with the physical assumption behind our GPD fit discussed later, namely that the hard-scattering mechanism that gives rise to dimensional counting behavior is not relevant for the electromagnetic form factors in the $t$ region where there is data. In this spirit, our global fit aims at describing the existing data and at extrapolating them over a limited range, but it has no ambition to describe the form factors in an asymptotic regime of large $t$.

A fit to our default set of form factor data, including the squared neutron charge radius, provides the parameters compiled in table 5 . The corresponding values of $\chi_{\min }^{2}$ are listed in table 6 . With the exception of $q_{1 u}-p_{1 u}$ the differences $q_{i q}-p_{i q}$ of powers were kept fixed in order to obtain a stable $\chi^{2}$ fit, their values have been optimized by varying them in steps of 0.5 and monitoring the change of $\chi_{\min }^{2}$ for the individual form factors, so as to achieve a uniformly good description of all observables as much as possible.

In terms of $\chi^{2}$ the fit is very good as table 6 reveals, with the minimal $\chi^{2}$ being always smaller than the number of data points. Plots comparing the fit with data are shown in section 6.3. With a total of 16 parameters and the ansatz (16) we can thus obtain an excellent description of all the form factor data we have selected. The low $\chi^{2}$ of our fit does not imply that we have over-parameterized the data: as explained earlier, systematic uncertainties are included in the errors on the form factor data, so that their statistical point-to-point fluctuations are not as large as suggested by the errors. We note that a simpler fit with all $q_{i q}-p_{i q}$ set to zero give still a rather good description of the data, with a global $\chi_{\min }^{2}=182.9$. It does, 


\begin{tabular}{ccccc}
\hline & $a_{i q}+b_{i q}$ & $b_{i q}$ & $p_{i q}$ & $q_{i q}-p_{i q}$ \\
\hline$F_{1}^{u-s}$ & $3.116 \pm 0.102$ & $1.122 \pm 0.101$ & $0.347 \pm 0.061$ & $1.527 \pm 0.110$ \\
$F_{1}^{d-s}$ & $3.184 \pm 0.103$ & $1.638 \pm 0.242$ & $0.278 \pm 0.119$ & 3.5 \\
$F_{2}^{u-s}$ & $3.192 \pm 0.060$ & $0.122 \pm 0.034$ & $1.812 \pm 0.110$ & 2.5 \\
$F_{2}^{d-s}$ & $3.478 \pm 0.149$ & $1.649 \pm 0.250$ & $0.296 \pm 0.100$ & 2.5 \\
\hline
\end{tabular}

Table 5: The parameters of the global fit (16) to our default data set. Parameters without quoted errors are kept fixed in the fit.

\begin{tabular}{lcccccccc}
\hline & total & $-G_{M}^{p}-$ & $R^{p}$ & $G_{M}^{n}$ & $R^{n}$ & $G_{E}^{n}$ & $r_{n E}^{2}$ \\
\hline$\chi_{\text {min }}^{2}$ & 122.3 & 28.8 & 1.8 & 52.7 & 20.4 & 15.3 & 3.4 & 0.0 \\
data points & 178 & 48 & 6 & 54 & 36 & 21 & 12 & 1 \\
\hline
\end{tabular}

Table 6: Total and partial values of $\chi_{\min }^{2}$ for the global power law fit specified by (16) and the parameters in table 5 . For $G_{M}^{p}$ the first value is for the data with $-t<10 \mathrm{GeV}^{2}$ and the second value for $-t>10 \mathrm{GeV}^{2}$.

however, systematically overshoot the very precise $G_{M}^{p}$ data for $-t$ below $1 \mathrm{GeV}^{2}$ and correspondingly has a large partial $\chi_{\min }^{2}=73.4$ for the 48 data points of $G_{M}^{p}$ with $-t<10 \mathrm{GeV}^{2}$.

Since we have a complete set of Sachs form factor data only up to $-t=3.41 \mathrm{GeV}^{2}$, the individual flavor form factors of our global power law fit can only be considered reliable up to this $t$ value. On the other extreme, we have data only for $G_{M}^{p}$ in the range $8.5 \mathrm{GeV}^{2}<-t<31.2 \mathrm{GeV}^{2}$. In this range $F_{1}^{u-s}$ as given by our fit contributes more than $70 \%$ to $G_{M}^{p}$ compared with the other flavor form factors. The same is true for the default GPD fit to be discussed in section 6.3. The results of these two fits differ by at most $17 \%$ for all $-t<31.2 \mathrm{GeV}^{2}$. In this sense, we may regard our fit for $F_{1}^{u}$ as reasonably reliable over this $t$ range.

\section{Strangeness}

Although virtual $s \bar{s}$ pairs may not be rare in the proton, the strangeness form factors $F_{1}^{s}(t)$ and $F_{2}^{s}(t)$ are expected to be small, because they describe the difference between the distributions of strange quarks and antiquarks. This is evident from the corresponding sum rules (7) and should not be surprising because the electromagnetic cur- rent probes the local excess of quarks over antiquarks (or of antiquarks over quarks).

Since the proton has no net strangeness, the strange PDFs satisfy

$$
\int_{0}^{1} \mathrm{~d} x[s(x)-\bar{s}(x)]=0
$$

and the strange Dirac form factor is normalized as

$$
F_{1}^{s}(0)=0 .
$$

By contrast, the strange Pauli form factor at $t=$ 0 is equal to the strangeness magnetic moment, $F_{2}^{s}(0)=\mu_{s}$, and can be nonzero.

In recent years there has been an enormous activity to determine the strangeness form factors $G_{M}^{s}$ and $G_{E}^{s}$ from parity violating elastic scattering, see e.g. [56, 57, 58, 59, 60, 61, 62, 63. Despite this effort it is not yet clear whether or not the strange form factors are significantly nonzero, as can be seen in figure 6. Likewise, recent global fits of parton densities [64, 65, do not provide unambiguous evidence for a nonzero difference $s(x)-\bar{s}(x)$. While the MSTW 2008 result 64 for this quantity is compatible with zero within errors, this is not the case for the analysis of NNPDF 2.2 [65]. Lattice QCD results 66, 67, 68, 69, 70, have much smaller er- 

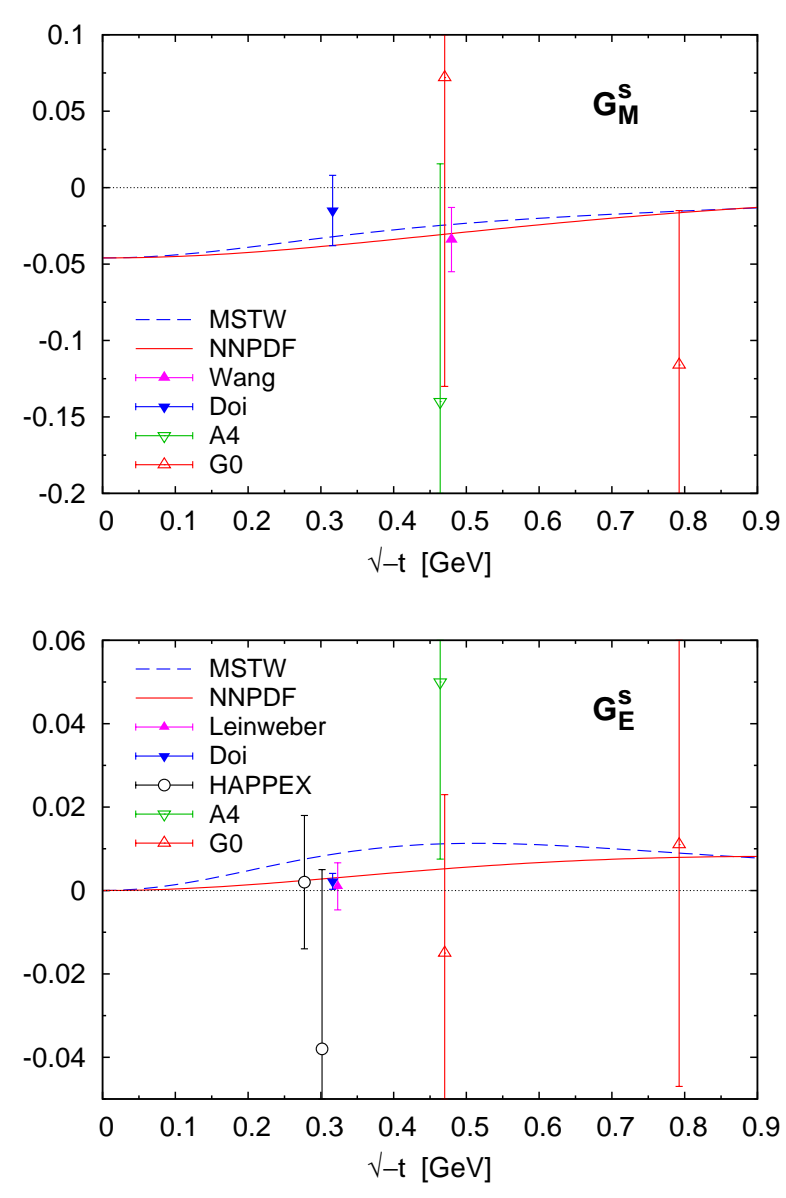

Figure 6: Data for the strangeness form factors by HAPPEX [59, 61], A4 62] and G0 63], together with lattice QCD results by Leinweber [67], Wang [69] and Doi [70. Our model predictions are shown as solid and dashed lines and labeled by the PDF set used in the model.

rors than the form factor measurements and the PDF analyses. Several lattice determinations obtain a nonzero value of $\mu_{s}$ (see below), but $G_{M}^{s}$ and $G_{E}^{s}$ at finite $t$ are typically compatible with zero within uncertainties, as shown in figure 6 .

For an estimate of the strangeness contributions we therefore take recourse to a model. Following our previous work 71 we parameterize the GPD $H_{v}^{s}$ in similar way as the $u$ and $d$ quark GPDs in [5] and in the present paper:

$$
\begin{aligned}
H_{v}^{s}(x, t)= & {[s(x)-\bar{s}(x)] } \\
& \times \exp \left[t \alpha_{s}^{\prime}(1-x) \log (1 / x)\right] .
\end{aligned}
$$

In the exponent we set $\alpha_{s}^{\prime}=0.95 \mathrm{GeV}^{-2}$. Accord- ing to the study in [71, a variation of this value in the range $0.85 \mathrm{GeV}^{-2}<\alpha_{s}^{\prime}<1.15 \mathrm{GeV}^{-2}$ would not significantly change our results for $F_{1}^{s}$. The PDFs in the ansatz (19) are taken either from MSTW 08 64] or from NNPDF 2.2 65] and are evaluated at the scale $\mu=2 \mathrm{GeV}$. With the sum rule (7) one can then compute the Dirac form factor $F_{1}^{s}$. In the region $-t<36 \mathrm{GeV}^{2}$, the result can be parameterized as

$$
F_{1}^{s}(t)=\frac{-c t}{(1-a t / p)^{p}(1-b t / q)^{q}}
$$

with an accuracy better than $2.5 \%$, where $p=2$ and

$$
\begin{aligned}
a & =4.54 \mathrm{GeV}^{-2}, & b & =1.79 \mathrm{GeV}^{-2}, \\
c & =0.136 \mathrm{GeV}^{-2}, & q & =4.8
\end{aligned}
$$

for MSTW, whereas $p=1$ and

$$
\begin{aligned}
a & =2.22 \mathrm{GeV}^{-2}, & b & =0.722 \mathrm{GeV}^{-2}, \\
c & =0.0244 \mathrm{GeV}^{-2}, & q & =1.92
\end{aligned}
$$

for NNPDF.

For the strange Pauli form factor a similar parameterization cannot be exploited, because the forward limit of the relevant GPD is completely unknown. We must therefore pursue a different strategy in this case. Lattice simulations quote the following values for the strangeness magnetic moment:

$$
\begin{aligned}
& \mu_{s}=-0.046 \pm 0.019 \quad \text { [66] } \\
& =-0.066 \pm 0.026 \quad 68 \text {, } \\
& =-0.017 \pm 0.025 \pm 0.007 \quad \text { [70], }
\end{aligned}
$$

and we adopt the value $\mu_{s}=-0.046$ from [66], which is consistent with the other two determinations. There are also estimates of $\mu_{s}$ from different variants of the constituent quark model. Results obtained before 2000 are typically an order of magnitude larger than the lattice results (23), whereas more recent calculations, e.g. in [72, 73, 74, provide results in fair agreement with them.

For the $t$ dependence of $F_{2}^{s}$, we adopt a vector meson dominance ansatz. With three poles corresponding to the $\phi(1020)$ meson and its excited 


\begin{tabular}{lcccccc}
\hline \multicolumn{1}{c}{ reference } & $\begin{array}{c}-t \\
{\left[\mathrm{GeV}^{2}\right]}\end{array}$ & quantity & experimental & \multicolumn{2}{c}{ model result } \\
& & & value & MSTW & NNPDF \\
\hline HAPPEX & {$[57]$} & 0.477 & $G_{E}^{s}+0.392 G_{M}^{s}$ & $0.014 \pm 0.022$ & 0.0032 & -0.00056 \\
A4 & {$[58$} & 0.108 & $G_{E}^{s}+0.106 G_{M}^{s}$ & $0.071 \pm 0.036$ & 0.0057 & -0.00078 \\
HAPPEX & {$[60$} & 0.099 & $G_{E}^{s}+0.080 G_{M}^{s}$ & $0.030 \pm 0.028$ & 0.0061 & $-7 \times 10^{-5}$ \\
HAPPEX & {$[61]$} & 0.109 & $G_{E}^{s}+0.090 G_{M}^{s}$ & $0.007 \pm 0.013$ & 0.0063 & -0.00016 \\
\hline
\end{tabular}

Table 7: Experimental values and model results for linear combinations of strangeness form factors.

states $\phi(1680)$ and $\phi(2170)$ [36] we have ${ }^{1}$

$$
F_{2}^{s}(t)=\mu_{s} \sum_{i=1}^{3} \frac{a_{i}}{m_{i}^{2}-t} .
$$

For lack of better knowledge, we require $F_{2}^{s}$ to decrease asymptotically like $1 / t^{3}$ as suggested by dimensional counting. Together with the normalization condition at $t=0$, this gives the constraints

$$
\begin{aligned}
& \sum_{i} a_{i} / m_{i}^{2}=1, \quad \sum_{i} a_{i}=0, \\
& \sum_{i} a_{i} \sum_{j \neq i} m_{j}^{2}=0,
\end{aligned}
$$

which imply

$$
a_{i}=\frac{m_{1}^{2} m_{2}^{2} m_{3}^{2}}{\prod_{j \neq i}\left(m_{i}^{2}-m_{j}^{2}\right)}
$$

for the residues. With the mass values from [36] we obtain $a_{1}=2.115 \mathrm{GeV}^{2}, a_{2}=-4.113 \mathrm{GeV}^{2}$ and $a_{3}=1.998 \mathrm{GeV}^{2}$. The first parameter is related to the tensor coupling between the $\phi(1020)$ and the nucleon as

$$
g_{\phi N N}^{T}=\mu_{s} f_{1} a_{1} / m_{1}^{2} .
$$

With $f_{1}=13.4$ from the electronic decay width of the $\phi(1020)$ meson, we obtain

$$
\left(g_{\phi N N}^{T}\right)^{2} /(4 \pi)=0.13,
$$

\footnotetext{
${ }^{1}$ We emphasize that $\phi(1020)$ and its excited states are necessary to obtain a proper large $t$ behavior of the strangeness form factors. This sheds doubt on analyses that obtain the dipole behavior of isosinglet form factors $\left(G_{M}^{p}+G_{M}^{n}, G_{E}^{p}+G_{E}^{n}\right.$ or $\left.F_{1}^{p}+F_{1}^{n}\right)$ by a conspiracy of $\omega$ and $\phi$ exchange without excited states. See also our discussion in section 3.1 of 71 .
}

which is consistent with a dispersion analysis of nucleon-nucleon scattering [75]. We note in passing that for $-t<5 \mathrm{GeV}^{2}$ our parameterization of $F_{2}^{s}$ can be approximated by a dipole form (14) with $M_{\text {dip }}^{2}=1.13 \mathrm{GeV}^{2}$ with $11 \%$ accuracy.

Combining our models for $F_{1}^{s}$ and $F_{2}^{s}$ we obtain the Sachs form factors shown in figure 6 . Both models are consistent with experiment and with lattice results, except for a discrepancy between the MSTW model and the lattice points of Leinweber and Doi at $-t=0.1 \mathrm{GeV}^{2}$. Furthermore, our model results are in good agreement with the data listed in table 7, except for the A4 measurement, where our values are about 2 standard deviations away from the experimental value. We note that the combination of the lattice results [70] for $G_{M}^{s}$ and $G_{E}^{s}$ at $-t=0.1 \mathrm{GeV}^{2}$ gives $G_{E}^{s}+0.106 G_{M}^{s}=0.0006 \pm 0.0031$ (if we add errors in quadrature), which is in better agreement with our model results and in tension with the $\mathrm{A} 4$ value.

Comparing our strangeness form factors with the power-law fit of $F_{i}^{u-s}$ and $F_{i}^{d-s}$ described in section 3.4, we find that the ratios $\left|F_{i}^{s} / F_{i}^{u}\right|$ and $\left|F_{i}^{s} / F_{i}^{d}\right|$ are below $6 \%$ for $\sqrt{-t}<1 \mathrm{GeV}^{2}$ and below $12 \%$ for $\sqrt{-t}<2 \mathrm{GeV}^{2}$. With the interpolated set of flavor form factors described in the next section, we find that our strangeness form factors are at most of the size of the errors on $F_{i}^{u}$ and $F_{i}^{d}$. In this respect the strangeness contribution may be neglected when discussing the flavor decomposition of the form factors, at the present level of accuracy. We will return to the strangeness form factors in section 6.4 


\section{$5 \quad$ Interpolated data}

\subsection{Determination of flavor form fac- tors}

The valence quark GPDs $H_{v}^{q}$ and $E_{v}^{q}$ are constrained by the flavor form factors through the sum rules (7). In order to exploit all available information we will directly fit the GPDs to the data on the Sachs form factors. Nevertheless, we find it useful to extract also the experimental values of the flavor form factors, which may be regarded as the form factor set that is most suitable for an interpretation in terms of quark and antiquark densities. This extraction will allow us to verify whether the different GPDs obtained in a global fit satisfy the sum rules (7) with uniform quality. It can also be used to test simple functional forms for the flavor form factors. This may be of use for lattice QCD studies, which typically require a parameterization of simulation results for the extrapolation to the physical values of parameters. Finally, form factor fits are not guaranteed to reproduce local structures in the data, because the flexibility of the assumed parameterization might be insufficient for the structure in question. By contrast, the directly extracted flavor form factors retain local structures present in the data.

For the determination of the flavor form factors, the four Sachs form factors are needed at the same set of $t$ values. This is in general not the case for the available data, since each measurement has its own criteria for a suitable choice of bins in $t$. We therefore need an interpolation procedure. As basic set of $t$ values we choose those of the electric neutron form factor $G_{E}^{n}$ and of the associated form factor ratio $R^{n}$. Compared to the other form factors or ratios, these observables are measured at the smallest number of $t$ values. In this way we avoid having more interpolated data points for the other observables than are actually measured. We omit a few data points for $G_{E}^{n}$, because there are $R^{n}$ measurements at exactly or approximately the same value of $t$. We thus obtain a basic set of 27 values of $-t$ between 0.039 and $3.41 \mathrm{GeV}^{2}$. For these values we interpolate $G_{M}^{p}, G_{M}^{n}$ and $R^{p}$ using cubic splines.

In figure 7 the resulting interpolated data points

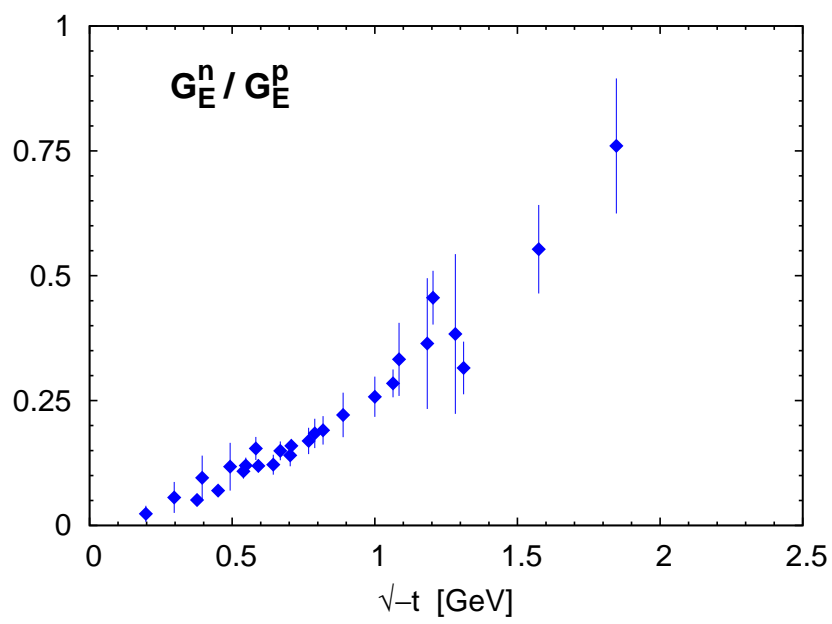

Figure 8: The ratio $G_{E}^{n} / G_{E}^{p}$, evaluated with our set of interpolated data.

are compared to the measured ones. The data on the magnetic from factors of proton and neutron are well represented by the interpolated set. For the ratio $R^{p}$ of electric and magnetic proton form factors, we again see the tensions between different measurements for $\sqrt{-t}$ between 0.6 and $1.0 \mathrm{GeV}$. Following our discussion in section 3 . we use the precise recent Hall A data [17, 18, 19 for interpolating $R^{p}$, as well as the older Hall $\mathrm{A}$ measurement [14] for the high- $t$ region. We finally check the compatibility between the original data on $G_{E}^{n}$ and on $R^{n}$, which are both part of the interpolated form factor set. To this end we take our interpolated values of $G_{M}^{n}$ and compute $R^{n}$ when $G_{E}^{n}$ is measured and vice versa. As can be seen in figure 7, there is good agreement within the uncertainties.

An interesting observation can be made from the set of interpolated data. We see in figure 8 that $G_{E}^{n} \ll G_{E}^{p}$ at low $t$, as one may expect, whereas with increasing $\sqrt{-t}$ the ratio $G_{E}^{n} / G_{E}^{p}$ increases and becomes of order 1 as $\sqrt{-t}$ approaches $2 \mathrm{GeV}$. This finding may be taken as a hint at a zero crossing in the isovector combination $G_{E}^{p}-G_{E}^{n}$ of electric form factors.

From the interpolated Sachs form factors we compute the flavor form factors using (3) and (5), where the strangeness contributions are ne- 

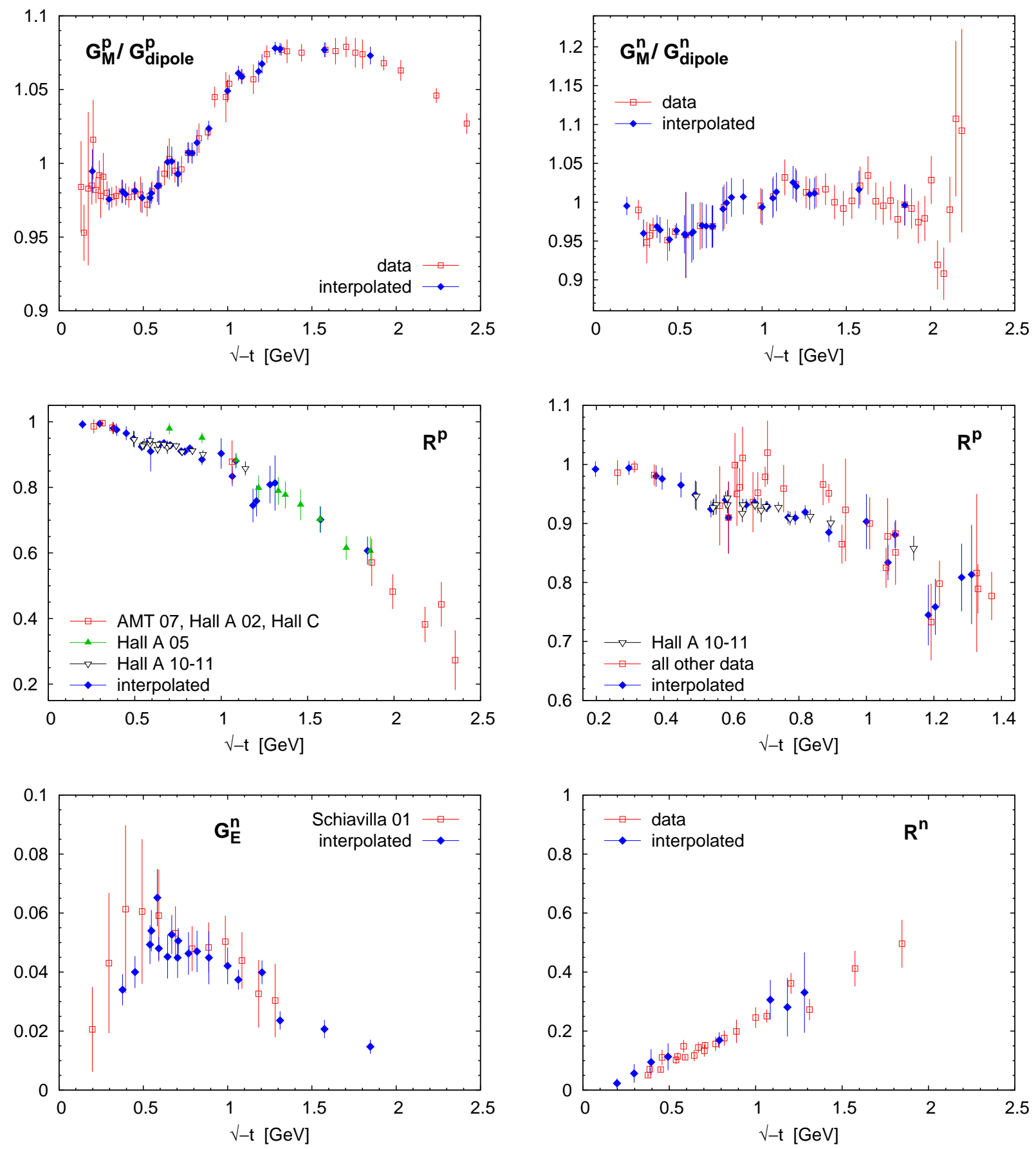

Figure 7: Comparison of interpolated (solid diamonds) and measured (open symbols) Sachs form factors and form factor ratios. For $R^{p}$ the Hall A data of 2005 [14] and of 2010-11 [17, 18, 19] have been used in the interpolation. References for the other data are given in section 3 . 
glected $1^{2}$ The errors of the flavor form factors are evaluated from those of the uncorrelated Sachs form factors or form factor ratios with the help of Gaussian error propagation 3 In the same manner we can compute any combination of the flavor form factors, including its errors. Our results for $F_{i}^{u}$ and $F_{i}^{d}$ are compiled in appendix $\mathrm{A}$ and shown in figure 9. One can see that $F_{2}^{d}$ is negative while the other three form factors are positive, and that

$$
F_{1}^{d}<F_{1}^{u}, \quad F_{2}^{d} \simeq-F_{2}^{u} .
$$

These properties reflect the normalization of the form factors at $t=0$,

$$
\begin{array}{ll}
F_{1}^{u}(0)=2, & F_{1}^{d}(0)=1, \\
F_{2}^{u}(0)=\kappa_{u}, & F_{2}^{d}(0)=\kappa_{d},
\end{array}
$$

where $\kappa_{q}$ is the contribution of quarks with flavor $q$ to the anomalous magnetic moment of the proton $\left(\kappa_{u}=1.67\right.$ and $\kappa_{d}=-2.03$ if strangeness is neglected). One may also notice that with increasing $-t$ the ratio $F_{1}^{d} / F_{1}^{u}$ decreases, while $F_{2}^{d} / F_{2}^{u}$ stays rather flat (see figure 14 below). The decrease of $F_{1}^{d} / F_{1}^{u}$ was already visible in the flavor form factors extracted from our earlier work, see [76, 77, and its relation to the large- $x$ behavior of the parton densities was pointed out in $[5]$. We will take up the discussion of the flavor form factors in sections 6.3 and 7.1 .

In figure 9 we also show the flavor form factors obtained with the global power-law fit described in section 3.4, neglecting again the strangeness contributions. Evidently, this fit describes the flavor form factors very well. The results of the fit are also decomposed into the contributions from the individual Sachs form factors to a given flavor form factor. We notice that in the cases of the $d$ quark form factors strong cancellations among the various contributions occur, whereas $F_{1}^{u}$ is dominated by the proton form factors, with the neu-

\footnotetext{
${ }^{2}$ If one does not wish to neglect these contributions, one can simply re-interpret the form factors $F_{i}^{u}$ and $F_{i}^{d}$ of this section as $F_{i}^{u-s}$ and $F_{i}^{d-s}$, as discussed in section 2

${ }^{3}$ Strictly speaking, the values of $R^{p}$ are not independent of the results for $G_{M}^{p}$ in the global analysis 9 , because that analysis used several of the $R^{p}$ measurements contained in our data set. Since we have no possibility to take this correlation into account, we treat the data for $R^{p}$ and $G_{M}^{p}$ as uncorrelated.
}

tron form factors providing only small contributions. These observations tell us that the interpolated $F_{1}^{u}$ data are quite stable against modifications of the Sachs form factors. The $d$-quark form factors, on the other hand, are rather sensitive to modifications of the data. One may also notice that the contribution to $F_{1}^{u}$ from $G_{E}^{n}$, which is only measured up to $-t=3.41 \mathrm{GeV}^{2}$, is very small. Therefore, the power-law fit as well as the GPD fits described in section 6 are still reliable at $-t$ above $3.4 \mathrm{GeV}^{2}$. By contrast, the contributions of $G_{E}^{n}$ to the other flavor form factors, in particular those for $d$-quarks, are rather important and, hence, our fits to these form factors are to be taken with due caution at large $t$.

Recently, two extractions of the flavor form factors have been published [42, 78. The results for $F_{1}^{u}$ and $F_{1}^{d}$ obtained in [42] are rather similar to ours. The same holds for $F_{2}^{u}$, with slightly larger differences. For $-F_{2}^{d}$, however, the values in [42] are systematically larger than ours by up to $10 \%$ for $-t$ around $1 \mathrm{GeV}^{2}$ and smaller by a similar amount for $-t$ around $3.2 \mathrm{GeV}^{2}$. To understand this discrepancy, we note that [42] uses a fit for $G_{M}^{n}$ that includes the new Hall B data 23] but also the older data sets Anklin 98 and Kubon 02 [20, 21 that we partially discard for the reasons discussed in section 3.2. As can be seen in figure 9 , the impact of $G_{M}^{n}$ is largest on the $d$-quark form factors; for $F_{1}^{d}$ the difference between our values and those of [42] is less visible because the overall errors on it are larger than for $F_{2}^{d}$.

Comparing our flavor form factors with those extracted in [78, we find again rather similar values for $F_{1}^{u}$ and $F_{1}^{d}$. For $F_{2}^{u}$, however, the points of [78] are below ours by up to $15 \%$ for $-t$ around $1 \mathrm{GeV}^{2}$, whereas for $-F_{2}^{d}$ they are above ours by a similar amount in the same $t$ range. These discrepancies are significant at the scale of the quoted errors in the two analyses. Their main origin is that [78] uses the Kelly parameterization [50] for $G_{M}^{n}$ and for the proton form factors. Apart from being in conflict with the Hall B data on $G_{M}^{n}$, this parameterization closely follows the older data on $R^{p}$ and thus lies significantly above the recent Hall A results [17, 18, 19] for $-t$ below $1 \mathrm{GeV}^{2}$. In [78] only the errors of $G_{E}^{n}$ and $R^{n}$ are taken into account while the uncertainties of the Kelly pa- 

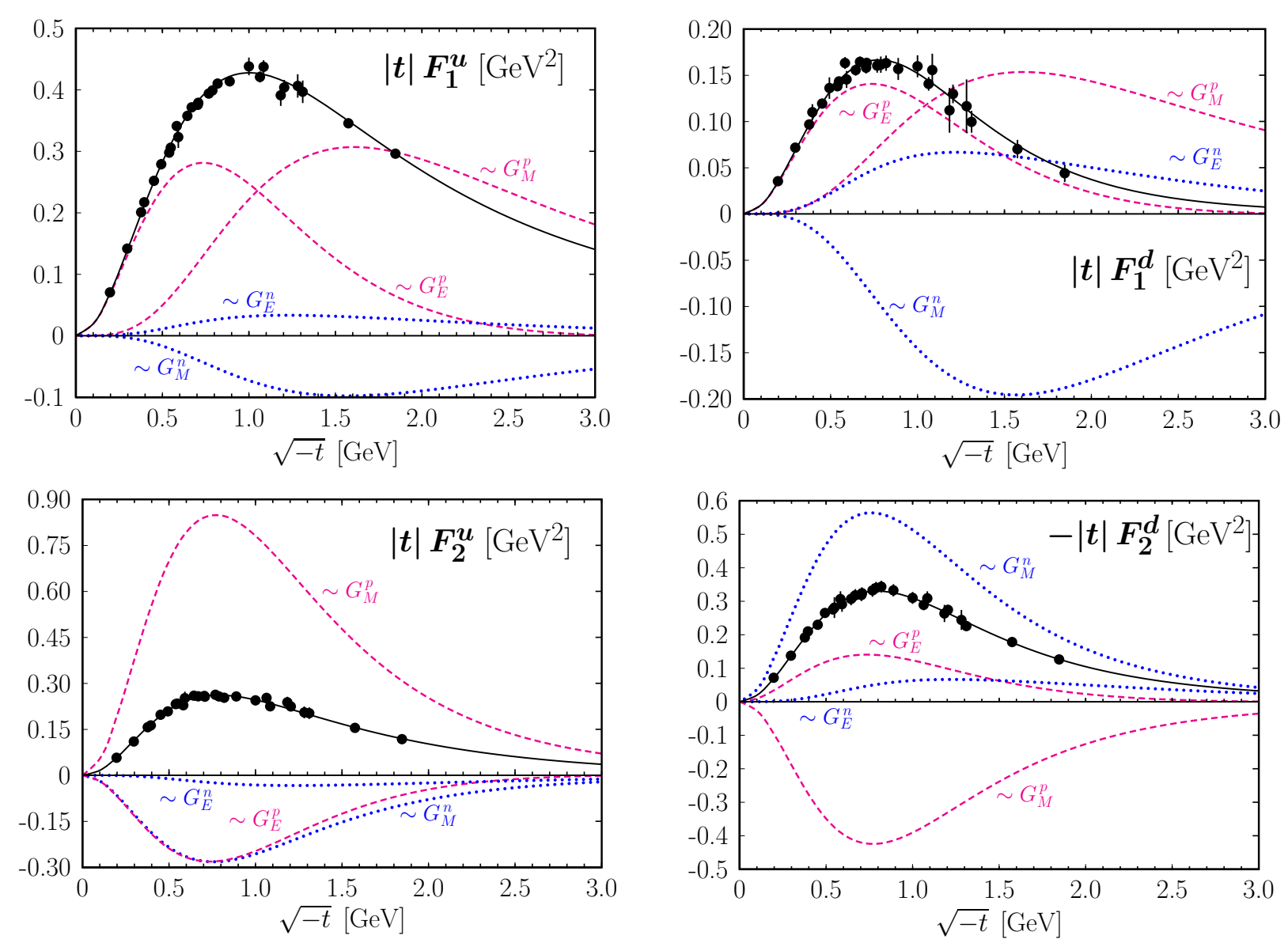

Figure 9: The flavor combinations of the Dirac form factors, scaled by $|t|$ and plotted versus $\sqrt{-t}$. The individual contributions from the proton (neutron) Sachs form factors, computed from the powerlaw fit of section 3.4, are shown as magenta dashed (blue dotted) lines. The solid lines represent the sum of the individual contributions.

rameterization of the other Sachs form factors are ignored. Therefore the errors of the flavor form factors quoted in [78] are smaller (for $F_{2}^{u}$ and $F_{2}^{d}$ even substantially smaller) than ours. We finally stress that, in contrast with [42] and [78], we use only data points and no parameterizations to construct our interpolated data set.

\subsection{Simple fits to form factors}

Lattice QCD studies often require simple parameterizations of form factors for the purpose of interpolation and extrapolation. A representation like the one used in our global power-law fit (16) can normally not be used for this purpose, be- cause it involves 4 parameters per form factor. We can use our interpolated data set to investigate which functional forms are suitable to describe the electromagnetic form factors. In addition to the dipole form (14), we will consider the general power law

$$
F(t)=\frac{F(0)}{\left(1-t / M_{p}^{2}\right)^{p}}
$$

and as a special case also a tripole form, i.e. (31) with $p=3$. As another extension of the dipole parameterization we consider the product of two single poles,

$$
F(t)=\frac{F(0)}{\left(1-t / M_{a}^{2}\right)\left(1-t / M_{b}^{2}\right)} .
$$




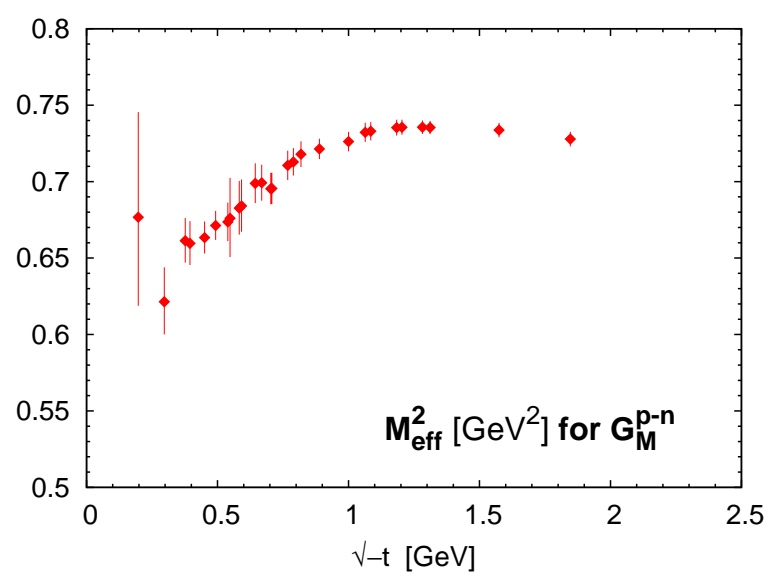

Figure 10: Effective dipole mass (15) for the isovector combination $G_{M}^{p}-G_{M}^{n}$ of magnetic form factors.

A summary of our fits is given in table 8. For each form factor we fit 27 data points in the range $0.039 \mathrm{GeV}^{2} \leq-t \leq 3.41 \mathrm{GeV}^{2}$.

Let us first discuss the Sachs form factors in the isospin basis, i.e. the combinations $G_{M}^{u \pm d}$ and $G_{E}^{u \pm d}$. They form the most natural basis from the point of view of $t$-channel exchanges and of analytic continuation to positive $t$, as is e.g. discussed in section 4.2 of [2]. A dipole or a product of two poles may hence appear as a natural candidate to describe these form factors. We find that a dipole form describes our interpolated values for $G_{M}^{u-d}$ with an accuracy better than $5 \%$. On the scale of the errors on $G_{M}^{u-d}$, this is however a poor description, and the associated $\chi_{\min }^{2}$ is very high as we see in table 8 . That $G_{M}^{u-d}$ cannot be described by a dipole within its uncertainties is confirmed by a plot of the corresponding effective dipole mass (see section 3.3) in figure 10. A product of two poles gives a better description, but still with a $\chi_{\min }^{2}$ almost twice as big as the number of data points.

The isosinglet combinations $G_{M}^{u+d}$ and $G_{E}^{u+d}$ have larger error bars than $G_{M}^{u-d}$, and we find that they can be described reasonably well by a dipole. In both cases, a product of two poles, which has one more free fit parameter, gives an even better description. As for $G_{E}^{u-d}$, we find that a dipole form gives a reasonable description up to $\sqrt{-t}=1.3 \mathrm{GeV}$ but badly fails to reproduce the two data points with $\sqrt{-t}=1.57 \mathrm{GeV}$ and $1.85 \mathrm{GeV}$, as is reflected in the accuracy and $\chi_{\min }^{2}$ given in table 8 . This is not too surprising if we recall that this form factor may have a zero around $\sqrt{-t}=2 \mathrm{GeV}$, as we observed when commenting on figure 8. A two-parameter fit of $G_{E}^{u-d}$ to the product of two poles is unstable.

Turning now to the Dirac and Pauli form factors in the isospin basis, we find that $F_{1}^{u+d}$ is reasonably well described by a dipole, and even better by the product of two poles. By contrast, a dipole form is unable to describe $F_{1}^{u-d}$. The product of two poles permits a description with $6 \%$ accuracy, which has however still a large $\chi_{\min }^{2}$. A general power-law fit is slightly worse for this form factor, which has very small errors. The isotripolet Pauli form factor $F_{2}^{u-d}$ is also known with high precision. We find a very poor description by a dipole fit, and an even worse one by a tripole form, whose asymptotic $t$ behavior corresponds to the dimensional counting prediction for $F_{2}^{u-d}$ (and is obviously irrelevant for the $t$ range in question). A general power law does better in comparison, with an accuracy of about $6 \%$ but still a bad $\chi_{\min }^{2}$. The isoscalar combination $F_{2}^{u+d}$ has rather large errors and is equally well described by a dipole and a tripole fit. A similar situation has often been found in lattice studies, when the errors on the simulation did not permit to draw strong conclusions on the $t$ dependence of certain form factors.

Turning finally to the flavor basis, we observe that a dipole fit gives a rather poor description for $F_{1}^{u}, F_{1}^{d}$ and $F_{2}^{d}$. The general power law (31) works however very well in all three cases. It works also very well for $F_{2}^{u}$, where the fitted power is close to 2 , so that a dipole fit is adequate in this exceptional case.

In conclusion, we have not found a simple "one fits all" functional form that would describe either the Sachs or the Dirac and Pauli form factors in the isospin basis. The only ansatz that gives a uniformly good description of all form factor data for $-t$ up to $3.4 \mathrm{GeV}^{2}$ is to fit the flavor form factors to the general power law (31) with its two free parameters. 


\begin{tabular}{ccccc}
\hline form factor & \multicolumn{2}{c}{ dipole } & & two poles \\
& accuracy & $\chi_{\min }^{2}$ & accuracy & $\chi_{\min }^{2}$ \\
\hline$G_{M}^{u-d}$ & $-4.5 \%$ to $2.5 \%$ & 197.9 & $-4.5 \%$ to $2.0 \%$ & 49.1 \\
$G_{M}^{u+d}$ & $-8.0 \%$ to $5.5 \%$ & 15.3 & $-4.5 \%$ to $4.0 \%$ & 6.7 \\
$G_{E}^{u+d}$ & $-5 \%$ to $13 \%$ & 20.8 & $-9 \%$ to $9 \%$ & 13.5 \\
$G_{E}^{u-d}$ & $-75 \%$ to $10 \%$ & 63.1 & & \\
\hline
\end{tabular}

\begin{tabular}{ccccc}
\hline form factor & \multicolumn{2}{c}{ dipole } & & \multicolumn{2}{c}{ two poles -} \\
& accuracy & $\chi_{\min }^{2}$ & accuracy & $\chi_{\min }^{2}$ \\
\hline$F_{1}^{u+d}$ & $-5.0 \%$ to $9.5 \%$ & 36.4 & $-5.0 \%$ to $8.0 \%$ & 27.3 \\
$F_{1}^{u-d}$ & $-12 \%$ to $29 \%$ & 946 & $-5.5 \%$ to $6.5 \%$ & 69.2 \\
$F_{2}^{u-d}$ & $-19 \%$ to $4 \%$ & 433 & & \\
$F_{2}^{u+d}$ & $-7.5 \%$ to $5.5 \%$ & 24.2 & & \\
\hline
\end{tabular}

\begin{tabular}{cccccc}
\hline form factor & \multicolumn{2}{c}{ power law } & & & tripole - \\
& accuracy & $\chi_{\min }^{2}$ & accuracy & $\chi_{\min }^{2}$ \\
\hline$F_{1}^{u+d}$ & $-4.5 \%$ to $9.0 \%$ & 30.9 & & \\
$F_{1}^{u-d}$ & $-6 \%$ to $8 \%$ & 105 & & \\
$F_{2}^{u-d}$ & $-6.5 \%$ to $5.5 \%$ & 113 & $-7 \%$ to $28 \%$ & 1037 \\
$F_{2}^{u+d}$ & & & $-6.5 \%$ to $5.5 \%$ & 17.2 \\
\hline
\end{tabular}

\begin{tabular}{cccccc}
\hline form factor & \multicolumn{2}{c}{ dipole } & & \multicolumn{3}{c}{ power law } \\
& accuracy & $\chi_{\min }^{2}$ & accuracy & $\chi_{\min }^{2}$ & $p$ \\
\hline$F_{1}^{u}$ & $-7 \%$ to $14 \%$ & 138 & $-4.0 \%$ to $5.5 \%$ & 17.9 & $1.13 \pm 0.03$ \\
$F_{1}^{d}$ & $-50 \%$ to $10 \%$ & 54.0 & $-19 \%$ to $16 \%$ & 13.0 & $2.81 \pm 0.18$ \\
$F_{2}^{u}$ & $-12.5 \%$ to $8.5 \%$ & 15.0 & $-5.0 \%$ to $10.0 \%$ & 9.6 & $2.16 \pm 0.07$ \\
$F_{2}^{d}$ & $-24 \%$ to $8 \%$ & 30.0 & $-8.5 \%$ to $7.5 \%$ & 11.2 & $2.38 \pm 0.10$ \\
\hline
\end{tabular}

Table 8: Quality of fits to our interpolated form factor data with different simple functional forms. For each form factor, 27 data points are fitted. As "accuracy" we define the smallest and largest value of $(1-\mathrm{fit} /$ data). If a field is left empty, no stable fit of acceptable quality could be found. The column "power law" refers to the form (31) and the column "two poles" to (32). The last column in the last table gives the value of $p$ in the power-law fit. 


\section{GPD fit}

\subsection{Fit ansatz and positivity}

Let us now briefly describe our ansatz for the GPDs, which will be used in our fits to the form factors. We largely follow the approach of our earlier work [5] and refer to it for a more detailed motivation and discussion. The main feature of our ansatz is an exponential $t$ behavior

$$
\begin{aligned}
H_{v}^{q}(x, t) & =q_{v}(x) \exp \left[t f_{q}(x)\right], \\
E_{v}^{q}(x, t) & =e_{v}^{q}(x) \exp \left[t g_{q}(x)\right],
\end{aligned}
$$

with an $x$ dependent width specified by the profile functions $f_{q}(x)$ and $g_{q}(x)$. For polarized quarks we assume

$$
\widetilde{H}_{v}^{q}(x, t)=\Delta q_{v}(x) \exp \left[t f_{q}(x)\right],
$$

where for lack of better knowledge we take the same $t$ dependence as for $H_{v}^{q}$ (see section 8.1). The above forms refer to a definite renormalization scale $\mu$, which we take equal to $2 \mathrm{GeV}$ unless stated otherwise.

An intuitive interpretation of GPDs at zero skewness can be given in impact parameter space, where we define

$$
\begin{aligned}
& q_{v}\left(x, \boldsymbol{b}^{2}\right)=\int \frac{\mathrm{d}^{2} \boldsymbol{\Delta}}{(2 \pi)^{2}} e^{-i \boldsymbol{b} \boldsymbol{\Delta}} H_{v}^{q}\left(x,-\boldsymbol{\Delta}^{2}\right), \\
& e_{v}^{q}\left(x, \boldsymbol{b}^{2}\right)=\int \frac{\mathrm{d}^{2} \boldsymbol{\Delta}}{(2 \pi)^{2}} e^{-i \boldsymbol{b} \boldsymbol{\Delta}} E_{v}^{q}\left(x,-\boldsymbol{\Delta}^{2}\right) .
\end{aligned}
$$

$q_{v}\left(x, \boldsymbol{b}^{2}\right)$ is difference of densities for quarks and antiquarks with momentum fraction $x$ at a transverse distance $\boldsymbol{b}$ from the proton center, with both the parton and the proton being unpolarized. The average impact parameter associated with this density difference is

$$
\left\langle\boldsymbol{b}^{2}\right\rangle_{x}^{q}=\frac{\int \mathrm{d}^{2} \boldsymbol{b} \boldsymbol{b}^{2} q_{v}\left(x, \boldsymbol{b}^{2}\right)}{\int \mathrm{d}^{2} \boldsymbol{b} q_{v}\left(x, \boldsymbol{b}^{2}\right)}=4 f_{q}(x) .
$$

The corresponding density difference for longitudinally polarized partons is

$$
\Delta q_{v}\left(x, \boldsymbol{b}^{2}\right)=\int \frac{\mathrm{d}^{2} \boldsymbol{\Delta}}{(2 \pi)^{2}} e^{-i \boldsymbol{b} \boldsymbol{\Delta}} \widetilde{H}_{v}^{q}\left(x,-\boldsymbol{\Delta}^{2}\right),
$$

whereas for unpolarized partons in a proton polarized along the $x$-axis one has [79]

$$
q_{v}^{X}(x, \boldsymbol{b})=q_{v}\left(x, \boldsymbol{b}^{2}\right)-\frac{b^{y}}{m} \frac{\partial}{\partial \boldsymbol{b}^{2}} e_{v}^{q}\left(x, \boldsymbol{b}^{2}\right) .
$$

Transverse polarization of the proton thus induces a sideways shift in the distribution of partons. The average amount of this shift is

$$
\left\langle b^{y}\right\rangle_{x}^{q}=\frac{\int \mathrm{d}^{2} \boldsymbol{b} b^{y} q_{v}^{X}\left(x, \boldsymbol{b}^{2}\right)}{\int \mathrm{d}^{2} \boldsymbol{b} q_{v}^{X}\left(x, \boldsymbol{b}^{2}\right)}=\frac{1}{2 m} \frac{e_{v}^{q}(x)}{q_{v}(x)} .
$$

The interpretation as a density difference requires $q_{v}^{X}(x, \boldsymbol{b}) \geq 0$ when the antiquark contribution is negligible. This implies a bound on $\partial /\left(\partial \boldsymbol{b}^{2}\right) e_{v}^{q}\left(x, \boldsymbol{b}^{2}\right)$, which becomes even stronger if we include information about polarized quarks. In a region where antiquarks can be neglected, we then have 81 .

$$
\begin{aligned}
\frac{\boldsymbol{b}^{2}}{m^{2}}\left[\frac{\partial}{\partial \boldsymbol{b}^{2}}\right. & \left.e_{v}^{q}\left(x, \boldsymbol{b}^{2}\right)\right]^{2} \\
& \leq\left\{\left[q\left(x, \boldsymbol{b}^{2}\right)\right]^{2}-\left[\Delta q\left(x, \boldsymbol{b}^{2}\right)\right]^{2}\right\} .
\end{aligned}
$$

With (33) and (34), the validity of (40) for all $\boldsymbol{b}$ at a given $x$ is equivalent to 5

$$
\begin{gathered}
\frac{\left[e_{v}^{q}(x)\right]^{2}}{8 m^{2}} \leq \exp (1)\left[\frac{g_{q}(x)}{f_{q}(x)}\right]^{3}\left[f_{q}(x)-g_{q}(x)\right] \\
\times\left\{\left[q_{v}(x)\right]^{2}-\left[\Delta q_{v}(x)\right]^{2}\right\} .
\end{gathered}
$$

Note that 41) requires strict inequality $g_{q}(x)<$ $f_{q}(x)$ of the profile functions, except for values of $x$ where $e_{v}^{q}(x)=0$. As we will see, the positivity bound on $e_{v}^{q}(x)$ severely constrains our fits.

As a word of caution, we note that the density interpretation and the associated positivity conditions do not strictly hold in QCD. This is because the ultraviolet renormalization that makes the GPDs well defined and leads to their $\mu$ dependence involves subtractions that can in principle invalidate positivity. We nevertheless require the above conditions to hold, so that a density interpretation is possible for the results of our fits. The technical implementation of the positivity conditions is discussed in the next subsection.

We now specify our ansatz (33) and (34). For $q_{v}(x)$ we take a selection of up-to-date parton densities, which is discussed in section 6.2, and for 
the polarized densities $\Delta q_{v}(x)$ we choose the recent determination [80. All these distributions are defined with NLO evolution and evaluated at scale $\mu=2 \mathrm{GeV}$. For $e_{v}^{q}(x)$, we make the ansatz

$$
e_{v}^{q}(x)=\kappa_{q} N_{q} x^{-\alpha_{q}}(1-x)^{\beta_{q}}\left(1+\gamma_{q} \sqrt{x}\right),
$$

which has proven to work well for the parameterization of ordinary parton densities. The normalization factor $N_{q}$ ensures that

$$
\int_{0}^{1} \mathrm{~d} x e_{v}^{q}(x)=\kappa_{q}
$$

as required by (7). The values of $\kappa_{u}$ and $\kappa_{d}$ are computed from the measured magnetic moments of proton and neutron and the assumed value for the strangeness contribution $\kappa_{s}=\mu_{s}$. The $\gamma_{q}$ dependent term in (42) is new compared with [5] and significantly improves our fits, as we will discuss in sections 6.3 and 6.4 .

For the profile functions $f_{q}(x)$ and $g_{q}(x)$ we assume the form 5 ]

$$
\begin{aligned}
f_{q}(x)= & \alpha_{q}^{\prime}(1-x)^{3} \log (1 / x)+B_{q}(1-x)^{3} \\
& +A_{q} x(1-x)^{2} \\
g_{q}(x)= & \alpha_{q}^{\prime}(1-x)^{3} \log (1 / x)+D_{q}(1-x)^{3} \\
& +C_{q} x(1-x)^{2} .
\end{aligned}
$$

The parameters $\alpha_{q}^{\prime}, B_{q}$ and $D_{q}$ control the small- $x$ behavior of these functions, whereas their behavior at large $x$ is controlled by $A_{q}$ and $C_{q}$. The factors of $(1-x)$ in $f_{q}(x)$ ensure that $\left\langle\boldsymbol{b}^{2}\right\rangle_{x}^{q} \sim(1-x)^{2}$ in the limit $x \rightarrow 1$, which follows from requiring a finite transverse size of the proton in that limit (see section 7.3).

At small $x$, the $\log (1 / x)$ term in $g_{q}(x)$ gives a $t$-dependent contribution to the power behavior $E_{v}^{q}(x, t) \sim x^{-\left(\alpha_{q}+t \alpha_{q}^{\prime}\right)}$, in accordance with simple Regge phenomenology. A corresponding statement holds for $H_{v}^{q}(x, t)$ if the forward densities $q_{v}(x)$ have a power behavior at small $x$.

\subsection{Selection of parton densities}

An important feature of our ansatz (33) is that for the forward limit of $H_{v}^{q}(x, t)$ we can use the valence quark densities obtained in global PDF analyses. As we shall see, current PDF determinations exhibit notable differences for the valence quark densities $u_{v}(x)$ and $d_{v}(x)$, especially in the regions of small or large $x$. Since these regions are of some importance in the sum rules (7) (see section 7.1), we have explored several recent PDF determinations in our fits. They are all defined at NLO, evaluated at $\mu=2 \mathrm{GeV}$, and listed in table 9. The numerical values for all parton densities have been obtained with the routines of the LHAPDF interface [82, version 5.8.8. From now on we will denote the PDF sets only by the acronyms of their authors (ABM, CT, etc.) since we only use one set from each group.

As already mentioned in the previous subsection, the power behavior at small $x$, which is suggested by simple Regge phenomenology, is an important ingredient of the physical motivation for our GPD ansatz. We shall see in section 7.1 that the power behavior of the GPDs at large $x$ is closely related to the large- $t$ behavior of the form factors. We have therefore taken a closer look at the behavior of the parton densities at small and at large $x$. To quantify this behavior, we fit the PDFs to effective power laws

$$
\begin{array}{rlrl}
q_{v}(x) & \sim x^{-\alpha_{q}^{\mathrm{eff}}} \quad & \text { for } 10^{-3}<x<10^{-2} \\
& \sim(1-x)^{\beta_{q}^{\mathrm{eff}}} \quad \text { for } 0.65<x<0.85 .
\end{array}
$$

The effective powers we obtain are given in table 9. In all cases the accuracy of the fit is better than 5\%. Our choice of $x$ intervals in the fits comes from the requirements that they should be of importance in the sum rules (7) and that the PDFs should indeed follow an approximate power law behavior. If we fit the small- $x$ behavior for $10^{-4}<x<10^{-3}$ then the effective powers decrease by 0.0 to 0.02 , with the following exceptions: for CT $\alpha_{d}^{\mathrm{eff}}$ decreases by 0.04, for NNPDF $\alpha_{d}^{\text {eff }}$ decreases by 0.05 , and for MSTW $\alpha_{d}^{\text {eff }}$ decreases by 0.08 and $\alpha_{u}^{\text {eff }}$ by 0.10

As it is evident from the effective powers in table 9, there is a significant variation between different PDF sets, and we must conclude that neither the small- $x$ nor the large- $x$ behavior of the valence quark distributions is presently known with certainty. This is also seen in the plots of the different PDFs in figure 11 . We further observe that the spread between different PDF sets 


\begin{tabular}{|c|c|c|c|c|c|}
\hline $\mathrm{PDF}$ & ref. & $\alpha_{u}^{\mathrm{eff}}$ & $\alpha_{d}^{\mathrm{eff}}$ & $\beta_{u}^{\mathrm{eff}}$ & $\beta_{d}^{\mathrm{eff}}$ \\
\hline $\mathrm{ABM} 11 n_{f}=4$ & 83 & 0.33 & 0.34 & 3.5 & 5.0 \\
\hline CT 10 & 84 & 0.39 & 0.42 & 3.4 & $\begin{array}{ll}3.6 & 2.7\end{array}$ \\
\hline GJR $08 \mathrm{VF}$ & 85 & 0.54 & 0.53 & 3.8 & 4.9 \\
\hline HERAPDF 1.5 & 86 & 0.33 & 0.33 & 4.2 & 4.8 \\
\hline MSTW 2008 & 64 & 0.54 & 0.29 & 3.5 & 5.9 \\
\hline NNPDF 2.2 & 65 & 0.43 & 0.28 & 3.5 & 4.5 \\
\hline
\end{tabular}

Table 9: The PDF sets used in our analysis and the effective powers for their behavior at small and large $x$ as defined in (45). All PDFs are evaluated at scale $\mu=2 \mathrm{GeV}$. The two values of $\beta_{d}^{\text {eff }}$ for the CT 10 set correspond to separate fits in the ranges $0.65<x<0.75$ and $0.75<x<0.85$.

is larger than the error bands of the individual PDFs, which is not surprising since the latter reflect parametric errors of the PDF fits but not systematic uncertainties of the fitting procedure. Rather than the errors on a given PDF set, we will hence use the variation from different sets in order to estimate the uncertainty induced on our analysis of GPDs and form factors.

Returning to table 9, we observe that in the MSTW and NNPDF sets there is a significant difference in the effective powers for $u$ and $d$ quarks at small $x$, which we consider to be in tension with usual Regge phenomenology. At large $x$, we find that the $d$ quark distribution of the CT analysis has a rather peculiar behavior: it does not follow an approximate power behavior over a significant range in $x$, and for $x \gtrsim 0.8$ it is significantly larger than in any other PDF set (including the earlier set CTEQ 6M [87] of the same collaboration).

For our default GPD fit we have chosen the ABM set. With the GJR set, our fit gives a value of $\alpha$ in the parameterization of $e_{v}^{q}(x)$ that we consider at the limit of what is plausible from Regge phenomenology, and our fit with the HERAPDF set turns out to have a relatively large $\chi^{2}$ compared with the other PDFs.

Let us note that for extremely large $x$, say above 0.9, some (although not all) PDFs obtained with the LHAPDF interface behave unexpectedly, either by not being monotonic in $x$ or by becoming negative. Since the PDFs are extremely small in that region, this may be due to numerical instabilities, which would explain that the problems occur mostly for $d_{v}(x)$. We have however not investigated this issue further. In any case, the results of global PDF fits for such large $x$ must be regarded as extrapolations, since there is no experimental data constraining them in that region. This is illustrated by the fact that already at $x=0.9$ the PDF sets we have chosen exhibit a spread of almost a factor 4 for $u_{v}(x)$ and an even larger spread for $d_{v}(x)$. Luckily, the uncertainty in this $x$ region does not affect our analysis in a significant way, since such values of $x$ do not dominate the integrals over GPDs that give form factors in the $t$ range where there is data. We will quantify this in section 7.1.

Let us now specify how we implement the conditions from positivity in our GPD fits. We require the validity of (41) for $x>0.15$, since for smaller $x$ antiquarks are found to become important in the forward parton densities. At large $x$, where it is plausible to neglect antiquarks, two types of problem complicate using the positivity bound.

- As described in the previous paragraph, there are numerical instabilities in the parton densities. To stay away from this region we do not enforce the bound (41) for $x>0.9$. We do however require $g_{q}(x)<f_{q}(x)$ in that region, since this bound is independent of the PDFs.

- With the polarized PDFs of [80 and some of the unpolarized PDF sets, the requirement $\left|\Delta q_{v}(x)\right|<q_{v}(x)$ is not satisfied for very large $x$. Specifically, we find $\Delta u_{v}(x)>u_{v}(x)$ for the sets GJR $(x>0.78)$ and HERAPDF 

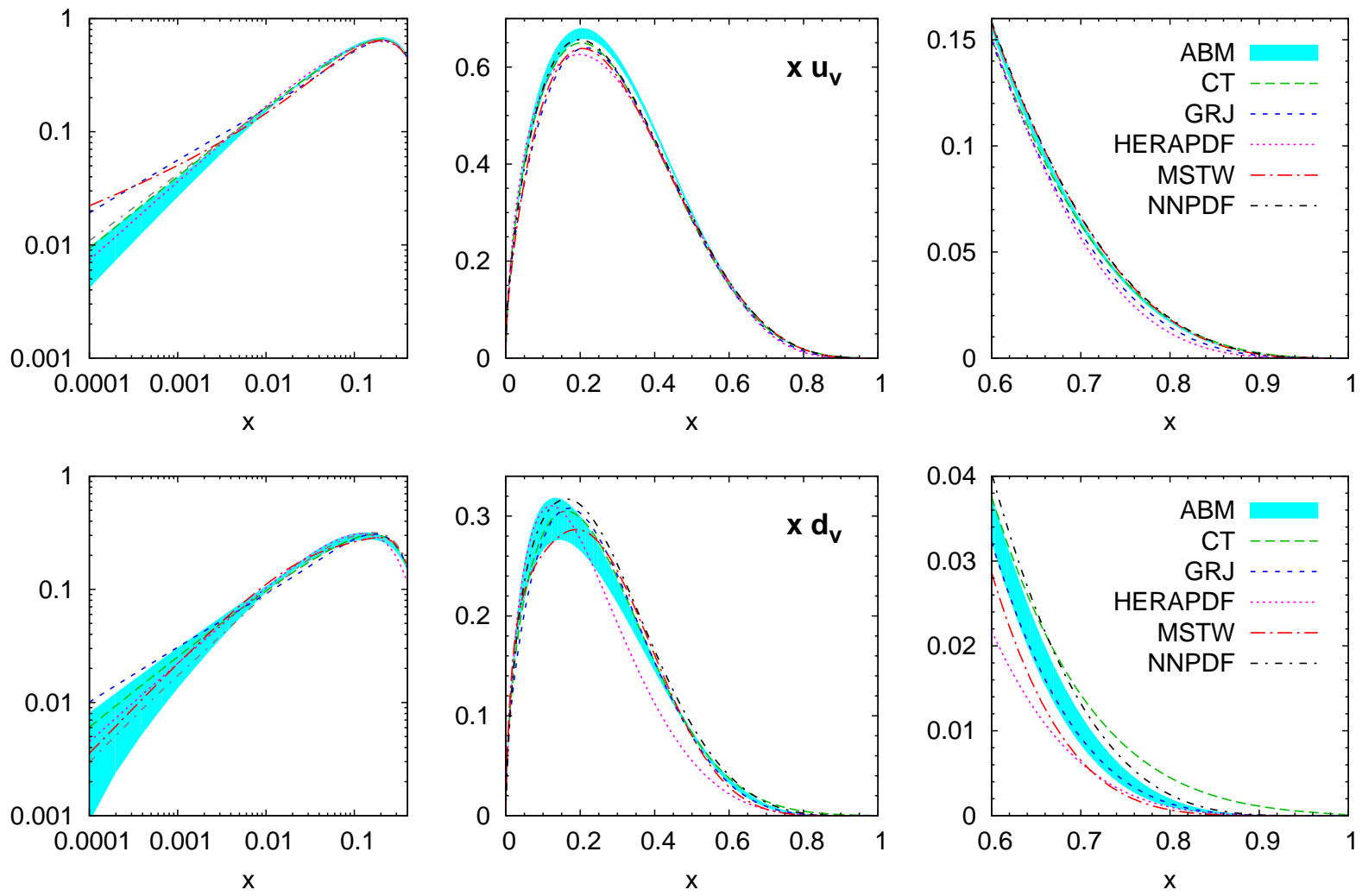

Figure 11: The valence densities of $u$ quarks (top) and $d$ quarks (bottom) for the PDF sets specified in table 9 .

$(x>0.72)$, whereas $-\Delta d_{v}(x)>d_{v}(x)$ for $\operatorname{ABM}(x>0.83)$, GJR $(x>0.82)$, HER$\operatorname{APDF}(x>0.69)$, MSTW $(x>0.71)$ and NNPDF $(x>0.91)$. This is not too surprising, since positivity in conjunction with those unpolarized PDFs was not enforced in the DSSV extraction [80], and it does not represent a physics problem given the overall uncertainties on the parton densities in the relevant $x$ region.

To circumvent this problem at a technical level, we set the polarized PDFs to zero in the bound (41) for those values of $x$ where $\left|\Delta q_{v}(x)\right|>0.9 q_{v}(x)$.

\subsection{The default fit}

We now have all elements needed for our fit of GPDs to the form factor data. In this section we discuss what we consider our best fit, where in particular we set the strangeness form factors
$F_{1}^{s}$ and $F_{2}^{s}$ to zero. Different variants of this fit are presented in the next section. Compared with our analysis [5] we have significantly extended the form factor data used in the fit. This allows for a larger number of free parameters and for a simultaneous fit of $H_{v}^{q}$ and $E_{v}^{q}$ (with $q=u, d$ ) to all data. In [5] we had instead first computed Dirac and Pauli form factors from the experimental results and then performed separate fits of $H_{v}^{q}$ and $E_{v}^{q}$.

Nevertheless, we cannot allow all parameters in (42) and (44) to vary independently. Fits with too many free parameters do not only give very large parameter uncertainties but also tend to violate the positivity constraints. To limit the number of free parameters, we appeal to Regge phenomenology. Assuming that the small- $x$ behavior of both $E_{v}^{q}$ and $H_{v}^{q}$ is dominated by the leading meson trajectories, namely those of the $\rho$ and the $\omega$, and that those trajectories are degenerate, we obtain that to first approximation the small- $x$ 
powers $\left(\alpha_{q}+t \alpha_{q}^{\prime}\right)$ in $E_{v}^{q}$ should be equal for $u$ and $d$ quarks. They should also be equal to the analogous powers in $H_{v}^{q}$, which is why we have taken the same parameters $\alpha_{q}^{\prime}$ in the profile functions $f_{q}$ and $g_{q}$, see (44). The $t$ dependent part of the small- $x$ power for $H_{v}^{q}$ is not a fit parameter but a property of the PDFs in our ansatz, and for the ABM parameterization we take in our default fit the effective powers $\alpha_{\text {eff }}^{q}$ are nearly equal for $u$ and $d$ quarks.

We emphasize that the equality of the small- $x$ powers in $E_{v}^{u}, E_{v}^{d}, H_{v}^{u}$ and $H_{v}^{d}$ is not expected to be exact. The meson trajectories are not exactly degenerate and Regge phenomenology allows for subleading trajectories and Regge cuts, which can all lead to different effective powers when the GPDs are approximated by a single power law in a certain $x$ range. Moreover, one cannot expect to find the literal values of meson trajectories in the small- $x$ behavior of GPDs or PDFs. Indeed, parton distributions are subject to scale evolution, which changes the effective $x$ powers, although rather slowly as long as a single power law gives a good description over a large interval in $x$.

Our approach is thus to take equal small- $x$ powers as long as our fit does not require otherwise. We thus set

$$
\alpha_{u}=\alpha_{d}=\alpha
$$

for the small- $x$ powers in the forward functions $e_{v}^{u}$ and $e_{v}^{d}$, given that we do not find significantly better fits if we allow $\alpha_{u}$ and $\alpha_{d}$ to differ.

On the other hand we find that, together with the abundant and precise low $t$ data on several form factors, the very precise value (13) of the squared neutron charge radius $r_{n E}^{2}$ requires some deviation from full degeneracy of the small- $x$ powers. With $\alpha_{u}^{\prime}=\alpha_{d}^{\prime}$ we obtain poor partial $\chi^{2}$ values for $r_{n E}^{2}$ and for the $R^{n}$ data. A good description can however be obtained with a slight isospin breaking of the form $\alpha_{u}^{\prime}>\alpha_{d}^{\prime}$. We therefore take

$$
\alpha_{u}^{\prime}-\alpha_{d}^{\prime}=0.1 \mathrm{GeV}^{-2}
$$

in our fits. A further increase of $\alpha_{u}^{\prime}-\alpha_{d}^{\prime}$ yields an even better $\chi^{2}$, but we do not consider a large isospin splitting to be physically motivated.

Let us now discuss the parameters $\gamma_{u}$ and $\gamma_{d}$ in the forward functions $e_{v}^{u}(x)$ and $e_{v}^{d}(x)$, which are new compared with our study [5]. We have varied these parameters independently in steps of 1 and selected the values

$$
\gamma_{u}=4, \quad \gamma_{d}=0
$$

for our default fit. Compared with setting both $\gamma_{u}$ and $\gamma_{d}$ to zero, this decreases the overall $\chi^{2}$ by about 30 units, with most significant improvements for $G_{M}^{p}$ and $G_{M}^{n}$. Taking $\gamma_{u}$ even larger improves the $\chi^{2}$ only slightly and leads to larger fitted values of $\alpha$. We thus retain (48) as a compromise between a good $\chi^{2}$ and parameters in line with Regge phenomenology. If we take either $\gamma_{u}$ or $\gamma_{d}$ as free fit parameters, then they have large errors of order $50 \%$ while $\chi^{2}$ improves only moderately compared with 48.

We observe that all our fits are very significantly influenced by the positivity constraints, in accord with our previous analysis [5]. In particular, we find that if we leave $\beta_{u}$ and $\beta_{d}$ (or one of them) as free parameters, then their fitted values are very low $\left(\beta_{u}=3.5\right.$ and $\beta_{d}=1.6$ if both are left free). This badly violates the positivity bound (41). To circumvent this problem, we perform fits for fixed values of $\beta_{u}$ and $\beta_{d}$ on a grid with step size 0.05 . The resulting values of $\chi^{2}$ are given in table 10 . We see that the minimum of $\chi^{2}$ in the $\left(\beta_{u}, \beta_{d}\right)$ plane occurs at the boundary of the region allowed by our positivity conditions, with values

$$
\beta_{u}=4.65, \quad \beta_{d}=5.25 .
$$

We label this fit as ABM 1 and refer to it as our "default fit" in the remainder of this work. It yields the parameters

$$
\begin{aligned}
\alpha_{d}^{\prime} & =(0.861 \pm 0.026) \mathrm{GeV}^{-2}, \\
\alpha & =0.603 \pm 0.020
\end{aligned}
$$

and the values in table 11. We consider (50) to be consistent with expectations from Regge phenomenology. The value of $\alpha$ is somewhat large compared with the intercepts of the leading meson trajectories, but we deem it still acceptable. We shall further discuss the parameters $\alpha$ and $\beta_{u}$, $\beta_{d}$ in section 6.4 .

The parametric uncertainties in the fit are of reasonable size, with the most precisely determined parameters being $\alpha, \alpha_{d}^{\prime}$ (and hence $\left.\alpha_{u}^{\prime}\right), A_{u}$ 


\begin{tabular}{|c|cccccccccc|}
\hline & & & \multicolumn{7}{|c|}{$\beta_{u}$} \\
& & 4.65 & 4.70 & 4.75 & 4.80 & 4.85 & 4.90 & 4.95 & 5.00 & 5.05 \\
\hline 5.20 & - & - & - & - & - & 221.9 & 222.0 & 222.2 & 222.3 \\
5.25 & 221.2 & 221.4 & 221.6 & 221.7 & 221.9 & 222.0 & 222.2 & 222.3 & 222.4 \\
5.30 & 221.3 & 221.5 & 221.7 & 221.9 & 222.0 & 222.2 & 222.3 & 222.4 & 222.5 \\
5.35 & 221.4 & 221.6 & 221.8 & 222.0 & 222.1 & 222.3 & 222.4 & 222.5 & 222.6 \\
& 5.40 & 221.6 & 221.8 & 221.9 & 222.1 & 222.3 & 222.4 & 222.5 & 222.7 & 222.8 \\
$\beta_{d}$ & 5.45 & 221.7 & 221.9 & 222.1 & $\frac{222.2}{222.4}$ & 222.5 & 222.6 & 222.8 & 222.9 \\
& 5.50 & 221.8 & 222.0 & 222.2 & 222.3 & 222.5 & 222.6 & 222.8 & 222.9 & 223.0 \\
& 5.55 & 221.9 & 222.1 & 222.3 & 222.4 & 222.6 & 222.7 & 222.9 & 223.0 & 223.1 \\
5.60 & 222.0 & 222.2 & 222.4 & 222.6 & 222.7 & 222.8 & 223.0 & 223.1 & 223.2 \\
5.65 & 222.1 & 222.3 & 222.5 & 222.7 & 222.8 & 222.9 & 223.1 & 223.2 & 223.3 \\
5.70 & 222.2 & 222.4 & 222.6 & 222.8 & 222.9 & 223.1 & 223.2 & 223.3 & 223.4 \\
5.75 & 222.3 & 222.5 & 222.7 & 222.9 & 223.0 & 223.1 & 223.3 & 223.4 & 223.5 \\
\hline
\end{tabular}

Table 10: Values of $\chi^{2}$ for fits with the same setting as our default fit (ABM 1). Underlined values indicate the overall minimum and the one-sigma contour. Positivity is violated in the fits with $\beta_{d}=5.2$ and $\beta_{u} \leq 4.85$, as well as for all fits with $\beta_{d} \leq 5.15$ or $\beta_{u} \leq 4.6$.

\begin{tabular}{ccr}
\hline$q$ & $u$ & \multicolumn{1}{c}{$d$} \\
\hline$A_{q}$ & $1.264 \pm 0.050$ & $4.198 \pm 0.231$ \\
$B_{q}$ & $0.545 \pm 0.062$ & $0.206 \pm 0.073$ \\
$C_{q}$ & $1.187 \pm 0.087$ & $3.106 \pm 0.249$ \\
$D_{q}$ & $0.333 \pm 0.065$ & $-0.635 \pm 0.076$ \\
\hline
\end{tabular}

Table 11: Parameters of the profile functions $f_{q}$ and $g_{q}$ (see (44)) in our default fit. All quantities have the unit $\mathrm{GeV}^{-2}$.

and $A_{d}$. In general there are strong correlations between all parameters. We can obtain an uncertainty estimate on $\beta_{u}$ and $\beta_{d}$ using the criterion $\Delta \chi^{2}=1$. This gives an asymmetric contour in the $\left(\beta_{u}, \beta_{d}\right)$ plane, which is marked in table 13 . We see that the large- $x$ powers of $e_{v}^{q}$ are determined with reasonable although not very high precision.

When computing GPDs we use standard linear error propagation for the free parameters in the fit; the necessary matrix is given in appendix $B$. We use a simplified procedure to propagate the errors on $\beta_{u}$ and $\beta_{d}$ into GPDs and observables that are derived from them. Namely, we compute the quantity in question for each of the 7 fits that have $\Delta \chi^{2}=1$ w.r.t. the default fit in table 10 and compare the result with the value obtained with the default fit. If the difference is larger than what is obtained with standard error propagation for the free parameters in the default fit, we retain it for the error estimate. A more elaborate procedure would also scan the fits with $\Delta \chi^{2}<1$ in the $\left(\beta_{u}, \beta_{d}\right)$ plane, but we refrain from doing so for the sake of simplicity. We find that the uncertainties due to the variation of $\beta_{q}$ are not important for the electromagnetic form factors, whose error is therefore given by standard error propagation for the free fit parameters. The variation of $\beta_{q}$ is however relevant for the second and third $x$-moments of GPDs (including the angular momentum sum rule), for the shift $s_{q}(x)$ to be discussed in section 7.3 and for the model estimate of the Sivers distributions in section 8.3. In those cases, changes in $e_{v}^{q}(x)$ due to the variation of $\beta_{q}$ are not compensated by changes in the profile functions $g_{q}(x)$, in constrast to what happens for the electromagnetic form factors to which the GPDs are fitted.

We note that our default fit corresponds to a local but not the global minimum of $\chi^{2}$ in the 
$\left(\beta_{u}, \beta_{d}\right)$ plane. The global minimum is also assumed at the boundary of the $\beta_{q}$ values allowed by positivity, namely at the largest possible $\beta_{u}$. We find it at $\beta_{u}=18.85$ and $\beta_{d}=5.25$. It has $\chi^{2}=209.8$, which is about 11 units smaller than in our default fit. At the global minimum we find that $g_{u}(x)$ is nearly zero at $x \sim 0.3$. Both because of this and because of the very high value of $\beta_{u}$, we do not consider this fit to be physically plausible and retain the local minimum of $\chi^{2}$ at the low end of the allowed $\beta_{q}$ values instead.

Let us now see how well our default fit describes the data. Its overall $\chi^{2}$ is 221.2 for 178 data points. Partial $\chi^{2}$ values are given in table 13 below, and plots in figure 12. For the sake of discussion we split the $G_{M}^{p}$ data into a low- $t$ and a high- $t$ sample, with their boundary being at $-t=10 \mathrm{GeV}^{2}$. We find that the fit provides a very good description of the neutron form factors, i.e. of $G_{M}^{n}, G_{E}^{n}$ and $R^{n}$, and also of $G_{M}^{p}$ at large $t$. The description is still fair but less optimal for $G_{M}^{p}$ at low $t$ and for $R^{p}$, with partial $\chi^{2}$ values of about 1.7 and 1.5 per data point, respectively. As shown in figure 12 the fit slightly overshoots the very precise $G_{M}^{p}$ data for $\sqrt{-t}<0.7 \mathrm{GeV}$, and for $R^{p}$ it fails to reproduce the fine details of the data with $\sqrt{-t}<1 \mathrm{GeV}$. We find the same two shortcomings in all of our alternative GPD fits to the same data set, as we will see in section 6.4. It is not impossible to describe these data more precisely, as is demonstrated by the power-law fit of section 3.4 which achieves partial $\chi^{2}$ values of 0.6 and 1 per data point for $G_{M}^{p}$ at low $t$ and for $R^{p}$, respectively. We conclude that our GPD fits, with the significant constraints in parameter space imposed by positivity, reach their limits of precision here, and we must postpone a resolution of this shortcoming to the future. We note that the low- $t$ data for $R^{p}$ are still subject to experimental debate, as discussed in section 3.1 .

In figure 13 we compare our default GPD fit (as well as our power-law fit) with our interpolated data set for the Dirac and Pauli form factors, both in the quark flavor basis and in the isospin basis. Good agreement can be observed in all cases. We note that the isosinglet Pauli form factor $F_{2}^{u+d}$ is very small due to a strong cancellation between $u$ and $d$ quarks and therefore has
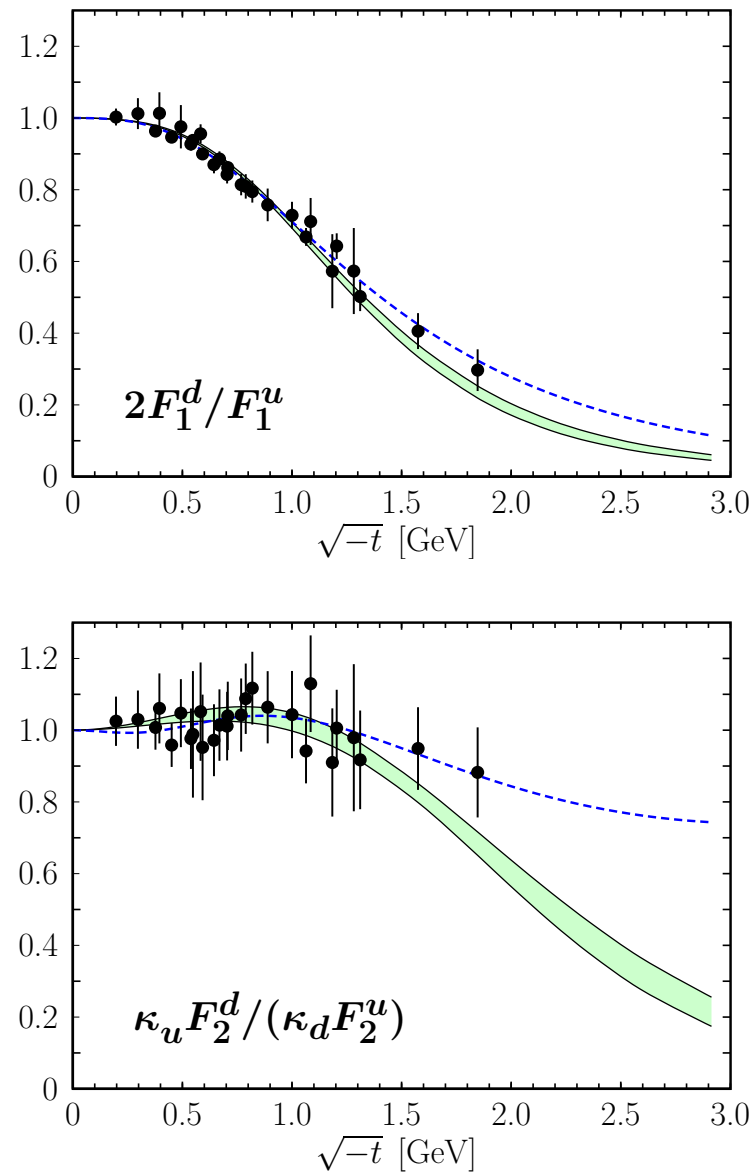

Figure 14: Results of our fits for the ratios of the Dirac and Pauli form factors for $d$ and $u$ quarks, normalized to unity at $t=0$. Data and curves are as in figures 12 and 13 .

large relative errors. Our interpolated data set suggests that it may have a zero crossing at $\sqrt{-t}$ around $2 \mathrm{GeV}$, but more precise data is needed for a definite conclusion.

In figure 14 we finally show the ratios of Dirac and Pauli form factors for $u$ and $d$ quarks. As already mentioned in section 5.1, we have a strong decrease of this ratio for the Dirac form factors, whereas for the Pauli form factors it stays nearly flat. In our default GPD fit (but not in the power-law fit) we obtain a decrease of $\left|F_{2}^{d} / F_{2}^{u}\right|$ for $\sqrt{-t}>1.5 \mathrm{GeV}$, which is not suggested by the data but consistent within their errors. It will be interesting to see the behavior of this ratio with data for larger $-t$. 

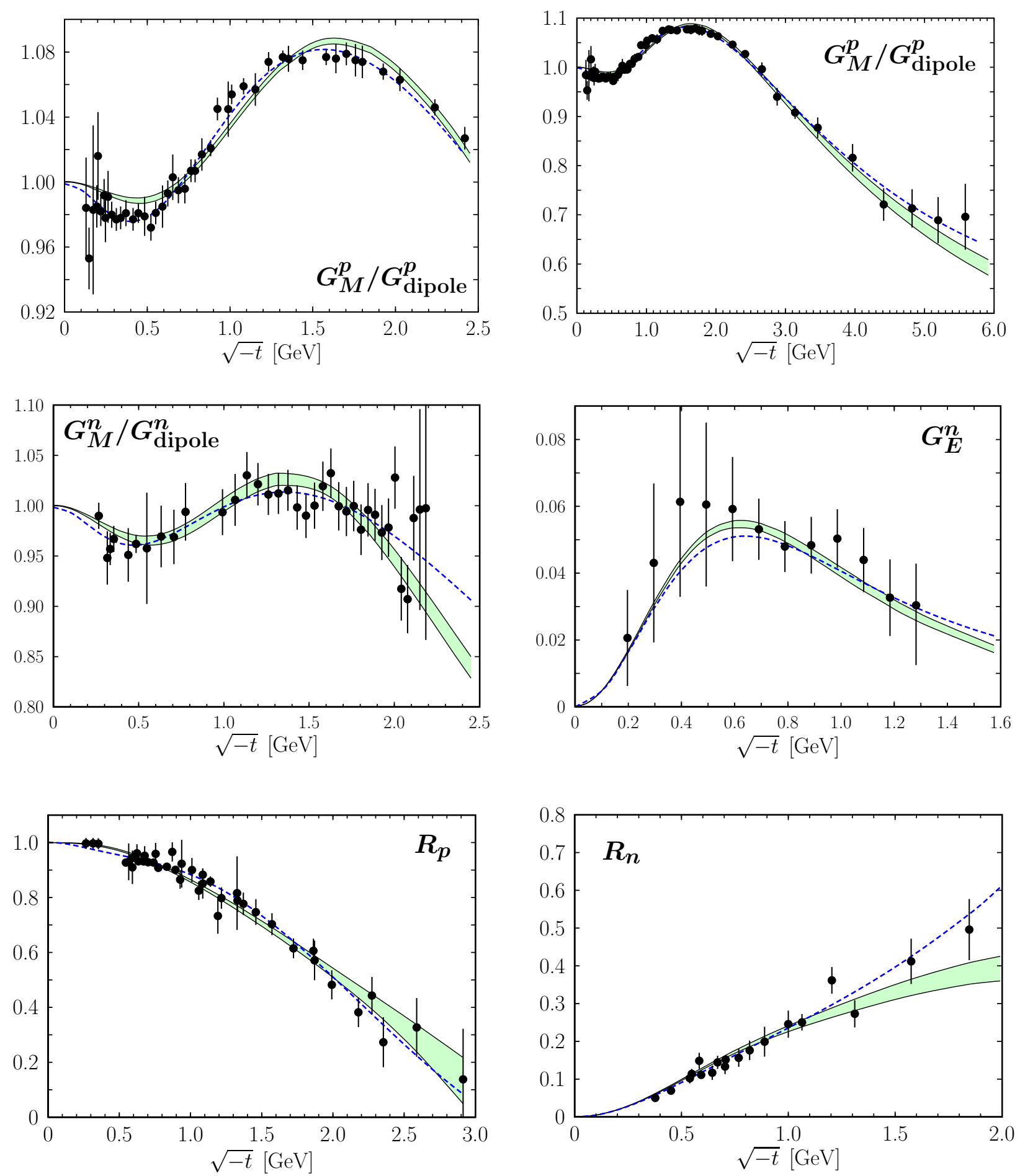

Figure 12: Results of our fits for the Sachs form factors or their ratios. The dashed lines show the power-law fit described in section 3.4 and the bands show the default GPD fit described in the present section. The data points correspond to the default data set specified in table 1 . 

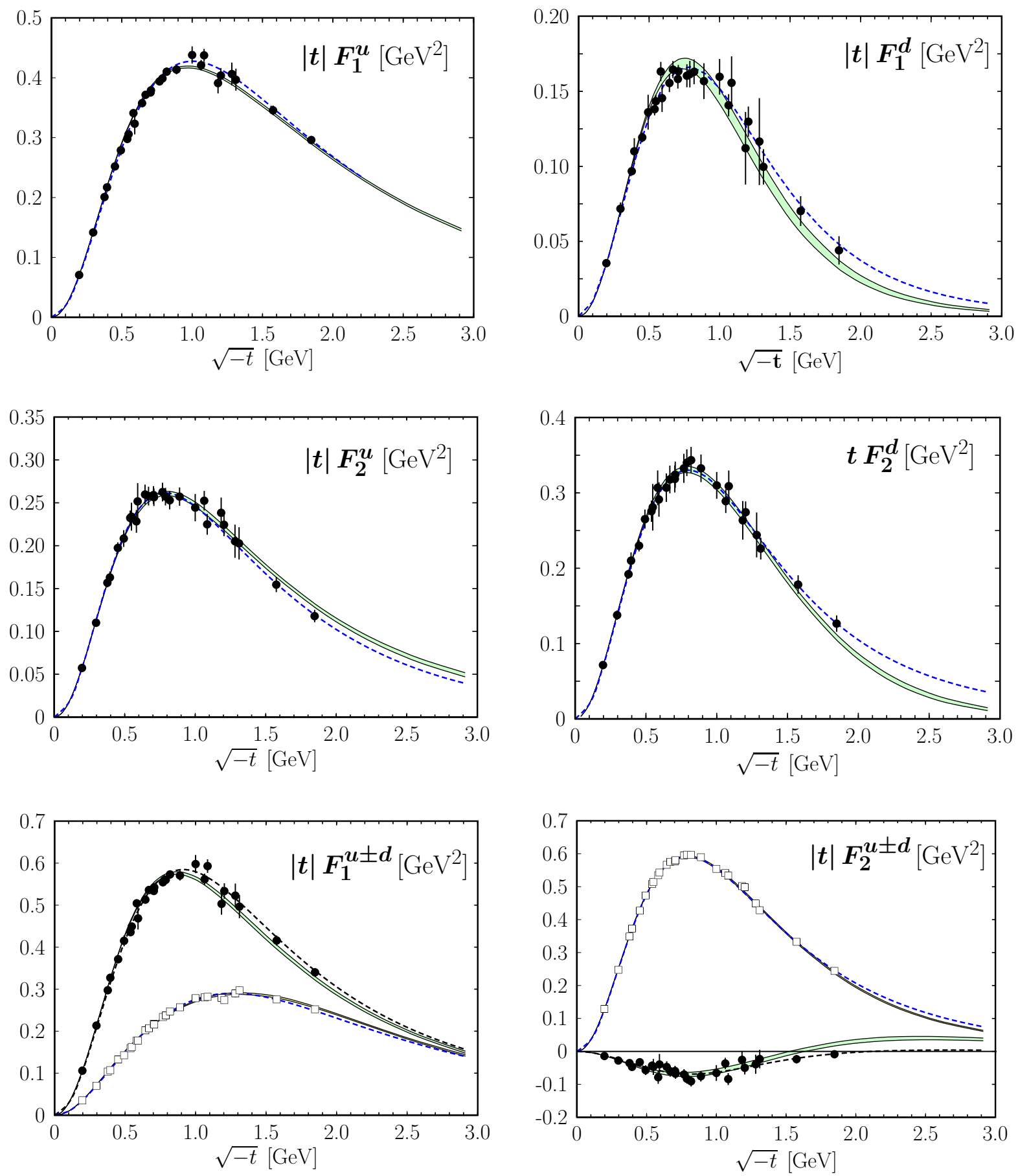

Figure 13: Results of our fits for the flavor form factors, which are obtained by interpolation as described in section 5.1. All form factors are scaled with $|t|$. The dashed lines show the power-law fit described in section 3.4 and the bands show for the default GPD fit. The data for the isotriplet combinations $F_{1}^{u-d}$ and $F_{2}^{u-d}$ are shown as open squares. 


\subsection{Variations of the default fit}

We have performed a number of alternative fits to explore the dependence of our results on several choices we have made in the fitting procedure. A brief description of the different alternative fits is given in table 12, and the corresponding values of $\chi^{2}$ can be found in table 13 . For all fits described in the following, we find again that a local minimum of $\chi^{2}$ in the $\left(\beta_{u}, \beta_{d}\right)$ plane is taken at the boundary of values allowed by the positivity conditions, as was the case for our default fit.

Let us first discuss the alternative fits using the same PDFs as the default fit. We remark that a fit with $\gamma_{u}=\gamma_{d}=0$ has severe problems with positivity. Specifically, it tends to have $A_{u}<C_{u}$ and thus to violate the condition $g_{q}(x)<f_{q}(x)$, except for rather large values $\beta_{u} \geq 6.20$ and $\beta_{d} \geq$ 5.95. Given this and the significantly larger $\chi^{2}$ already mentioned in the previous subsection, we conclude that the data clearly prefer a nonzero $\gamma_{u}$ and do not consider this parameter setting any further.

We now discuss the other fits in turn, referring to their labels in table 12 .

ABM 2. In this fit we set $\gamma_{d}=4$, i.e. equal to $\gamma_{u}$. The description of the data is globally as good as for the default fit ABM 1, with some improvement for $G_{M}^{p}$ and a somewhat worse $\chi^{2}$ for $R^{p}$. The fitted value $\alpha=0.654 \pm 0.017$ is relatively high, which is why we prefer the fit $\mathrm{ABM} 1$ (where $\alpha=0.603 \pm 0.020$ ) as our default.

ABM 3 and 4. In these fits we include the models for the strangeness form factors described in section 4, taking either the strangeness PDFs of MSTW or of NNPDF (all other parton density sets we consider have $s(x)=\bar{s}(x)$ and hence cannot be used to model $\left.F_{1}^{s}\right)$. The global description of the data is just slightly worse than for fit ABM 1, where strangeness is neglected, with a slight preference for the variant using the NNPDF densities in the model for $F_{1}^{s}$. An exception is the poor description of the neutron charge radius in the case of ABM 3. We note that we get a more satisfactory result with $\alpha_{u}^{\prime}-\alpha_{d}^{\prime}=0.2 \mathrm{GeV}^{-2}$ in this case.

Overall, we conclude that the influence of the small strangeness form factors in our fit is visible in the details but does not change the overall picture significantly. This is in line with our findings discussed at the end of section 4 .

ABM 5. In this fit we omit the recent Hall A data for $R^{p}$, namely the sets Paolone 10 , Ron 11 and Zhan 11, and instead include the polarized target data of Jones 06 and Crawford 07 (see table 2). As can be seen in figure 2, this removes the tension between the recent Hall A data and earlier JLab measurements that is present in our default data set. The fit ABM 5 describes the alternative data set for $R^{p}$ very well, with a $\chi^{2}$ of less than 1 per data point. It also gives a significantly improved (although not perfect) description of $G_{M}^{p}$ at low $t$. Only for the neutron charge radius does the description become notably worse compared with fit ABM 1. The data on $R^{p}$ thus has a clear impact on our fits, which confirms the urgency for experimental clarification of the tensions between the current data sets.

ABM 6. To investigate the impact of the $G_{M}^{p}$ data on our fit, we take as an alternative data set the results of Arrington 05 [39], which covers the range $0.141 \mathrm{GeV}^{2} \leq-t \leq$ $9.121 \mathrm{GeV}^{2}$. To have data at higher $-t$, we include the results of our default set, AMT 07 [9], for $-t \geq 9.848 \mathrm{GeV}^{2}$. The Arrington 05 extraction has significantly larger errors than the one of AMT 07 for the reasons discussed in section 3.1, and we correspondingly find a very small $\chi^{2}$ of 0.4 per data point for the low- $t$ data on $G_{M}^{p}$. The description of the other data sets is not much changed compared with fit ABM 1, except for a slight improvement for $R^{p}$.

The dependence of $\chi^{2}$ on the choice of $\left(\beta_{u}, \beta_{d}\right)$ is much less pronounced in fit ABM 6 than in all other fits, so that those parameters are less well determined. Clearly, the high precision of the data on $G_{M}^{p}$ we adopted 


\begin{tabular}{lccl}
\hline fit & $\beta_{u}$ & $\beta_{d}$ & remarks \\
\hline ABM 1 & 4.65 & 5.25 & default fit (see section 6.3) \\
ABM 2 & 5.05 & 5.80 & $\gamma_{d}=\gamma_{u}=4$ \\
ABM 3 & 4.55 & 5.20 & with strangeness (MSTW) \\
ABM 4 & 4.60 & 5.25 & with strangeness (NNPDF) \\
ABM 5 & 4.40 & 5.00 & alternative data for $R^{p}$ \\
ABM 6 & 4.95 & 5.50 & alternative data for $G_{M}^{p}$ \\
\hline ABM 0 & 4.35 & 5.10 & as ABM 1 but at scale $\mu=1 \mathrm{GeV}$ \\
\hline CT & 4.60 & 4.15 & \\
GJR & 4.65 & 5.10 & \\
HERAPDF & 4.70 & 5.35 & \\
MSTW & 4.65 & 5.90 & $\alpha_{u}^{\prime}-\alpha_{d}^{\prime}=0$ \\
NNPDF & 4.70 & 5.15 & \\
\hline
\end{tabular}

Table 12: Overview of our GPD fits. All fits have $\gamma_{u}=4, \gamma_{d}=0, \alpha_{u}^{\prime}-\alpha_{d}^{\prime}=0.1 \mathrm{GeV}^{-2}$ and PDFs evaluated at $\mu=2 \mathrm{GeV}$ unless stated otherwise.

\begin{tabular}{|c|c|c|c|c|c|c|c|c|}
\hline & total & $-G_{N}^{p}$ & $4-$ & $R^{p}$ & $G_{M}^{n}$ & $R^{n}$ & $G_{E}^{n}$ & $r_{n E}^{2}$ \\
\hline data points & 178 & 48 & 6 & 54 & 36 & 21 & 12 & 1 \\
\hline ABM 1 & 221.2 & 79.7 & 3.8 & 78.8 & 29.5 & 24.1 & 3.2 & 2.1 \\
\hline ABM 2 & 219.2 & 71.1 & 4.4 & 85.9 & 27.7 & 25.0 & 3.2 & 1.9 \\
\hline ABM 3 & 230.5 & 81.5 & 2.9 & 76.7 & 29.7 & 31.1 & 3.2 & 5.5 \\
\hline $\mathrm{ABM} 4$ & 225.0 & 79.2 & 3.9 & 81.1 & 29.0 & 26.1 & 3.0 & 2.7 \\
\hline $\mathrm{ABM} 5^{a}$ & 166.4 & 64.5 & 1.9 & 40.8 & 30.5 & 22.0 & 2.9 & 3.7 \\
\hline $\mathrm{ABM} 6^{b}$ & 139.3 & 14.9 & 4.0 & 63.8 & 27.2 & 23.8 & 3.1 & 2.4 \\
\hline ABM 0 & 198.1 & 77.1 & 2.3 & 65.6 & 27.1 & 20.9 & 3.1 & 1.9 \\
\hline $\mathrm{CT}$ & 212.6 & 75.7 & 2.9 & 75.6 & 28.3 & 24.5 & 3.2 & 1.4 \\
\hline GJR & 189.7 & 64.3 & 3.5 & 70.9 & 27.0 & 19.6 & 3.3 & 1.1 \\
\hline HERAPDF & 254.5 & 83.4 & 10.6 & 96.4 & 33.3 & 25.7 & 3.3 & 1.7 \\
\hline MSTW & 167.9 & 53.1 & 2.9 & 63.1 & 24.4 & 17.9 & 4.9 & 1.6 \\
\hline NNPDF & 196.6 & 72.6 & 3.9 & 73.2 & 27.5 & 15.4 & 4.0 & 0.0 \\
\hline power law & 122.3 & 28.8 & 1.8 & 52.7 & 20.4 & 15.3 & 3.4 & 0.0 \\
\hline
\end{tabular}

Table 13: Partial and total values of $\chi^{2}$ for the GPD fits and for the power-law fit of section 3.4 . The first value for $G_{M}^{p}$ refers to the data with $-t<10 \mathrm{GeV}^{2}$ and the second value to the data with $-t>10 \mathrm{GeV}^{2}$. 
in our default fit is of great importance for a precise determination of the GPDs.

ABM 0. In this fit we take the PDFs from the $\mathrm{ABM}$ set, but evaluate them at scale $\mu=$ $1 \mathrm{GeV}$ instead of $2 \mathrm{GeV}$. The electromagnetic form factors are scale invariant and can be described by the sum rules (7) with GPDs at any scale, but of course it does matter at which scale we make the functional ansatz for GPDs specified in section 6.1.

Compared with fit ABM 1, the description of the data in fit ABM 0 is improved significantly for $G_{M}^{p}$ at low $t$ and for $R^{p}$, with little change for the other observables. Since the usual PDFs at $\mu=1 \mathrm{GeV}$ are determined only indirectly (data corresponding to such a low scale is not included in the PDF analyses in order to limit the importance of higher-twist corrections), we prefer the fits with $\mu=2 \mathrm{GeV}$ as our default.

We have also repeated the fit with the other PDF sets discussed in section 6.2, neglecting the strangeness form factors and fixing $\gamma_{u}=4, \gamma_{d}=0$ as in our default fit.

For all PDFs we find that $\alpha_{u}^{\prime}-\alpha_{d}^{\prime}=0.1 \mathrm{GeV}^{-2}$ clearly improves the description of $r_{n E}^{2}$ and of $R^{n}$ compared with $\alpha_{u}^{\prime}-\alpha_{d}^{\prime}=0$. An exception is the fit with the PDFs of MSTW, where this parameter setting makes the fit slightly worse, so that we take $\alpha_{u}^{\prime}=\alpha_{d}^{\prime}$ in this case. This exception is not implausible, since the MSTW set already has a large isospin breaking between $\alpha_{u}^{\text {eff }}$ and $\alpha_{d}^{\text {eff }}$ (see table 9p. A further isospin breaking in the profile functions of $H_{v}^{q}$ and $E_{v}^{q}$ is then visibly not preferred by the fit.

Whereas the fit with the HERAPDF densities describes the form factor data less well than the fit ABM 1, the variants using CT, GJR, MSTW or NNPDF have lower values of $\chi^{2}$. Like in the default fit, the description is least optimal for $G_{M}^{p}$ at low $t$ and for $R^{p}$, whereas the other observables are described very well (except for the fit using HERAPDF, which undershoots the $G_{M}^{p}$ data at high $t$ ). As spelled out in section 6.2, we choose the fit using the ABM set as our default, where the behavior of both $q_{v}(x)$ and $e_{v}^{q}(x)$ at small and at large $x$ corresponds best to the physical picture underlying our parameterization of the GPDs.

The parameters of the profile functions obtained in our GPD fits are shown in figure 15 (the results for $\alpha$ can be found in figure 20 below). Clearly, the parametric uncertainties on $f_{q}(x)$ and $g_{q}(x)$ in a given fit are smaller than their variation due to choosing different PDFs and to other choices made in the fit. This also illustrates the strong correlation between the forward limit and the $t$ dependence of $H_{v}^{q}(x, t)$ in a fit to the form factors using the sum rule (7). Nevertheless, certain parameters turn out to be relatively stable against variations in the fit, namely

$$
\begin{aligned}
& 1.08 \mathrm{GeV}^{-2}<A_{u}<1.32 \mathrm{GeV}^{-2}, \\
& 1.02 \mathrm{GeV}^{-2}<C_{u}<1.30 \mathrm{GeV}^{-2}, \\
& 0.68 \mathrm{GeV}^{-2}<\alpha_{d}^{\prime}<0.90 \mathrm{GeV}^{-2},
\end{aligned}
$$

with the range of $\alpha_{u}^{\prime}$ being shifted to larger values by $0.1 \mathrm{GeV}^{-2}$. Also, a clear hierarchy between the profile functions for $u$ and $d$ quarks at high $x$ is seen throughout all fits, with $A_{d}>A_{u}$ and $C_{d}>C_{u}$. We also find $D_{d}<0$ and $D_{u}>0$ in all cases. By contrast, the variation in the values of $A_{d}$ is appreciable.

Let us now investigate the variation of the forward limit $e_{v}^{q}(x)$ in our fits. To investigate its behavior at small and large $x$ we have performed the same type of fits as for the PDFs in (45), with a power behavior

$$
\begin{array}{rlrl}
e_{v}^{q}(x) & \sim x^{-\alpha_{q}^{\mathrm{eff}}} \quad & \text { for } 10^{-3}<x<10^{-2}, \\
& \sim(1-x)^{\beta_{q}^{\mathrm{eff}}} \quad \text { for } 0.65<x<0.85 .
\end{array}
$$

For our default fit ABM 1 we find

$$
\begin{array}{ll}
\alpha_{u}^{\mathrm{eff}}=0.526, & \alpha_{d}^{\mathrm{eff}}=0.622, \\
\beta_{u}^{\mathrm{eff}}=4.72, & \beta_{d}^{\mathrm{eff}}=5.44,
\end{array}
$$

where all effective power laws describe $e_{v}^{q}(x)$ within $2 \%$ for the stated $x$ ranges. We did the same exercise for the results of our alternative GPD fits.

In all cases except for fit ABM 2 we find that $\alpha_{d}^{\text {eff }}$ differs from $\alpha$ by at most 0.02 , whereas $\alpha_{u}^{\text {eff }}$ is smaller than $\alpha_{d}^{\text {eff }}$ by about 0.1 . The slight difference between $\alpha_{d}^{\text {eff }}$ and $\alpha$ is due to the factor $(1-x)^{\beta_{d}}$ in the parameterization of $e_{v}^{d}(x)$, 

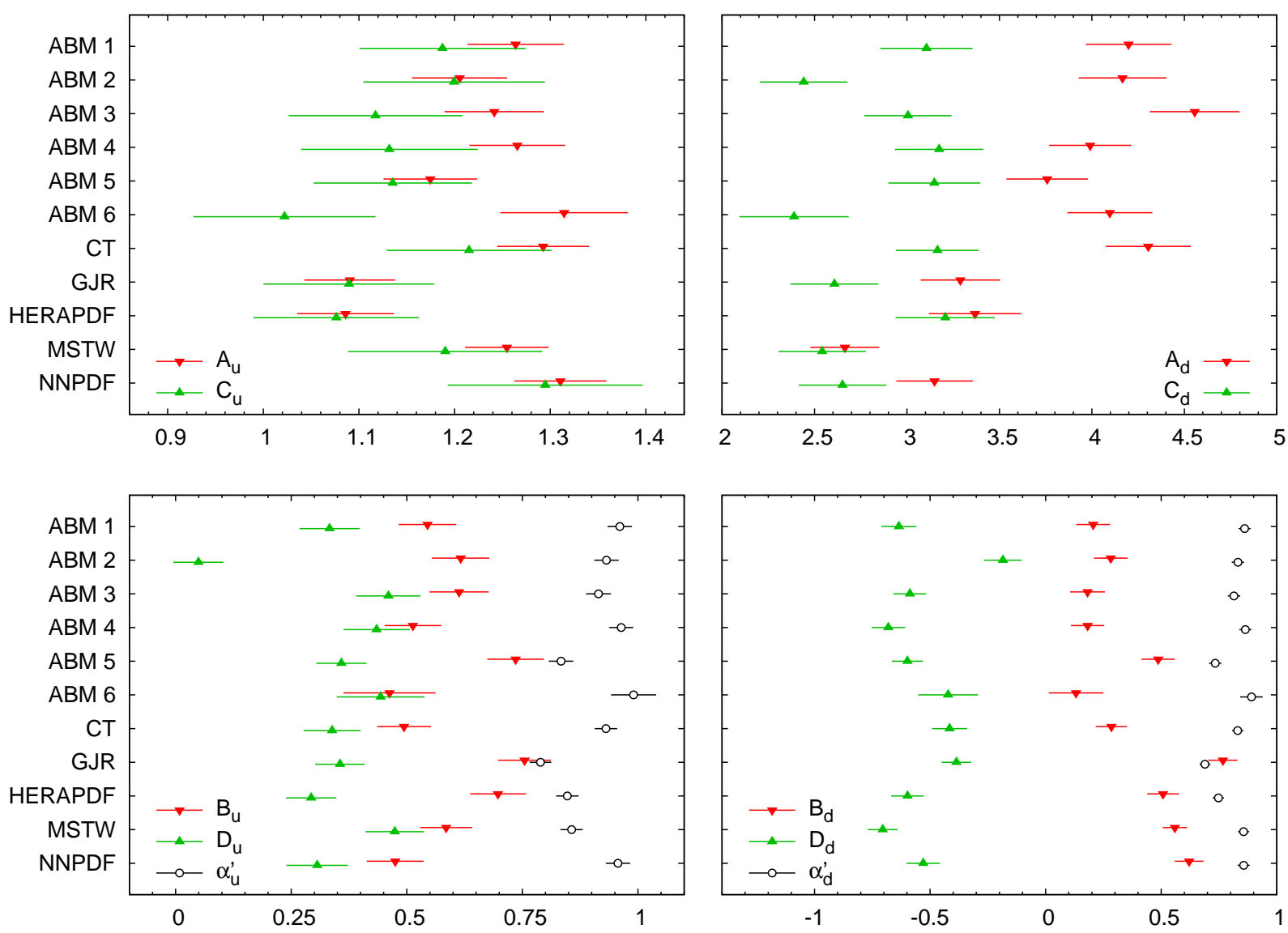

Figure 15: Parameters of the profile functions and their errors obtained in the fits described in table 12. Not shown are the results of fit ABM 0, which refers to GPDs at a different scale $\mu$. Only the error bars for fit ABM 1 include the uncertainty that results from varying $\beta_{u}$ and $\beta_{d}$, which is computed as described in section 6.3.

whereas for $u$ quarks the extra factor $\left(1+\gamma_{u} \sqrt{x}\right)$ with $\gamma_{u}=4$ is responsible for the large difference between $\alpha_{u}^{\text {eff }}$ and $\alpha$. For fit $\mathrm{ABM} 2$, where $\gamma_{u}=\gamma_{d}=4$ we find $\alpha_{u}^{\text {eff }} \approx \alpha_{d}^{\text {eff }} \approx 0.58$, which is smaller than the fitted value of $\alpha$ by about 0.07 . Since fits ABM 1 and ABM 2 describe the data nearly equally well, we cannot conclude whether or not the data favor a slight isospin breaking between the effective small- $x$ powers in $e_{v}^{u}(x)$ and $e_{v}^{d}(x)$.

The range of the effective powers in the different fits is

$$
\begin{aligned}
& 0.52<\alpha_{u}^{\text {eff }}<0.60, \\
& 0.58<\alpha_{d}^{\text {eff }}<0.70 .
\end{aligned}
$$

A comparison with their analogs for the PDFs in table 9 reveals that, for both $u$ and $d$ quarks, $\alpha_{q}^{\text {eff }}$ is clearly larger in $e_{v}^{q}$ than in $q_{v}$ for all our fits (except for the fit with MSTW PDFs, where the effective powers in $e_{v}^{u}$ and $u_{v}$ nearly equal).

Turning to the large- $x$ behavior, we observe that $\beta_{q}^{\text {eff }}$ is slightly smaller than $\beta_{q}$, by at most 0.1 for $u$ quarks and at most 0.2 for $d$ quarks, so that the parameter $\beta_{q}$ gives a good representation of the behavior of $e_{v}^{q}(x)$ at large but not extremely large $x$. The effective power is larger in $e_{v}^{q}$ than in $q_{v}$, with a larger difference for $u$ quarks. In both $q_{v}$ and $e_{v}^{q}$ the effective power of $(1-x)$ is larger for $d$ quarks than for $u$ quarks (with the exception of $e_{v}^{q}$ in the fit using the CT PDFs, whose large- $x$ behavior we find problematic as discussed in section 6.2. 


\begin{tabular}{lcccc}
\hline & $r_{p M}[\mathrm{fm}]$ & $r_{n M}[\mathrm{fm}]$ & $r_{p E}[\mathrm{fm}]$ & $r_{n E}^{2}\left[\mathrm{fm}^{2}\right]$ \\
\hline ABM 1 & $0.832 \pm 0.002$ & $0.854 \pm 0.004$ & $0.838 \pm 0.003$ & $-0.1129 \pm 0.0019$ \\
power law & $0.868 \pm 0.006$ & $0.887 \pm 0.012$ & $0.917 \pm 0.013$ & $-0.1161 \pm 0.0022$ \\
\hline PDG 2012 & $0.777 \pm 0.016$ & $0.862_{-0.008}^{+0.009}$ & $0.8775 \pm 0.0051$ & $-0.1161 \pm 0.0022$ \\
\hline
\end{tabular}

Table 14: Magnetic and charge radii of the proton and neutron obtained in our fits, compared with the values quoted in the Review of Particle Physics (PDG 2012) [36]. We have added in quadrature the statistical and systematic uncertainties given for $r_{p M}$ in PDG 2012.

The effective large- $x$ power for $e_{v}^{q}$ is however not always larger then the one for $q_{v}$ by 1 unit or more, as is suggested by the positivity bound (41), where the factor depending on the profile functions $f_{q}$ and $g_{q}$ behaves like $(1-x)^{2}$ at large $x$. This illustrates that considerations based on the mathematical limit $x \rightarrow 1$ cannot always be taken literally in the region $0.65<x<0.85$, which is of relevance in the integrals that yield the electromagnetic form factors.

\subsection{A note on nucleon radii}

From our fits to the nucleon form factors we can evaluate the associated radii. The values we obtain are given in table 14 . The magnetic radii of proton and neutron are defined as

$$
r_{i M}^{2}=\left.\frac{6}{G_{M}^{i}(0)} \frac{\mathrm{d} G_{M}^{i}(t)}{\mathrm{d} t}\right|_{t=0}
$$

with $i=p, n$. They are compared with their values given in the Review of Particle Physics 2012 (PDG 2012) [36, which are based on the same method as we use here, i.e. on fits of the magnetic form factors measured in elastic electron-nucleon scattering. We observe reasonable agreement for $r_{n M}$ but a clear discrepancy for $r_{p M}$. We do not consider this discrepancy to be a serious shortcoming of our fits. A precise determination of the radii requires form factor measurements at $t$ as small as possible in order to determine the local derivative at $t=0$, together with excellent control over theoretical uncertainties in the form factor extraction and in the fit from which the derivative is extracted. These requirements are clearly not satisfied in our fits, which aim at a global description of all electromagnetic form factors in the full $t$ region where data is available, rather than at a precise description in the vicinity of $t=0$. We do, however, note that the value for $r_{p M}$ quoted by PDG 2012 and reproduced in our table is based on a single experiment [88, which uses a strongly simplified treatment of two-photon exchange in the extraction, and which obtains a significantly smaller result than previous determinations (see the full listing in [36]).

The proton charge radius is defined as

$$
r_{p E}^{2}=\left.6 \frac{\mathrm{d} G_{E}^{p}(t)}{\mathrm{d} t}\right|_{t=0}
$$

We observe a significant discrepancy for this radius between our two fits in table 14 and between our fits and the value given by PDG 2012. We note that the PDG value is a combination of determinations from the Lamb shift in electronic hydrogen and from measurements of $G_{E}^{p}$ in $e p$ scattering. It is fully consistent with the result

$$
r_{p E}=(0.8779 \pm 0.0094) \mathrm{fm}
$$

obtained from the Lamb shift in electronic hydrogen alone (see figure 2 in 89 ), but in strong disagreement with the very precise value

$$
r_{p E}=(0.84184 \pm 0.00067) \mathrm{fm}
$$

that has been extracted from the Lamb shift in muonic hydrogen [90. The origin of this discrepancy is currently unclear, see the discussion and references in [89]. Given this unresolved problem, we have refrained from using a value of $r_{p E}$ as independent input to our form factor fits.

Our results for the squared neutron charge radius, defined in (12), are also given in table 14 


\begin{tabular}{lcccc}
\hline fit & $b_{u 1}[\mathrm{fm}]$ & $b_{d 1}[\mathrm{fm}]$ & $b_{u 2}[\mathrm{fm}]$ & $b_{d 2}[\mathrm{fm}]$ \\
\hline ABM 1 & $0.627 \pm 0.003$ & $0.638 \pm 0.003$ & $0.717 \pm 0.004$ & $0.693 \pm 0.004$ \\
power law & $0.697 \pm 0.011$ & $0.704 \pm 0.011$ & $0.705 \pm 0.007$ & $0.736 \pm 0.016$ \\
\hline
\end{tabular}

Table 15: Average impact parameters 59 for the flavor form factors $F_{i}^{q}$ computed from our fits.

Here the PDG 2012 value, which is obtained from neutron scattering off the shell electrons in nuclear targets, is used as a data point in our fits. As it happens, the power law fit reproduces this value perfectly, whereas the default GPD fit does not. As mentioned above, we do not consider this to be a serious problem, given the global nature of our fits.

For the flavor form factors we can define average impact parameters by

$$
b_{q i}^{2}=\left.\frac{4}{F_{i}^{q}(0)} \frac{\mathrm{d} F_{i}^{q}(t)}{\mathrm{d} t}\right|_{t=0}
$$

with $i=1,2$ and $q=u, d$. Notice the difference between the factor 4 in these twodimensional quantities and the factor 6 in the three-dimensional radii discussed above. The definition (59) is analogous to the one of the impact parameter $\left\langle\boldsymbol{b}^{2}\right\rangle_{x}^{q}$ in (36) but includes an average over the momentum fraction $x$. The results of our fits are given in table 15 for reference. As discussed above, they should not be taken as precision determinations of these quantities, and the discrepancies between the two fits within their respective parametric errors should not be regarded as a problem.

\section{Properties of the fit}

In this section we take a closer look at the GPDs we have extracted from the form factor data. Unless stated otherwise, the results shown are obtained with the default fit ABM 1.

\subsection{Sensitive $x$ range}

To begin with, we investigate which region of $x$ is most important in the form factor integrals (7). We follow our previous work [5] and introduce a

\begin{tabular}{cc}
\hline form factor & $x_{\min }(0)$ \\
\hline$F_{1}^{u}$ & $1.5 \times 10^{-3}$ \\
$F_{1}^{d}$ & $9.2 \times 10^{-4}$ \\
$F_{2}^{u}$ & $2.5 \times 10^{-4}$ \\
$F_{2}^{d}$ & $3.6 \times 10^{-5}$ \\
\hline
\end{tabular}

Table 16: $x_{\min }$ for the flavor form factors at $t=0$, evaluated from the default fit ABM 1.

minimal and a maximal value of $x$ by

$$
\begin{aligned}
\int_{0}^{x_{\min }(t)} \mathrm{d} x K_{v}^{q}(x, t) & =\int_{x_{\max }(t)}^{1} \mathrm{~d} x K_{v}^{q}(x, t) \\
& =0.05 F_{i}^{q}(t)
\end{aligned}
$$

for each of the GPDs $K_{v}^{q}=H_{v}^{q}, E_{v}^{q}$. The $x$ range from $x_{\min }$ to $x_{\max }$ accounts for $90 \%$ of the flavor form factor $F_{i}^{q}$ in the sum rule. For $x<x_{\text {min }}$ and $x>x_{\max }$ the GPDs are obviously not well determined in our analysis. A typical value of $x$ in the integrals is given by the median

$$
\int_{0}^{x_{\mathrm{med}}(t)} \mathrm{d} x K_{v}^{q}(x, t)=0.5 F_{i}^{q}(t) .
$$

In figure 16 we show these quantities for the four flavor form factors. We observe strong correlations between $x$ and $t$. The form factor data at small (large) $t$ determine the GPDs at small (large) $x$. One also sees in figure 16 that the $x$ regions where the GPDs are best determined are rather narrow and even shrink with increasing $-t$. For $\sqrt{-t}=6 \mathrm{GeV}$ the sensitive $x$ values are between 0.6 to 0.9 , while $x_{\max }$ at $t=0$ is between 0.25 and 0.45 depending on the form factor. The values of $x_{\min }$ at $t=0$ are very small and given in table 16 .

The strong $x-t$ correlation is a consequence of our parameterization of the GPDs. Their forward 

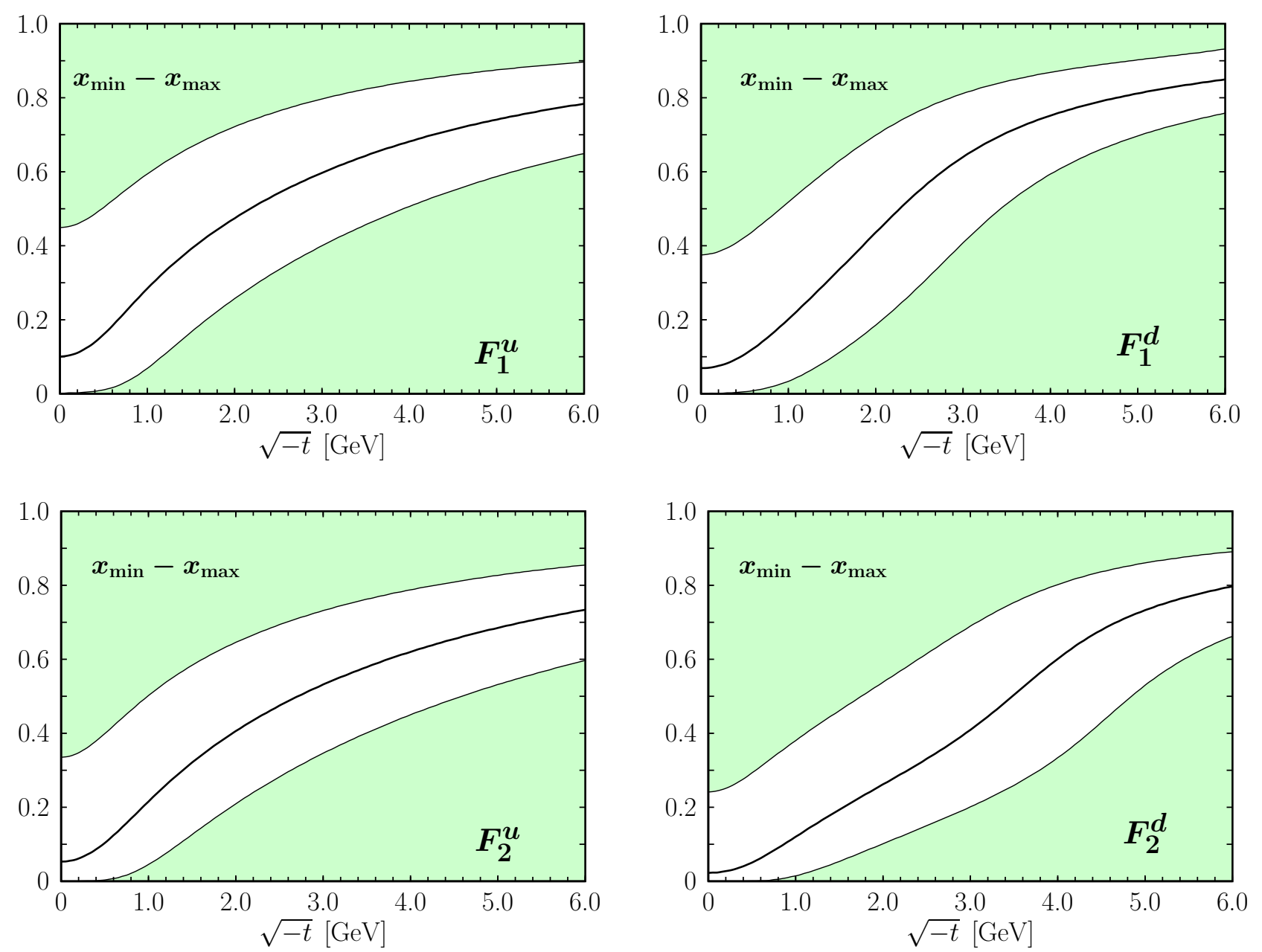

Figure 16: Sensitive (white) and insensitive (shadowed) regions of $x$ for the flavor from factors in the default fit. The upper and lower shaded $x$-regions each account for $5 \%$, the white regions for $90 \%$ of the form factor integrals in (7). The thick solid lines represent the median values of $x$.

limits are singular as $x^{-\alpha}$ which is explicit for $E_{v}^{q}$ and hidden in the PDFs for $H_{v}^{q}$. The values of the Regge intercept $\alpha$ range from 0.33 to 0.62 for our default fit. The profile functions, on the other hand, provide factors $x^{-t \alpha^{\prime}}$. Combining both $x$ factors one has a Regge behavior with a linear trajectory in the exponent of $x$ which crosses zero at $t_{0}=-\alpha / \alpha^{\prime}$. This happens near $-t=0.5 \mathrm{GeV}^{2}$ and turns the singular behavior at $x=0$ for small $t$ into a zero of the GPD at $x=0$ for larger $t$. For yet larger $t$, each of the GPDs develops a pronounced maximum at an $x$ value that increases with $-t$. This behavior is responsible the $x-t$ correlation we observe. In figure 17 we show as an illustration the GPDs at $-t=0.3$ and $6.0 \mathrm{GeV}^{2}$.
We are now in a position to investigate the large- $t$ behavior of the flavor form factors. Since it is controlled by the large- $x$ behavior of the GPDs, we can approximate their forward limits by $(1-x)^{\beta}$ and the profile function by the third term $A(1-x)^{2}$. (For simplicity we use here the same notation for $H_{v}^{q}$ and $E_{v}^{q}$ and drop sub- and superscripts on $\beta$ and $A$.) At sufficiently large $t$ the integral $F(t)=\int \mathrm{d} x K(x, t)$ can be evaluated in the saddle-point approximation, obtained by minimizing the exponent in $K \sim \exp \left[\beta \log (1-x)+t A(1-x)^{2}\right]$ with respect to $x$. One finds [5]

$$
F(t) \sim(-t)^{-(1+\beta) / 2}
$$



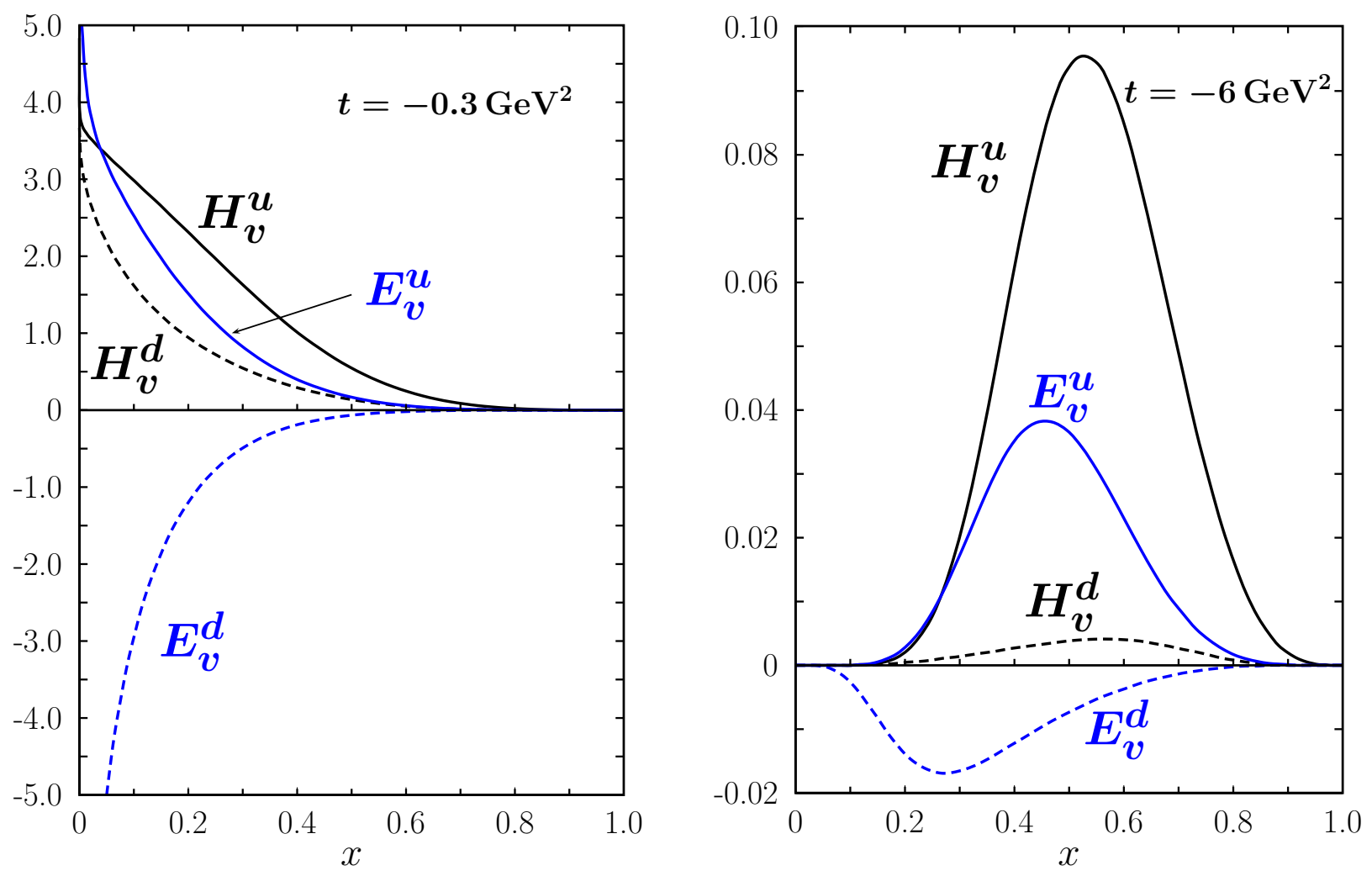

Figure 17: The valence quark GPDs at zero skewness and $-t=0.3$ or $6 \mathrm{GeV}^{2}$. They have been obtained in the default fit and refer to the scale $\mu=2 \mathrm{GeV}$.

with the saddle point being at

$$
x_{s}=1-\sqrt{\beta} / \sqrt{-2 t A} .
$$

We see that the saddle point lies in the so-called soft region

$$
1-x \sim \Lambda / \sqrt{-t}
$$

with $\Lambda$ of hadronic size, where for sufficiently large $t$ the active parton carries most of the proton's momentum while all spectators are soft. The dominance of this soft region has been assumed by Drell and Yan [91] (see also [81]) in order to derive the famous relation between the large- $t$ behavior of the form factors and the large- $x$ behavior of the deep inelastic structure functions.

The derivation of (62) requires the relevant GPD to behave like $(1-x)^{\beta} \exp \left[t A(1-x)^{2}\right]$ in the sensitive $x$ region and to be sufficiently peaked around the saddle point $x_{s}$. Let us see how well (62) works quantitatively, restricting ourselves to the region $\sqrt{-t}<6 \mathrm{GeV}$ where form factor data is available. From tables 9 and 12 we obtain the following powers of $1 /(-t)$ :

$$
\begin{array}{ll}
2.25 \text { for } F_{1}^{u}, & 3.00 \text { for } F_{1}^{d}, \\
2.83 \text { for } F_{2}^{u}, & 3.13 \text { for } F_{2}^{d} .
\end{array}
$$

Both $F_{1}^{u}$ and $F_{2}^{u}$ are well described with these powers when $\sqrt{-t}>4 \mathrm{GeV}$, whereas for $F_{1}^{d}$ and $F_{2}^{d}$ the prediction $(62)$ works only qualitatively. In our fit result for $F_{1}^{d}$ we find a power of 3.35 when $\sqrt{-t}>4 \mathrm{GeV}$, whereas for $F_{2}^{d}$ we see a clear power-law behavior only above $\sqrt{-t}=4.5 \mathrm{GeV}$, with the power being 4.2. Regarding their absolute size, we see in figure 18 that the $d$ quark form factors are suppressed compared to their $u$ quark counterparts for $\sqrt{-t}>2.5 \mathrm{GeV}$ or so. A consequence of this suppression is that

$$
F_{i}^{n} \approx \frac{e_{d}}{e_{u}} F_{i}^{p}
$$

at large $t$. Our results approach this behavior slowly; for $\sqrt{-t}>4 \mathrm{GeV}$ is holds within $10 \%$. 

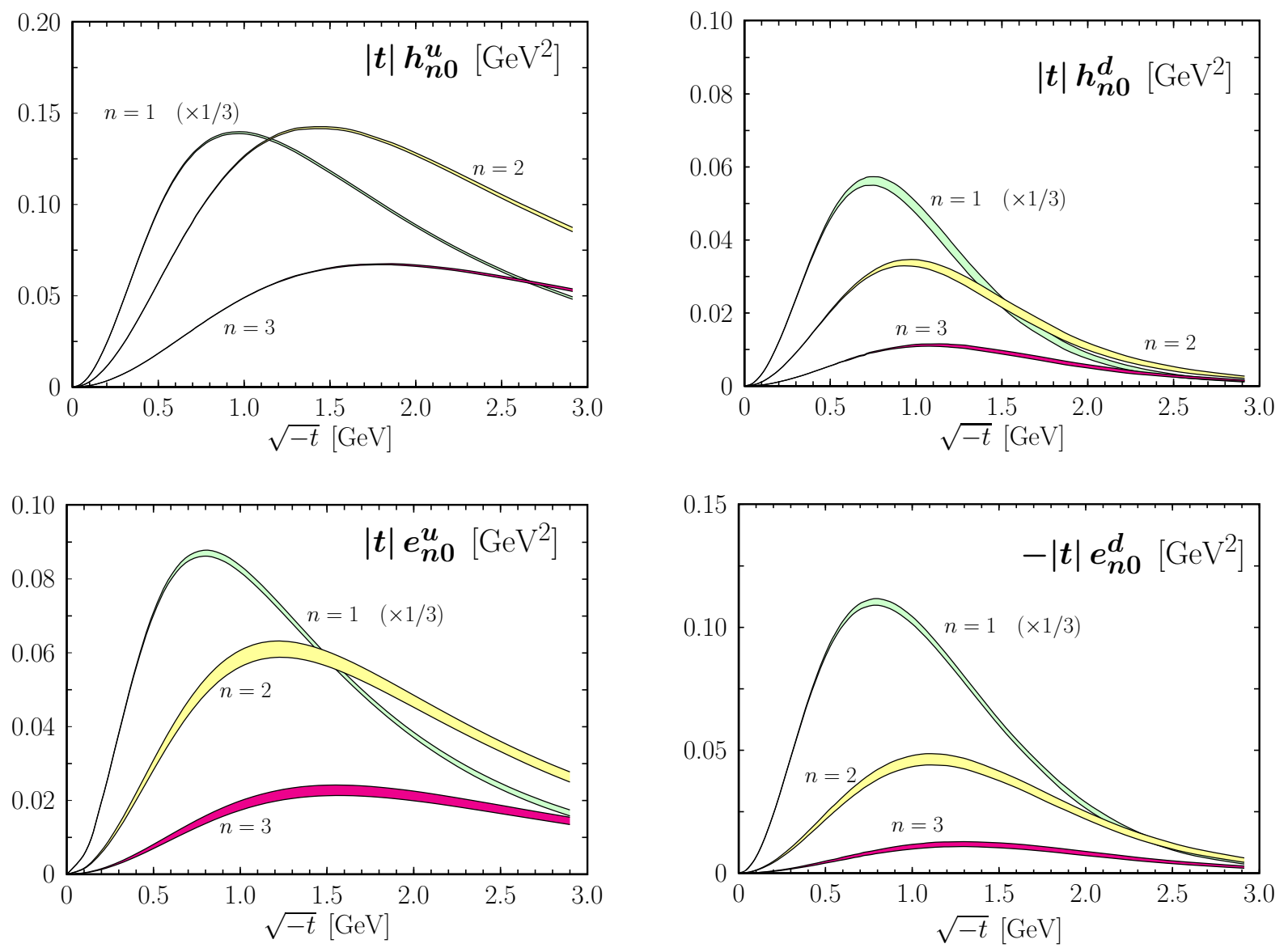

Figure 19: The first three moments of the GPDs obtained in our default fit ABM 1. For better visibility of the higher moments, $h_{10}^{q}$ and $e_{10}^{q}$ have been scaled with $1 / 3$.

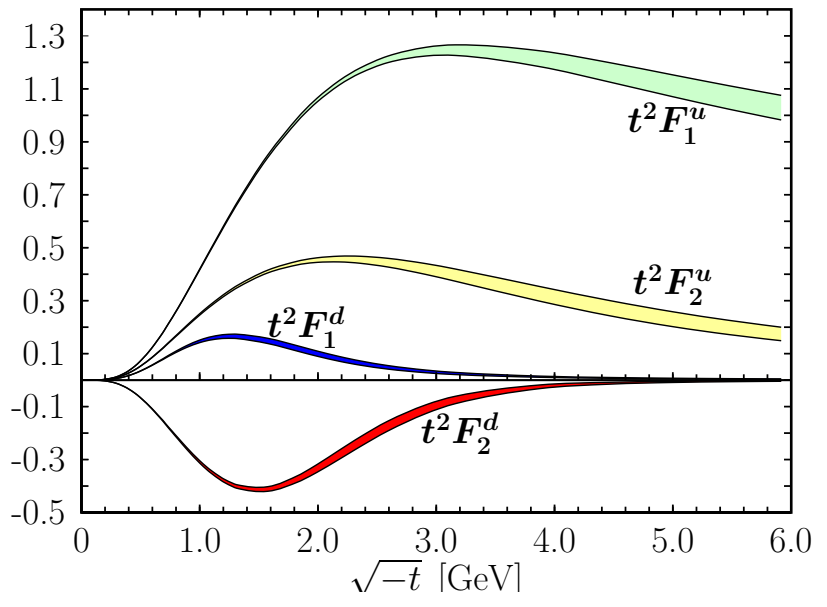

Figure 18: The flavor form factors scaled by $t^{2}$ and shown in units of $\mathrm{GeV}^{4}$, calculated from our default fit ABM 1.

\subsection{Mellin moments and Ji's sum rule}

We have determined the GPDs $H_{v}^{q}$ and $E_{v}^{q}$ by fitting their integrals over $x$ to the form factors $F_{1}^{q}$ and $F_{2}^{q}$ of the electromagnetic current. We now use these GPDs to compute higher moments in $x$. In figure 19 we show the moments

$$
\begin{aligned}
& h_{n 0}^{q}(t)=\int_{0}^{1} \mathrm{~d} x x^{n-1} H_{v}^{q}(x, t), \\
& e_{n 0}^{q}(t)=\int_{0}^{1} \mathrm{~d} x x^{n-1} E_{v}^{q}(x, t),
\end{aligned}
$$

for $n=1,2,3$. In keeping with a standard notation [2, 3], the second subscript 0 indicates that the GPDs are evaluated at zero skewness.

The third moments $h_{30}^{q}$ and $e_{30}^{q}$ are form factors of a twist-two operator containing two covariant derivatives [2, 3]. The second moments $h_{20}^{q}$ and 
$e_{20}^{q}$ are not directly connected to local operators, since the form factors of the relevant twist-two operator with one covariant derivative correspond to the sum and not to the difference of quark and antiquark contributions. In other words, the moments $h_{20}^{q}$ and $e_{20}^{q}$ give the valence contributions (i.e. the difference of quark and antiquark contributions) to the form factors of a twist-two operator, which happens to be the quark part of the energy-momentum tensor.

In figure 19 we observe a strong decrease of the moments with the index $n$, which can be understood from the rather strong decrease of the GPDs with $x$. Moreover, we find that decrease of the moments with $-t$ becomes slower as $n$ increases. Within our ansatz, this is naturally explained by the decrease of the profile functions with $x$, which results in a weaker $t$ slope for the moments that are dominated by higher values of $x$. The same trend has been observed in lattice calculations of GPD moments, see e.g. [92] and the review [93. We refrain, however, from a quantitative comparison with lattice results, since recent studies [94, 95] suggest that in lattice computations of GPD moments it is more involved than previously assumed to achieve full control over the extrapolation to the physical pion mass and over the removal of contributions from excited nucleon states.

We now take a closer look at the second moments at $t=0$. For $H_{v}^{q}$ they just give the momentum fraction carried by the parton species in question. The corresponding numbers for the PDF sets used in our analysis are shown in table 17. We see that these momentum fractions are known with reasonable accuracy, although there is a notable spread between the different PDF sets. We can also assess the importance of sea quark contributions in the momentum sum rule. The numbers in table 17 show that the second moment of the distribution $2(\bar{u}+\bar{d})$, i.e. the momentum fraction carried by the non-strange sea is comparable to the momentum fraction due to the valence $d$ quark distribution. The contribution from strange quarks and antiquarks appears to be relatively unimportant.

The second moments of $E_{v}^{q}$ are given in table 18 . In the first row we give the results of fit ABM 1 with errors computed as described in section 6.3 (i.e. including the variation of $\beta_{u}$ and $\beta_{d}$ ). A more conservative uncertainty estimate is given in the second row, where we take the central values of fit ABM 1 and determine the error from the spread of values from fits ABM 2 to ABM 6 and from the fits with the alternative PDF sets in table 12, The only notable source of uncertainty not included in this estimate is the bias from our functional ansatz for the GPDs themselves, which we cannot assess within our present study. A graphical display of the second moments obtained in the different fits is shown in figure 20. We find that $e_{20}^{u}(0)$ and $e_{20}^{d}(0)$ are determined rather well. Their sum is found to be very small; within its error it may be positive or negative.

In the third row of table 18 we give the second moments from fit $\mathrm{ABM} 0$, which refers to the lower scale $\mu=1 \mathrm{GeV}$. The uncertainties are computed as for the first row, including again the variation of $\beta_{u}$ and $\beta_{d}$. The moments $e_{20}^{u}(0)$ and $e_{20}^{d}(0)$ are larger in absolute magnitude than at $\mu=2 \mathrm{GeV}$, which is in line with what is expected from scale evolution. The isosinglet combination $e_{20}^{u+d}(0)$ remains very small.

According to Ji [96, the sum

$$
2 J_{v}^{q}=h_{20}^{q}(0)+e_{20}^{q}(0)
$$

of second moments gives two times the angular momentum carried by quarks of flavor $q$, minus the corresponding antiquark contribution. Its values for our different fits are shown in figure 20 and listed in table 19 with error estimates analogous to those in table 18 . We find that at $\mu=2 \mathrm{GeV}$ valence $u$ quarks carry about half of

\begin{tabular}{lcc}
\hline fits & $2 J_{v}^{u}$ & $2 J_{v}^{d}$ \\
\hline ABM 1 & $0.460_{-0.010}^{+0.006}$ & $-0.007_{-0.006}^{+0.008}$ \\
all fits & $0.460_{-0.048}^{+0.018}$ & $-0.007_{-0.033}^{+0.021}$ \\
\hline ABM 0 & $0.560_{-0.010}^{+0.009}$ & $-0.019_{-0.009}^{+0.009}$ \\
\hline
\end{tabular}

Table 19: Results for the total angular momentum of quarks minus the contribution from antiquarks according to Ji's sum rule. For further explanations see table 18 . 


\begin{tabular}{lccccccc}
\hline PDF & $u-\bar{u}$ & $d-\bar{d}$ & $2 \bar{u}$ & $2 \bar{d}$ & $2(\bar{u}+\bar{d})$ & $s+\bar{s}$ & $s-\bar{s}$ \\
\hline ABM & 0.297 & 0.115 & 0.062 & 0.077 & 0.139 & 0.035 & 0 \\
CT & 0.287 & 0.118 & 0.058 & 0.072 & 0.130 & 0.040 & 0 \\
GJR & 0.280 & 0.116 & 0.064 & 0.080 & 0.144 & 0.021 & 0 \\
HERAPDF & 0.284 & 0.105 & 0.074 & 0.091 & 0.165 & 0.044 & 0 \\
MSTW & 0.282 & 0.115 & 0.064 & 0.076 & 0.140 & 0.033 & 0.0019 \\
NNPDF & 0.290 & 0.124 & 0.059 & 0.074 & 0.133 & 0.020 & 0.0029 \\
\hline
\end{tabular}

Table 17: Second moments of the parton densities specified in table 9, given at scale $\mu=2 \mathrm{GeV}$.

\begin{tabular}{lcccc}
\hline fits & $e_{20}^{u}(0)$ & $e_{20}^{d}(0)$ & $e_{20}^{u+d}(0)$ & $e_{20}^{u-d}(0)$ \\
\hline ABM 1 & $0.163_{-0.006}^{+0.010}$ & $-0.122_{-0.006}^{+0.008}$ & $0.041_{-0.010}^{+0.008}$ & $0.284_{-0.012}^{+0.012}$ \\
all fits & $0.163_{-0.032}^{+0.018}$ & $-0.122_{-0.033}^{+0.028}$ & $0.041_{-0.053}^{+0.011}$ & $0.284_{-0.060}^{+0.040}$ \\
\hline ABM 0 & $0.204_{-0.010}^{+0.009}$ & $-0.156_{-0.009}^{+0.009}$ & $0.048_{-0.010}^{+0.006}$ & $0.360_{-0.017}^{+0.017}$ \\
\hline
\end{tabular}

Table 18: Results for second moments of $E_{v}^{q}$ at $t=0$. The central values in the rows labeled ABM 1 and "all fits" correspond to the default fit and refer to $\mu=2 \mathrm{GeV}$, with two different error estimates as described in the text. The last row gives the moments obtained from the fit ABM 0 and refers to $\mu=1 \mathrm{GeV}$.
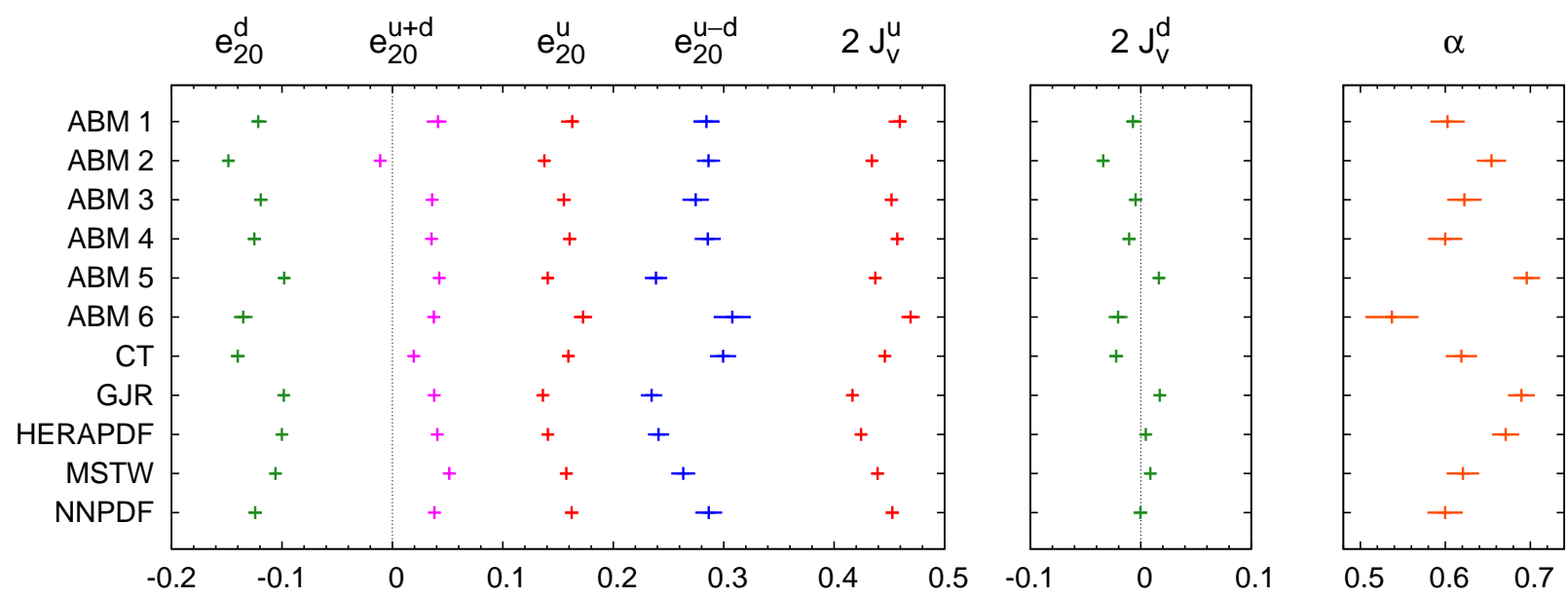

Figure 20: Results for the second moments of GPDs at $t=0$ and for the parameter $\alpha$ from the GPD fits specified in table 12 . Only the error bars for fit ABM 1 include the uncertainty that results from varying $\beta_{u}$ and $\beta_{d}$, which is computed as described in section 6.3 . 
the nucleon spin. By contrast, the contribution of valence $d$ quarks is very small, even consistent with zero, as a result of the cancellation between $h_{20}^{u}(0)$ and $e_{20}^{u}(0)$. Let us emphasize that we cannot separately determine the contributions from antiquarks to the nucleon spin in our analysis. If the second moments of the PDFs are any guidance, then this contribution may not be negligible.

Within errors, our numbers for $J_{v}^{u}=J^{u-\bar{u}}$ and $J_{v}^{d}=J^{d-\bar{d}}$ are consistent with the results in [97, where these quantities are estimated using a model dependent connection between GPDs and Sivers distributions (see section 8.3). Our numbers are also close to the corresponding values of $J^{u+\bar{u}}$ and $J^{d+\bar{d}}$ obtained in lattice calculations [93], although such a comparison should be taken with care given the comments we made earlier in this section. An overview of other determinations of $J^{u+\bar{u}}$ and $J^{d+\bar{d}}$ can be found in $[98$.

\subsection{Impact parameter distributions}

In section 6.1 we explained how the GPDs $H_{v}^{q}(x, t)$ and $E_{v}^{q}(x, t)$, after Fourier transformation to impact parameter space, give information about the spatial distribution of partons with momentum fraction $x$ in the impact parameter plane. Let us now see how this plays out quantitatively for our default fit ABM 1.

The impact parameter $\boldsymbol{b}$ introduced in section 6.1 gives the distance of a parton from the center of the proton, where the center is determined by the transverse positions of all proton constituents, weighted with their momentum fraction [79]. The impact parameter of a parton with $x$ close to 1 thus tends to coincide with the center of the proton, and a better quantity to assess the overall proton size in that case is the distance $\boldsymbol{b} /(1-x)$ between the struck parton and the center of all spectator partons in the transverse plane. Following [5], we introduce the average

$$
d_{q}(x)=\frac{\sqrt{\left\langle\boldsymbol{b}^{2}\right\rangle_{x}^{q}}}{1-x}=\frac{2 \sqrt{f_{q}(x)}}{1-x} .
$$

of this distance. It is plausible to require that the proton remains of finite transverse size for configurations where one parton has momentum fraction
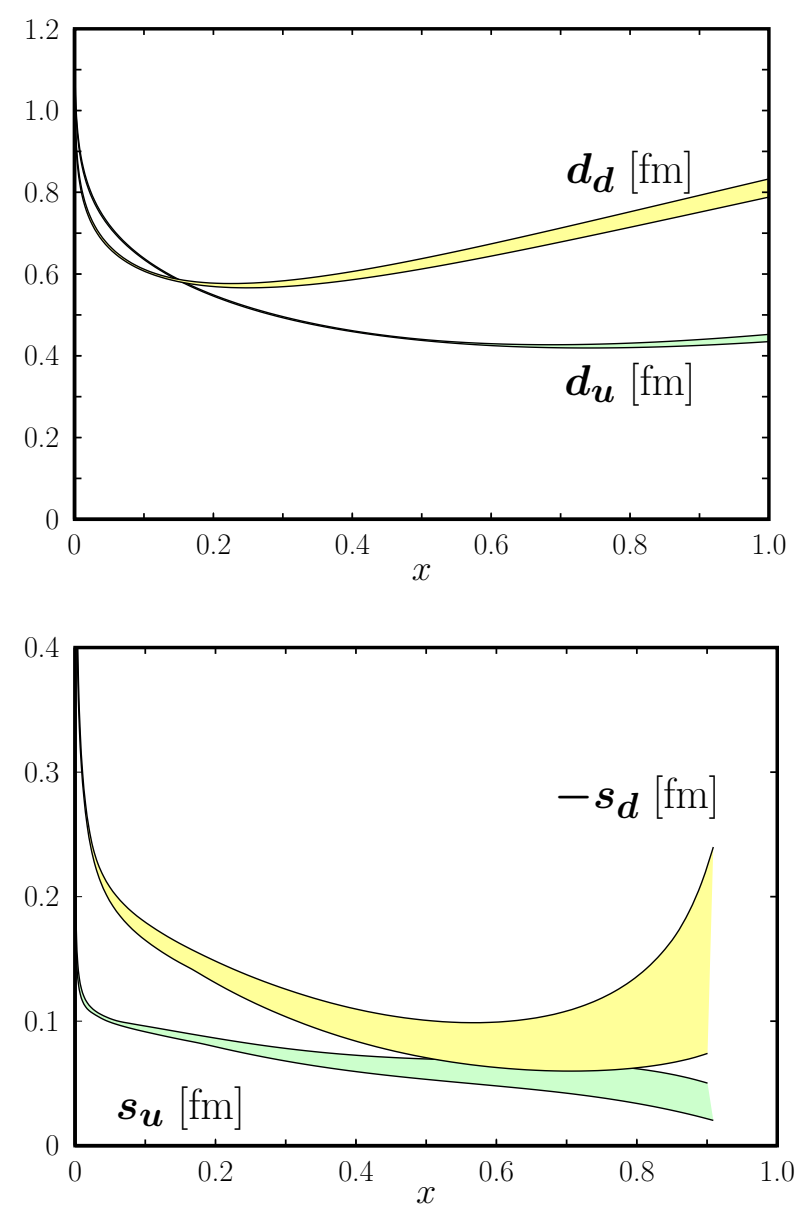

Figure 21: The average distance $d_{q}$ between the active quark and the cluster of spectators for $u$ and $d$ quarks, and the average shift $s_{q}$ of this distance along $y$ in a proton polarized along the $x$ direction.

$x \rightarrow 1$, which implies that $\left\langle\boldsymbol{b}^{2}\right\rangle_{x}^{q} \sim(1-x)^{2}$ in that limit as we anticipated in section 6.1

In figure 21 this distance is shown for $u$ and $d$ quarks. The values of $d_{q}$ appear reasonable for an estimate of the transverse size of the proton. In consistency with our earlier analysis [5], we find that $d_{d}$ is clearly larger than $d_{u}$ for medium to large $x$. The comparison of the relevant parameters $A_{u}$ and $A_{d}$ in the profile function (see section 6.4 shows that this trend is seen in all our GPD fits. Due to the term with $\alpha_{q}^{\prime} \log (1 / x)$ in the profile function, the distance between the struck quark and the cluster of spectators growths logarithmically when $x$ goes to zero. 
In analogy to 69 we introduce the average size

$$
s_{q}(x)=\frac{\left\langle b^{y}\right\rangle_{x}^{q}}{1-x}=\frac{1}{2 m} \frac{e_{v}^{q}(x)}{q_{v}(x)} \frac{1}{1-x}
$$

of the shift in the distance between the struck quark and the spectator system that is induced by transverse proton polarization. As is seen in figure 21, this shift is significantly nonzero and larger in size for $d$ quarks than for $u$ quarks. For $x \rightarrow 1$ the numerical values we find for $s_{q}$ are very uncertain, which is due to the corresponding uncertainties in the parton densities we discussed in section 6.2. We therefore limit the plot of $s_{q}$ to the region $x<0.9$.

Observing that the r.h.s. of the positivity bound (41) assumes its maximum for $g_{q}(x) / f_{q}(x)=3 / 4$ and omitting $\Delta q_{v}(x)$, we obtain the bound

$$
\left|s_{q}(x)\right|<0.38 d_{q}(x) .
$$

We note that the weaker bound $\left|s_{q}(x)\right| \leq 0.5 d_{q}(x)$ can be derived directly from the general form (40, independently of our exponential ansatz (33) for the $t$ dependence of the GPDs, see [81]. For our default fit we find that in the interval $0.15<$ $x<0.9$ the ratio $\left|s_{q}(x)\right| / d_{q}(x)$ is below 0.16 for $u$ quarks and below 0.27 for $d$ quarks, so that the bound (71) on the displacement of quarks due to transverse proton polarization is far from saturated in the $x$ region where it should be applicable.

In the phenomenology of deeply virtual Compton scattering (DVCS) and of hard exclusive meson production, a Regge-type approximation of the profile function $f_{q}$, namely

$$
f_{R}(x)=\alpha_{R}^{\prime} \log (1 / x)+B_{R}
$$

is frequently used. In figure 22 we compare the distance $d_{u}$ obtained with this approximation and its counterpart calculated from the profile function (44) in our default fit. We also show the separate contributions of the Regge terms $\left[\alpha_{u}^{\prime} \log (1 / x)+B_{u}\right](1-x)^{3}$ and of the large- $x$ term $A_{u} x(1-x)^{2}$ in $f_{u}(x)$. The Regge-type approximation $f_{R}$ agrees well with $f_{u}$ at small $x$, which is expected from their definitions (small deviations are due to the slightly different parameters in $f_{R}$

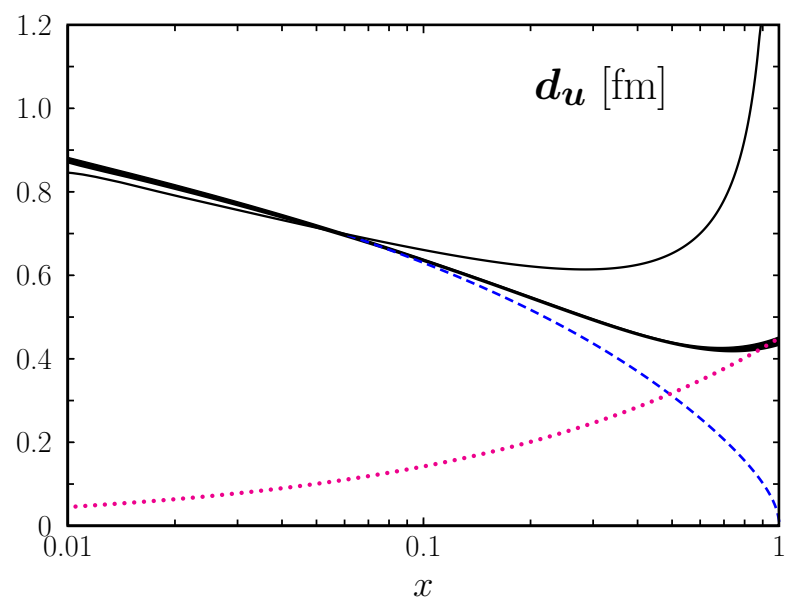

Figure 22: Comparison of the distance $d_{u}$ evaluated with the Regge-type profile function (72) (thin solid line) and with $f_{q}$ in our default fit (thick solid line). We also show the separate contributions from the Regge terms (dashed) and the large- $x$ term (dotted) in $f_{q}$, as specified in the text. The Regge-type profile function is evaluated with the parameters $\alpha_{R}^{\prime}=0.9 \mathrm{GeV}^{-2}$ and $B_{R}=0$ taken from [100.

and our fit). For growing $x$ the Regge-type approximation increasingly deviates from the distance evaluated with our profile function $f_{u}$, and in the limit $x \rightarrow 1$ it increases as $1 /(1-x)$, which is clearly an unphysical behavior. The form (72) is thus suitable at small $x$ but should not be used in the large- $x$ region.

\section{Applications}

\subsection{Axial form factor}

The axial form factor of the nucleon, $F_{A}(t)$, is related by the sum rule (11) to the quark helicity dependent GPDs. Data on the axial form factor are scarce (mainly limited to $-t<1 \mathrm{GeV}^{2}$ ) and show a considerable spread, see for instance the review [101]. The measurement [102], which covers the largest $t$-range, namely $0.1 \mathrm{GeV}^{2}<-t<3 \mathrm{GeV}^{2}$, is presented in form of a dipole parameterization $F_{A}(t)=F_{A}(0) /\left(1-t / M_{A}^{2}\right)^{2}$ with parameters $F_{A}(0)=1.23 \pm 0.01$ and $M_{A}=\left(1.05_{-0.16}^{+0.12}\right) \mathrm{GeV}$. 


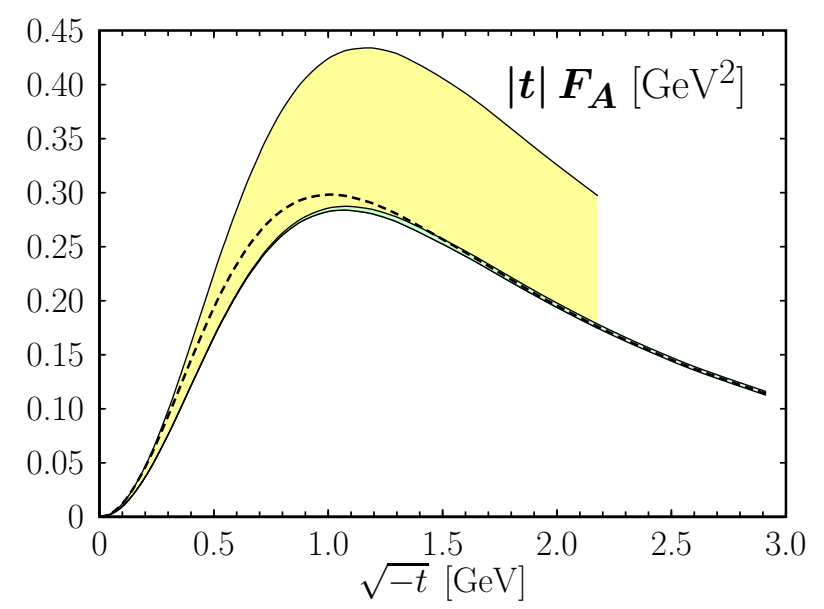

Figure 23: The axial form factor $F_{A}$ of the nucleon. The data [102] are represented by their dipole parameterization as a yellow band. The GPD result is evaluated with the default fit for $f_{u}(x)$ and $f_{d}(x)$ and the polarized PDFs from [80]. The dashed line represents our result for $F_{A}$ including an estimate of the sea quarks, as explained in the text.

In view of this situation we do not attempt to fit the GPDs for polarized quarks to the data on the axial form factor. Instead, we use the ansatz (34) for $\widetilde{H}_{v}^{q}(x, t)$ with the profile functions $f_{u}(x)$ and $f_{d}(x)$ fixed by our fit of the unpolarized GPDs. The polarized parton distributions $\Delta q_{v}$ for valence quarks are taken from the analysis of DSSV [80], as we already did when evaluating the positivity constraint (41).

With this ansatz for $\widetilde{H}_{v}^{q}$ the axial form factor is evaluated from the sum rule (11), neglecting the antiquark contribution for the time being. The results are compared to the dipole fit of [102] in figure 23. One sees that our simple model of $\widetilde{H}_{v}^{q}$ is compatible with experiment, although it is at the lower edge of the large uncertainty band of the dipole fit.

In order to get a feeling for the possible size of the antiquark contribution in the sum rule for $F_{A}$, we use the parameterization (34) also for the polarized sea quark distribution $H^{\bar{q}}$. Admittedly, this is an ad hoc ansatz and should not be over-interpreted. Since the isotriplet combination $\Delta \bar{u}(x)-\Delta \bar{d}(x)$ of forward densities is not very small in the DSSV analysis, the effect of the sea quarks on the axial form factor is noticeable for $-t$ below $1 \mathrm{GeV}^{2}$. The agreement with the dipole fit improves within this estimate, as is seen in figure 23.

A more general ansatz than (34) is the exponential form $\widetilde{H}_{v}^{q}(x, t)=\Delta q_{v}(x) \exp \left[t \tilde{f}_{q}(x)\right]$ with a profile function of its own. The density interpretation of the impact parameter distributions $q_{v}\left(x, \boldsymbol{b}^{2}\right) \pm \Delta q_{v}\left(x, \boldsymbol{b}^{2}\right)$ for valence quarks with definite helicity implies the bound $\tilde{f}_{q}(x) \leq f_{q}(x)$ in the region where antiquarks can be neglected. Taking $\tilde{f}_{q}(x)<f_{q}(x)$ instead of $\tilde{f}_{q}(x)=f_{q}(x)$ would increase the integral giving the axial form factor and could thus improve the agreement with the dipole fit, especially at higher $t$. We will, however, not pursue this possibility in the present work.

\subsection{Compton form factors}

As argued in [103, 104, the amplitude for wideangle Compton scattering (i.e. Compton scattering at large values of the Mandelstam variables $s, t$ and $u$ ) factorizes into a hard subprocesses $\gamma q \rightarrow \gamma q$ and form factors given by the $1 / x$ moments of GPDs:

$$
\begin{aligned}
& R_{V}=\sum_{q} e_{q}^{2} \int_{0}^{1} \frac{\mathrm{d} x}{x}\left[H_{v}^{q}(x, t)+2 H^{\bar{q}}(x, t)\right], \\
& R_{T}=\sum_{q} e_{q}^{2} \int_{0}^{1} \frac{\mathrm{d} x}{x}\left[E_{v}^{q}(x, t)+2 E^{\bar{q}}(x, t)\right], \\
& R_{A}=\sum_{q} e_{q}^{2} \int_{0}^{1} \frac{\mathrm{d} x}{x}\left[\widetilde{H}_{v}^{q}(x, t)+2 \widetilde{H}^{\bar{q}}(x, t)\right] .
\end{aligned}
$$

This factorization, which bears some similarity to the handbag factorization of DVCS, is formulated in a symmetric frame where the skewness $\xi$ is zero. The form factors are mildly scale dependent as discussed in [5]. We evaluate these Compton from factors from our default fit of the valence-quark GPDs at the scale $\mu=2 \mathrm{GeV}$, taking again the ansatz $(34)$ for $\widetilde{H}_{v}^{q}$. The results and their parametric uncertainties are shown in figure 24 for $-t>2 \mathrm{GeV}^{2}$. They are rather similar to those obtained in our previous work [5], except that $R_{T}$ in the present analysis is somewhat larger at small $t$ and falls off slightly faster as $-t$ 


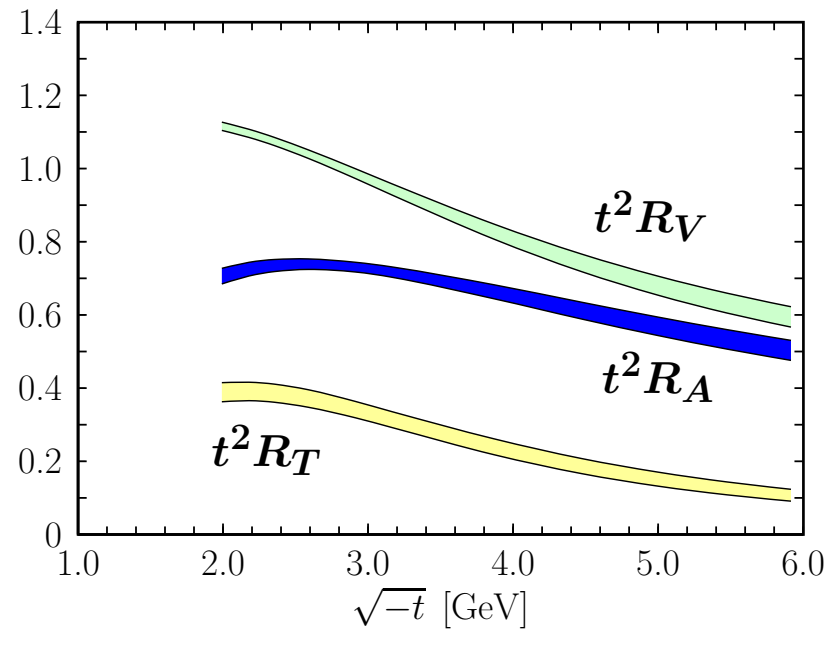

Figure 24: The Compton form factors evaluated from our default fit at the scale $\mu=2 \mathrm{GeV}$. They are scaled by $t^{2}$ and shown in units of $\mathrm{GeV}^{4}$.

increases. The sea quark contribution to these form factors can be safely neglected since we are only interested in the large $t$ region (c.f. our estimate of the sea quark contribution to the axial form factors in section 8.1.

In the handbag approach, the unpolarized cross section for wide-angle Compton scattering reads 104

$$
\begin{aligned}
\frac{\mathrm{d} \sigma}{\mathrm{d} t}=\frac{\pi \alpha_{\mathrm{em}}^{2}}{s^{2}} \frac{(s-u)^{2}}{-u s} & {\left[R_{V}^{2}(t)-\frac{t}{4 m^{2}} R_{T}^{2}(t)\right.} \\
& \left.+\frac{t^{2}}{(s-u)^{2}} R_{A}^{2}(t)\right] .
\end{aligned}
$$

In figure 25 we plot this quantity for $s=10.92$ and $20 \mathrm{GeV}^{2}$ and compare with the Hall A data [105] from JLab. The theoretical results include next-to-leading order QCD corrections [5, 106] and an estimate of the uncertainties due to the finite proton mass as specified in [107]. The latter uncertainties are responsible for the error bands, because the parametric errors on the Compton form factors resulting from our GPD fit are rather small. Despite the fact that the agreement with the Hall A data is not perfect, in particular in the forward hemisphere, we consider our result as a remarkable success: in a parameter-free calculation the deviations between experiment and theory are less than about $30 \%$.

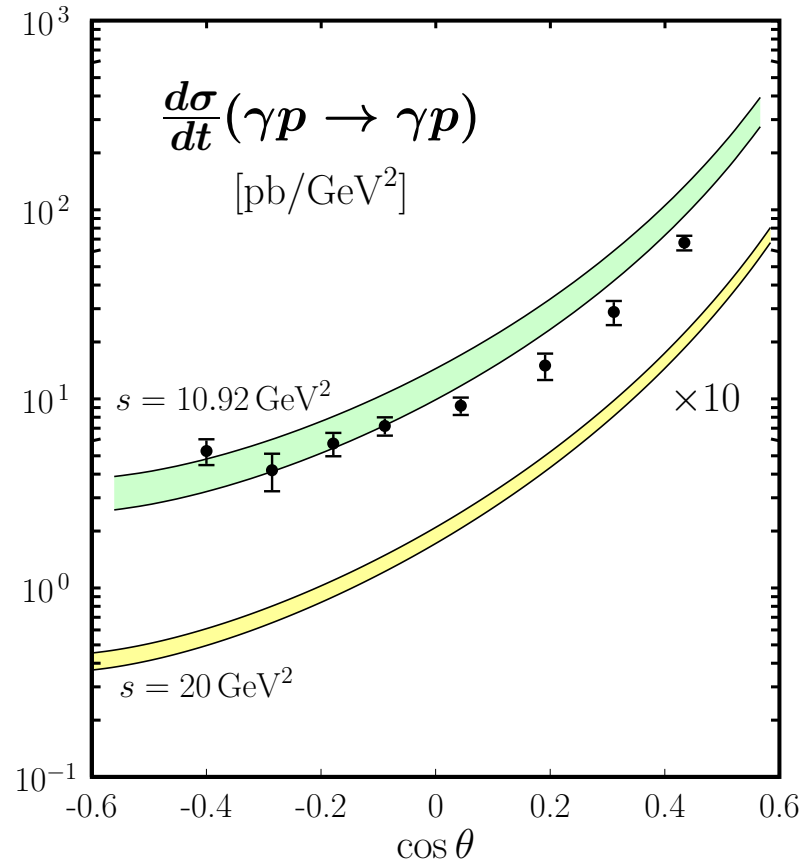

Figure 25: The Compton cross section at $s=$ 10.92 and $20 \mathrm{GeV}^{2}$, evaluated from the Compton form factors shown in figure 24 . The data points for $s=10.92 \mathrm{GeV}^{2}$ are from $[105]$.

Another interesting quantity is the correlation parameter $A_{L L}\left(K_{L L}\right)$ between the helicities of the incoming photon and the incoming (outgoing) proton [106, 108. As a consequence of the neglect of quark masses, one has $A_{L L}=K_{L L}$ in the handbag approach. To a good approximation, the correlation parameters are given by the ratio of $R_{A} / R_{V}$ times a known factor. There is a measurement of $K_{L L}$ for $s=6.9 \mathrm{GeV}^{2}$ and $-t=4 \mathrm{GeV}^{2}$ [109], but we deem the corresponding value $-u=1.1 \mathrm{GeV}^{2}$ to be too low for applying the handbag approach. The situation should improve with future measurements of Compton scattering at higher energies.

\subsection{Chromodynamic lensing and the Sivers distribution}

The Sivers distribution quantifies the anisotropy in the transverse-momentum distribution of unpolarized partons inside a proton that is polarized in the transverse plane. Its very existence 
offers deep insights into the dynamics of partons in QCD: the distribution is naively time-reversal odd and can only be nonzero due to interactions of the spectator partons that single out a direction in time [110]. These interactions play a role in every type of parton distribution; technically they are described by Wilson lines if one works in a covariant gauge [111.

We have already seen that in a transversely polarized proton there is a sideways shift in the spatial distribution of partons, which is described by the term with $e_{v}^{q}\left(x, \boldsymbol{b}^{2}\right)$ in (38). The idea of chromodynamic lensing [112] is that this anisotropy in transverse space induces an anisotropic distribution of the transverse parton momentum via the spectator interactions just mentioned. These interactions are of non-perturbative nature, and to date the lensing effect cannot be computed from first principles in QCD. It can however be explored in simple model calculations, where typically the spectator system is approximated by a diquark and its interactions are treated as gluon exchange in perturbation theory, see e.g. [113, 114.

In the recent study 97 an ansatz for the lensing effect was used to compute GPDs from a phenomenological extraction of the Sivers distributions. In this work we take the opposite approach and combine a simple model for chromodynamic lensing with our fit result for the distribution $e_{v}^{q}(x, \boldsymbol{b})$, thus obtaining an estimate for the Sivers distribution of valence $u$ and $d$ quarks. A useful quantity for this purpose is the first $k_{T}^{2}$ moment of the Sivers function, defined as

$$
f_{1 T}^{\perp(1), q-\bar{q}}(x)=\int \mathrm{d}^{2} \boldsymbol{k} \frac{\boldsymbol{k}^{2}}{2 m^{2}} f_{1 T}^{\perp, q-\bar{q}}\left(x, \boldsymbol{k}^{2}\right),
$$

where we abbreviate

$$
f_{1 T}^{\perp, q-\bar{q}}\left(x, \boldsymbol{k}^{2}\right)=f_{1 T}^{\perp, q}\left(x, \boldsymbol{k}^{2}\right)-f_{1 T}^{\perp, \bar{q}}\left(x, \boldsymbol{k}^{2}\right)
$$

and use the standard definition of the Sivers distribution [115]. We take a model that involves a scalar diquark and perturbative one-gluon exchange [113, which gives

$$
\begin{aligned}
& f_{1 T}^{\perp(1), q-\bar{q}}(x) \\
& \quad=-\pi \alpha_{s} C_{F} \frac{1}{2 m^{2}} \int \frac{\mathrm{d}^{2} \boldsymbol{l}}{(2 \pi)^{2}} \frac{1}{\boldsymbol{l}^{2}+m_{g}^{2}}
\end{aligned}
$$

$$
\begin{aligned}
& \times \frac{\boldsymbol{l}^{2}}{1-x} E_{v}^{q}\left(x,-\frac{\boldsymbol{l}^{2}}{(1-x)^{2}}\right) \\
= & -\int \mathrm{d}^{2} \boldsymbol{b} \frac{b^{j}}{2 m^{2}} I^{j}\left(\frac{\boldsymbol{b}}{1-x}\right) \frac{\partial e_{v}^{q}\left(x, \boldsymbol{b}^{2}\right)}{\partial \boldsymbol{b}^{2}} .
\end{aligned}
$$

The so-called lensing function thus reads

$$
\begin{aligned}
I^{j}(\boldsymbol{b}) & =2 \pi \alpha_{s} C_{F} \frac{\partial}{\partial b^{j}} \int \frac{\mathrm{d}^{2} \boldsymbol{l}}{(2 \pi)^{2}} \frac{e^{-i \boldsymbol{b} \boldsymbol{l}}}{\boldsymbol{l}^{2}+m_{g}^{2}} \\
& =-\alpha_{s} C_{F} \frac{b^{j}}{b^{2}}\left[b m_{g} K_{1}\left(b m_{g}\right)\right],
\end{aligned}
$$

where $b=\sqrt{\boldsymbol{b}^{2}}$. We note that the expression in squared brackets tends to 1 for $m_{g} \rightarrow 0$. These results follow from equations (87), (89) and (90) in [113] if we introduce a mass $m_{g}$ in the gluon propagator and if we change $e_{q} e_{s} \rightarrow-g^{2} C_{F}=$ $-4 \pi \alpha_{s} C_{F}$, as is appropriate when going from an Abelian gluon model to color $\mathrm{SU}(3)$ with a color singlet target. We allow for a gluon mass in order to explore non-perturbative effects at least in a very simple fashion. With our exponential ansatz (33) for the $t$-dependence of $E_{v}^{q}$, we obtain

$$
\begin{aligned}
& f_{1 T}^{\perp(1), q-\bar{q}}(x) \\
& \quad=-\alpha_{s} C_{F} \frac{(1-x) e_{v}^{q}(x)}{8 m^{2} g_{q}(x)} \chi\left[\frac{m_{g}^{2} g_{q}(x)}{(1-x)^{2}}\right],
\end{aligned}
$$

where the auxiliary function

$$
\chi(z)=1-z e^{z} \int_{1}^{\infty} \frac{d u}{u} e^{-u z}
$$

behaves like $1-z \log (1 / z)$ for $z \rightarrow 0$ and like $1 / z$ for $z \rightarrow \infty$. With the definition (69) of the distance function $d_{q}(x)$ this implies

$$
\left|f_{1 T}^{\perp(1), q-\bar{q}}(x)\right| \leq \frac{\alpha_{s} C_{F}}{2 m^{2} d_{q}^{2}(x)} \frac{f_{q}(x)}{g_{q}(x)} \frac{e_{v}^{q}(x)}{1-x} .
$$

In our numerical study we set $\alpha_{s}=1$, bearing in mind that the typical scale of the one-gluon exchange is non-perturbative, as can easily be seen in (77). Even without a small $\alpha_{s}$, the $k_{T}^{2}$ moment of the Sivers function is suppressed by the factor $1 /\left[2 m^{2} d_{q}^{2}(x)\right]$, given that the distance $d_{q}(x)$ is significantly larger than the Compton wavelength $1 / m \approx 0.2 \mathrm{fm}$ of the proton (see figure 21).

The model leading to (79) does not include evolution effects, neither for the Sivers function 
nor for $e_{v}^{q}$ (the two types of function actually evolve quite differently). We evaluate $(79)$ at scale $\mu=1 \mathrm{GeV}$ rather than at $\mu=2 \mathrm{GeV}$, so as to be closer to the non-perturbative region that is natural for the model. We hence use the distribution $e_{v}^{q}$ obtained in the fit $\mathrm{ABM} 0$ (see table 12 rather than the one from our default fit. The result is shown in figure 26 for three representative values of the gluon mass $m_{g}$. It is compared with the recent extraction [116] of the Sivers distribution from semi-inclusive deep inelastic scattering data. We observe overall agreement in sign and order of magnitude between our model results and the phenomenological extraction, but there are clear discrepancies as well, most notably for the relative size of $u$ and $d$ quark distributions and for the detailed shape in $x$. As a word of caution we note that the extraction in [116] (as well as any other current extraction of the Sivers function) is subject to important theoretical uncertainties. Our overall assessment is however that the simple model for chromodynamic lensing we have used cannot be expected to yield precise predictions.

\subsection{Skewness dependence}

The sum rules relating GPDs to electromagnetic form factors do not contain information about the dependence of the GPDs on the skewness parameter $\xi$. We have therefore used these sum rules at $\xi=0$, see (7) and (8), because at this point GPDs admit a density interpretation after Fourier transform to impact parameter space. Furthermore, the $t=0$ limit of $H_{v}^{q}$ is then given by the usual PDFs. In this section we explore the GPDs that are obtained from our default fit together with a model ansatz for the $\xi$ dependence.

As is well known [117, 118], GPDs admit an integral representation in terms of double distributions $k(\rho, \eta, t) !^{4}$ For valence quarks one can writ5 5

\footnotetext{
${ }^{4}$ It is common to write the arguments of the double distribution as $k(\beta, \alpha, t)$. We changed notation here because $\alpha$ and $\beta$ are already used otherwise.

${ }^{5}$ In general, the integration extends over $-1<\rho<1$ and $|\rho|-1<\eta<1-|\rho|$. Its restriction to $\rho>0$ for valence quarks has been proposed in [119.
}
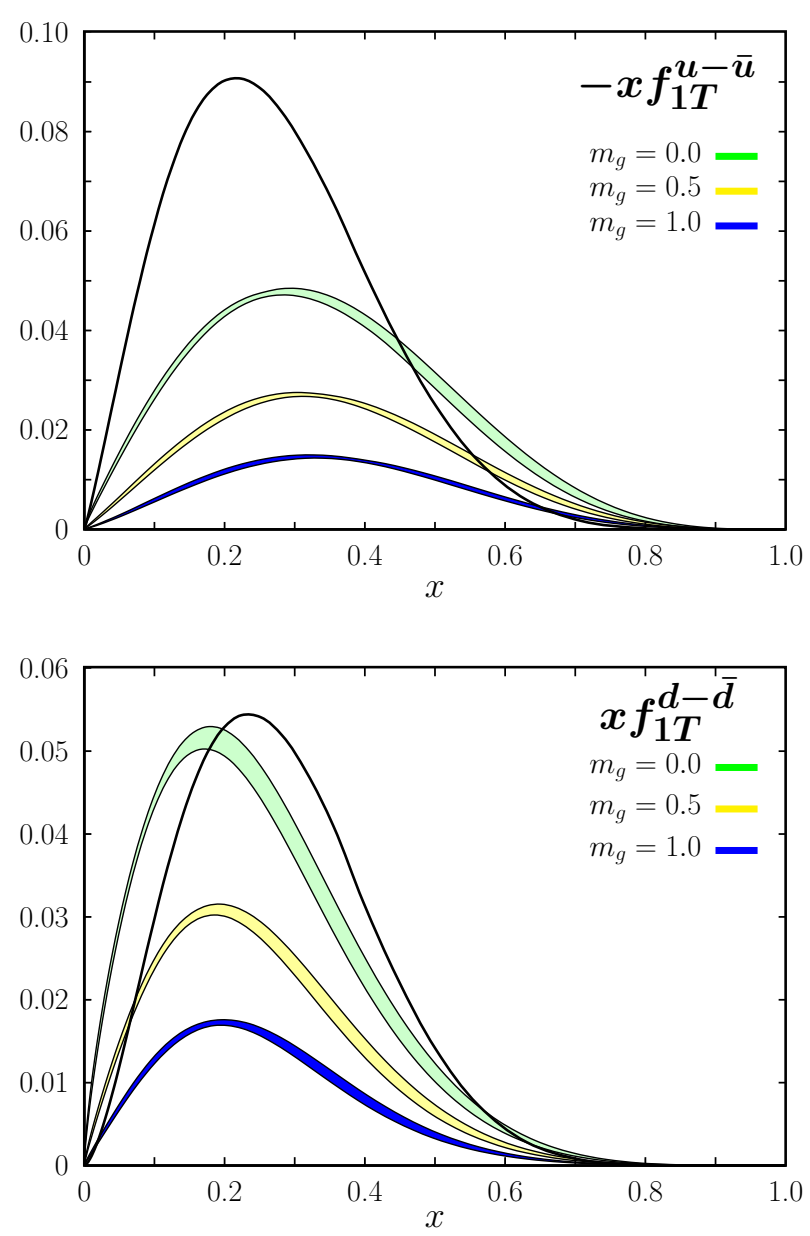

Figure 26: The first $k_{T}^{2}$ moment of the Sivers function for valence $u$ and $d$ quarks, evaluated at the scale $\mu=1 \mathrm{GeV}$ and multiplied with $x$. The bands represent the results of the model (79) together with our fit ABM 0, and the solid lines show the recent phenomenological extraction [116].

$$
\begin{aligned}
K_{v}^{q}(x, \xi, t)= & \int_{0}^{1} \mathrm{~d} \rho \int_{\rho-1}^{1-\rho} \mathrm{d} \eta \\
& \times \delta(\rho+\xi \eta-x) k_{v}^{q}(\rho, \eta, t),
\end{aligned}
$$

where $K_{v}^{q}=H_{v}^{q}, E_{v}^{q}$ as before. A useful property of this representation is that, without any restriction on the double distributions, it ensures the polynomiality property of the resulting GPDs, which is required by Lorentz covariance, see e.g. [2, 3]. From (82) it also follows that $K_{v}^{q}(x, \xi, t)=$ 0 for $x \leq-\xi$. An often used model for the double distribution, suggested long ago by Radyushkin 
and Musatov[120], is to assume

$$
k_{v}^{q}(\rho, \eta, t)=K_{v}^{q}(\rho, 0, t) w(\rho, \eta)
$$

with a weight function that generates the $\xi$ dependence of the GPDs and is normalized as

$$
\int_{\rho-1}^{1-\rho} \mathrm{d} \eta w(\rho, \eta)=1
$$

The form

$$
w(\rho, \eta)=\frac{3}{4} \frac{(1-\rho)^{2}-\eta^{2}}{(1-\rho)^{3}}
$$

has been used in many phenomenologically analyses of DVCS and exclusive meson production, see e.g. [1, 100, 121, 122].

With the help of the $\delta$ function we can perform the integral over $\eta$ in 82 . Inserting the ansatz specified by $(83)$ and 85 we obtain

$$
\begin{array}{r}
K_{v}^{q}(x, \xi, t)=\frac{3}{4 \xi^{3}} \int_{\rho_{\min }}^{\rho_{\max }} \frac{\mathrm{d} \rho}{1-\rho} K_{v}^{q}(\rho, 0, t) \\
\times\left(1+\xi-\frac{1-x}{1-\rho}\right)\left(\frac{1-x}{1-\rho}-1+\xi\right)
\end{array}
$$

with integration boundaries

$$
\begin{aligned}
& \rho_{\min }= \begin{cases}0 & \text { for } x \leq \xi, \\
x-\frac{\xi}{1-\xi}(1-x) & \text { for } x>\xi,\end{cases} \\
& \rho_{\max }=x+\frac{\xi}{1+\xi}(1-x) .
\end{aligned}
$$

The expression in the second line of 86 is zero at the integration boundaries and has its maximum at $\rho=x$.

In figure 27 we show $E_{v}^{q}$ obtained in the model just described at various values of the skewness $\xi$. The GPD $H_{v}^{q}$ looks similar in shape. We see in the figure that the valence-quark GPDs exhibit a pronounced maximum (or minimum) at a value of $x$ between 0 and $\xi$. Note that in this region the integral in 86 extends down to $\rho=0$, where $K_{v}^{q}(\rho, 0, t)$ is singular for small enough $t$ according to our discussion in section 7.1, With this behavior of the GPDs, the dominant contribution to the convolution integrals in the amplitudes of DVCS and exclusive meson production is accumulated in the vicinity of $x \approx \xi$.

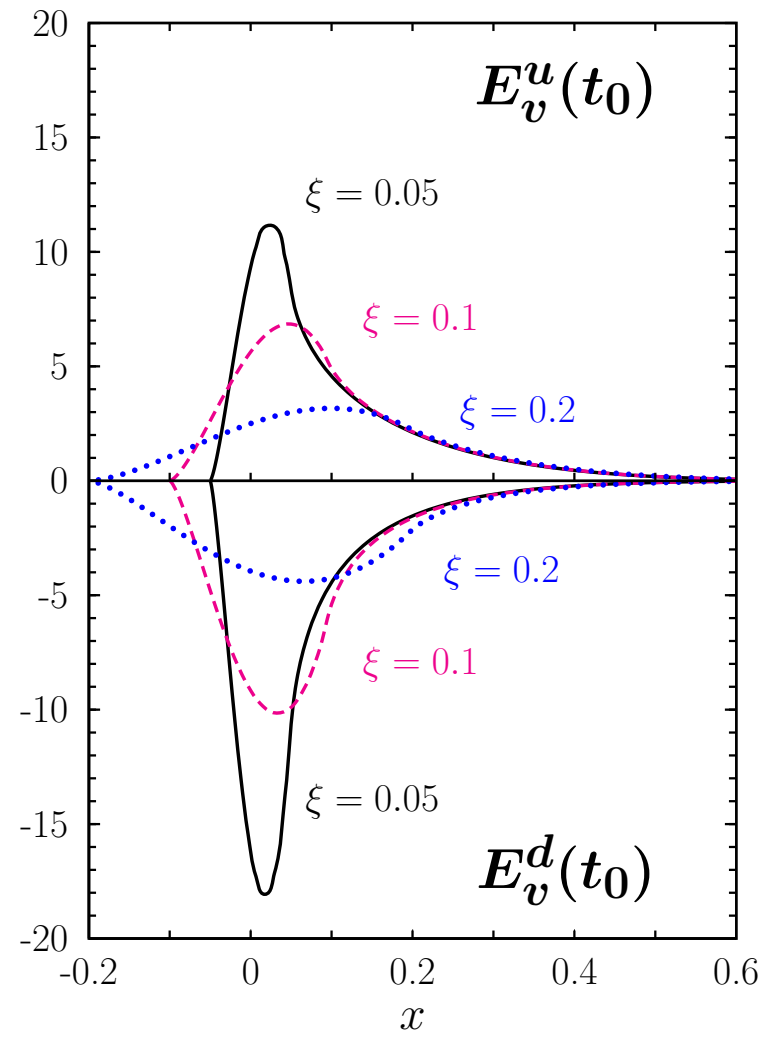

Figure 27: The valence GPD $E_{v}^{q}$ at the scale $\mu=2 \mathrm{GeV}$ for selected values of $\xi$. We set $-t$ to its minimum value $-t_{0}=4 \xi^{2} m^{2} /\left(1-\xi^{2}\right)$ that can be achieved in a physical process at given $\xi$.

Let us now discuss the behavior of the GPDs in the limit $x \rightarrow 1$ at fixed $\xi$ and $t$. For small enough $(1-x)$ the integration in $(86)$ extends over a narrow interval around $\rho=x$, so that we need to know the behavior of the zero-skewness GPDs for $x \rightarrow 1$ at fixed $t$. With our ansatz in section 6.1 we have $K_{v}^{q}(\rho, 0, t) \approx K_{v}^{q}(\rho, 0,0) \approx c(1-\rho)^{\beta}$ in that limit. Inserting this approximation in 86 , we can easily perform the $\rho$ integral and obtain

$$
\begin{gathered}
K_{v}^{q}(x, \xi, t) \approx c(1-x)^{\beta} \frac{3}{2 \beta(\beta-1)(\beta-2)} \\
\quad \times \frac{1}{\xi^{3}}\left[\frac{1+\xi(\beta-1)}{(1+\xi)^{\beta-1}}-\frac{1-\xi(\beta-1)}{(1-\xi)^{\beta-1}}\right] .
\end{gathered}
$$

For $\xi \ll 1$ this simplifies to

$$
\begin{aligned}
& K_{v}^{q}(x, \xi, t) \approx c(1-x)^{\beta} \\
& \quad \times\left[1+\frac{1}{10}(\beta+1)(\beta+2) \xi^{2}+\mathcal{O}\left(\xi^{4}\right)\right],
\end{aligned}
$$


i.e. the GPD at finite $\xi$ is larger in absolute value than in the forward limit, which it approaches smoothly. We see that in the limit $x \rightarrow 1$ our ansatz yields a $t$-independent GPD for all values of $\xi$, which (as in the case of zero skewness) is necessary for having a finite average size of the proton. Note that with the Regge-type approximation (72) for the profile function, the GPD at nonzero skewness contains a factor $\exp \left[t B_{R}\right]$ and thus remains $t$-dependent for $x \rightarrow 1$.

In the limit $x \rightarrow 1$ at fixed $\xi$ and $t$ there is a prediction that the dominant graphs describing the GPDs are such that, starting from a three-quark configuration of the proton, all longitudinal momentum is transferred to a single quark by perturbative gluon exchange [123. This generalizes the corresponding statement for the $x \rightarrow 1$ limit of the ordinary PDFs 124. The analysis in 123 predicts a power behavior

$$
\begin{aligned}
H_{v}^{q}(x, \xi, t) & \approx(1-x)^{3} a_{v}^{q}(\xi) \\
E_{v}^{q}(x, \xi, t) & \approx(1-x)^{5} b_{v}^{q}(\xi)
\end{aligned}
$$

with a dependence on $\xi$ but no dependence on $t$. The $t$-independence in this prediction is consistent with the finite-size requirement discussed above. As we see in (88), the behavior $(90)$ follows if $q_{v}(x) \sim(1-x)^{3}$ and $e_{v}^{q}(x) \sim(1-x)^{5}$ for $x \rightarrow 1$. For the valence $\operatorname{PDF} q_{v}(x)$ this is just the behavior predicted by dimensional counting.

The prediction in 90 must be taken with due caution, since among other things it does not take into account the change of the large- $x$ behavior induced by DGLAP evolution; for a more detailed discussion we refer to section 3.5 in [5]. We saw in section 6.2 that the phenomenologically extracted valence PDFs approximately follow a power law in $(1-x)$ at large $x$. As discussed there, we cannot determine the corresponding power for the mathematical limit $x \rightarrow 1$, but we can instead fit such a power for small but finite $(1-x)$. The resulting powers for $u$ quarks are typically between 3 and 4 at the scale $\mu=2 \mathrm{GeV}$ and thus not very far from the above prediction. By contrast, the effective large- $x$ power for $d$ quarks is significantly larger (except for the CT parton densities). As for $e_{v}^{q}(x)$, we find from table 12 that the large- $x$ powers obtained in our GPD fits range from 4 to
6. All in all, we do not find strong evidence for the quantitative validity of the prediction $(90)$, at least not at $\mu=2 \mathrm{GeV}$.

\section{Summary}

We have performed a detailed review of the data on electromagnetic nucleon form factors andwith an ansatz for the functional dependenceused them to determine the GPDs $H_{v}^{q}$ and $E_{v}^{q}$ for unpolarized valence quarks.

We find that the quality of the experimental data is clearly important for the quantitative extraction of GPDs. A resolution of the inconsistencies between several data sets on the ratio $R^{p}=\mu_{p} G_{E}^{p} / G_{M}^{p}$ at low $t$ (all dating from after 2000 ) is of highest urgency in this respect. We also point out inconsistencies in the data on $G_{M}^{n}$ between [20, 21] and more recent measurements at Jefferson Lab. We decided to discard the data points in [20, 21] that are in conflict with the more recent results and caution against the use of parameterizations that fit the older data. Finally, our fits show that the precision of $G_{M}^{p}$, the best known among all form factors, is of great importance for the determination of GPDs. Quantitative control over two-photon exchange contributions is crucial in this respect, and measurements of the ratio of elastic $e^{+} p$ and $e^{-} p$ cross sections [125, 126, 127. will hopefully bring further progress to this area. We find that the inclusion of the very precisely measured squared neutron charge radius has a clear impact on fits of the form factors, whereas our global fits of the nucleon form factors in the full available $t$ range are not well suited to determine with precision the charge or magnetic radii, i.e. the derivatives of form factors at $t=0$. A resolution of the discrepancies between the determinations of the proton charge radius from the Lamb shift in either electronic or muonic hydrogen would be highly welcome, as it would allow one to include this radius as an extra constraint in the determination of the form factors.

The electromagnetic nucleon form factors receive contributions from strange quarks and antiquarks, which are small but remain poorly known. Estimating their size, we find that they start to be 
quantitatively relevant at the present level of precision, but that their neglect does not change the qualitative picture. Experimentally, the strange form factors can be determined in parity violating electron-nucleon scattering, but it is not clear to us whether in the foreseeable future this can be brought to a sufficient level of precision and be extended over a significant range in $t$. Perhaps more help will come from determinations in lattice $\mathrm{QCD}$, once the present systematic uncertainties in those extractions can be brought under quantitative control. If one is willing to make a model ansatz for the GPDs, one can connect the strange Dirac form factor $F_{1}^{s}$ with the difference $s(x)-\bar{s}(x)$ of parton distributions. This difference is poorly known at present, but significant progress in their determination can be expected in forthcoming years from the production of $W$ and $Z$ bosons at the LHC.

We have used the available data on the nucleon form factors to extract an interpolated set of data for the Dirac and Pauli form factors in the quark flavor basis, i.e. $F_{1}^{u}, F_{1}^{d}, F_{2}^{u}$ and $F_{2}^{d}$. The $t$-range over which all form factors can be determined is currently limited by the measurement of the electric neutron form factor or of the ratio $R^{n}=\mu_{n} G_{E}^{n} / G_{M}^{n}$. Measurements for $-t$ above $3.4 \mathrm{GeV}^{2}$ after the $12 \mathrm{GeV}$ upgrade at Jefferson Lab will be of immediate interest in this respect. They might in particular reveal whether any of the isosinglet combinations $F_{2}^{p}+F_{2}^{n}$ or $G_{E}^{p}+G_{E}^{n}$ has a zero crossing at $-t$ around $4 \mathrm{GeV}^{2}$. Our fits strongly suggest that $G_{M}^{p}$ is dominated by $F_{1}^{u}$ at large $t$, so that $F_{1}^{u}$ may be regarded as reasonably well known up to $-t$ of order $30 \mathrm{GeV}^{2}$.

The flavor form factors we have extracted exhibit several clear trends. $F_{1}^{d}$ decreases significantly faster with $-t$ than $F_{1}^{u}$, a behavior that is naturally explained by the Feynman mechanism, given that the PDF for $d$ quarks decreases significantly faster with $x$ than the one for $u$ quarks. Interestingly, we do not see the same trend for the Pauli form factors: the ratio $F_{2}^{d} / F_{2}^{u}$ remains quite flat in the region of $-t$ up to $3.4 \mathrm{GeV}^{2}$, where we have information from the data. It will be most interesting to see in future data whether this trend continues for higher $-t$.

Using our interpolated data set, we have investi- gated several one- and two-parameter fits to individual form factors and their linear combinations. We find that a satisfactory description of all form factors up to $-t=3.4 \mathrm{GeV}^{2}$ is possible with a power-law $F(t) / F(0)=\left(1-t / M^{2}\right)^{-p}$ in the flavor basis of the Dirac and Pauli form factors. A dipole form works only well in a few cases. Using the product (16) of two power laws for each flavor form factor, we obtain an excellent global fit to all form factor data in their available $t$ range.

The current form factor data, together with a slight extension of the ansatz for GPDs developed in [5], allow for a significant advance in the determination of GPDs compared with our previous work. As we already observed in [5], the fit of the GPD is significantly constrained by the positivity requirements that arise from the relation between zero-skewness GPDs and parton densities in impact parameter space. We perform a global fit of all form factor data and find significant correlations between the different parameters describing the GPDs. Independent information about the distributions $E_{v}^{u}$ and $E_{v}^{d}$, whose forward limits are unknown, would be most helpful. In the future this might be provided by the determination of higher moments of GPDs in lattice QCD, and by measurements of exclusive processes (which are, however, described by GPDs at finite skewness and thus add a further kinematic dependence that needs to be modeled successfully). We note that the electromagnetic form factors provide indirect constraints on GPDs at high values of $t$, which will conceivably never be accessible in hard exclusive scattering processes.

We performed fits of the GPDs using several PDF sets for the forward limit of $H_{v}^{q}$ and with several settings regarding parameters and the data selection. The spread of the GPDs due to these variations is more important than the parametric errors of the individual fits. An improved knowledge of the valence PDFs, especially at small and at large $x$, will be of great use and can be expected from forthcoming measurements at the LHC or at Jefferson Lab.

Despite the present uncertainties, certain features are common to all our fits and can hence be regarded as firm results under the hypothesis that our basic ansatz for the functional form of 
the GPDs is adequate. In particular, we find that $E_{v}^{q}$ decreases faster with $x$ than $H_{v}^{q}$, although not very much for $d$ quarks. The data on $G_{E}^{n}$ and the associated squared radius $r_{n E}^{2}$ favor a small splitting between the effective Regge slopes $\alpha_{q}^{\prime}$ for $u$ and $d$ quarks, which we take to be equal in $H_{v}^{q}$ and $E_{v}^{q}$. We also find that the effective Regge intercept $\alpha_{q}$ is larger in $E_{v}^{q}$ than in $H_{v}^{q}$ in all our fits. By contrast, we cannot conclude whether the data prefers different parameters $\alpha_{q}$ in $E_{v}^{q}$ for $u$ and $d$ quarks.

From the $t$-dependence of the fitted GPDs we can compute the average squared impact parameter of valence quarks at given $x$ and find it to be clearly larger for $d$ quarks than for $u$ quarks when $x$ is above 0.3 . The sideways shift $s_{q}(x)$ in the average impact parameter induced by transverse proton polarization (which is proportional to $E_{v}^{q}$ at $t=0$ ) is found to be $0.1 \mathrm{fm}$ for $u$ quarks and $-0.17 \mathrm{fm}$ for $d$ quarks at $x=0.1$. It decreases in absolute size as $x$ increases.

Evaluating Ji's sum rule with out fitted GPDs, we can extract the total angular momentum carried by quarks of a given flavor minus the corresponding contribution from antiquarks. We find

$$
J_{v}^{u}=0.230_{-0.024}^{+0.009}, \quad J_{v}^{d}=-0.004_{-0.016}^{+0.010}
$$

at a scale $\mu=2 \mathrm{GeV}$, where the uncertainties reflect all variations we allowed in our fits. The total angular momentum carried by sea quarks cannot be determined with information from the electromagnetic form factors. We note that from current PDF determinations one finds that the nonstrange sea carries about $15 \%$ of the longitudinal proton momentum at $\mu=2 \mathrm{GeV}$.

We have used our fitted valence GPDs for unpolarized quarks to study a variety of related quantities. Assuming a simple model for the GPDs of longitudinally polarized quarks, where their $t$ dependence is taken to be equal to the unpolarized case, we can evaluate the isotriplet axial form factor $F_{A}(t)$ of the nucleon and find it to be at the lower edge of the uncertainty band from the experimental extraction of this quantity. A slower decrease with $t$ of the polarized GPDs, as well as the contribution of polarized sea quarks, could increase $F_{A}(t)$ and improve the agreement with the data, which themselves remain rather imprecise and limited in $t$. Also for this quantity, advances in lattice computations will be of great interest.

Evaluating the form factors that describe wideangle Compton scattering in the handbag approach, we obtain agreement with the data at the $30 \%$ level for $s \approx 11 \mathrm{GeV}^{2}$, which we consider to be a success for a parameter free calculation, given the uncertainties of the handbag approach itself in this kinematic region.

Combining our GPDs with a simple model for chromodynamic lensing, we can estimate the Sivers distributions for valence $u$ and $d$ quarks. Comparing the results with a recent phenomenological determination of these distributions, we find overall agreement in sign and magnitude but clear differences in the details. We think that the model of the lensing effect, which uses a simple quark-diquark picture and one-gluon exchange, is the most important source of uncertainty in this comparison.

Finally, we have combined our fitted zeroskewness GPDs with the double distribution ansatz of Musatov and Radyushkin in order to compute the valence GPDs at finite skewness. These results can be used as an input for phenomenological analyses of hard exclusive scattering processes, in particular for the kinematics of current and future fixed-target experiments.

In conclusion, the improvements in the measurement of the electromagnetic form factors over the last decade has significantly advanced our ability to perform a model-dependent extraction of the GPDs for unpolarized valence quarks. Further significant progress can be expected for the next decade from the measurement of form factors, parton densities, and hard exclusive processes and from lattice calculations. This will hopefully also enable us to get a firmer grip on the model dependence due to assuming a functional form of the GPDs.

\section{Acknowledgments}

We gratefully thank John Arrington for discussions about the proton form factors and for providing numerical values for several of his results, Mariaelena Boglione for providing numerical values of the extraction [116] of the Sivers function, 
Daniela Rohe for correspondence about the neutron form factor results [54, 55], and Thorsten Feldmann for his collaboration at an early stage of this project.

\section{A Tables of form factors}

In table 20 we give the values and errors of $R^{n}$ for the data set we have selected. Except for the entries from Plaster 05, Geis 08 and Riordan 10, which directly quote results on $R^{n}$, we have computed this ratio from $G_{E}^{n}$ and the assumed value of $G_{M}^{n}$ for the reasons explained in section 3.2 .

In table 21 we list the results for the flavor form factors we have extracted from our default data set as explained in section 5.1 .

\section{B Matrices for computing fit er- rors}

In this appendix we give the information that is needed to compute parametric errors for our default fit ABM 1 and for the power-law fit of section 3.4. A convenient proceduce to propagate errors is the so-called Hessian method used in modern PDF determinations, see e.g. 64, 128. We briefly describe this method and then list the relevant matrices.

Let us introduce the column vector $\boldsymbol{p}$ of the $n$ original fit parameters, as well as the vector of transformed parameters $\boldsymbol{z}$ defined by

$$
\boldsymbol{p}-\boldsymbol{p}_{0}=E \boldsymbol{z},
$$

where $\boldsymbol{p}_{0}$ is the set of parameters that minimizes $\chi^{2}$. The matrix $E$ satisfies

$$
E E^{T}=V
$$

with the standard covariance matrix $V$ for the parameters $\boldsymbol{p}$. The deviation of $\chi^{2}$ from its minimum value is then given by

$$
\Delta \chi^{2}=\left(\boldsymbol{p}-\boldsymbol{p}_{0}\right)^{T} V^{-1}\left(\boldsymbol{p}-\boldsymbol{p}_{0}\right)=\boldsymbol{z}^{T} \boldsymbol{z},
$$

The error on a function $f$ of the parameters, as given by linear error propagation, can be written as

$$
\begin{aligned}
\Delta f & =\sqrt{\sum_{i=1}^{n}\left[\frac{\partial f(\boldsymbol{p})}{\partial z_{i}}\right]_{\boldsymbol{p}=\boldsymbol{p}_{0}}^{2}} \\
& =\sqrt{\sum_{i=1}^{n}\left[\frac{f\left(\boldsymbol{p}_{i}^{+}\right)-f\left(\boldsymbol{p}_{i}^{-}\right)}{2}\right]^{2}}
\end{aligned}
$$

where the parameter set $\boldsymbol{p}_{i}^{ \pm}$is specified by the condition

$$
\left(\boldsymbol{p}_{i}^{ \pm}-\boldsymbol{p}_{0}\right)_{j}= \pm E_{j i} \cdot
$$

In the second step of (95) we have approximated the derivative by a difference quotient, which is consistent in the region where linear error propagation is adequate. The vector given by the $i$ th column of the matrix $E$ thus gives the amount by which the central values of the parameters need to be shifted to obtain a set of parameters on the $\Delta \chi^{2}=1$ contour.

In table 22 we give the matrix $E_{D}$ for the default GPD fit (ABM 1) and the matrix $E_{P}$ for the power law fit of section 3.4 . The order of entries in the matrices corresponds to the following vectors of parameters:

$$
\boldsymbol{p}_{D}=\left(\begin{array}{c}
A_{u} \\
A_{d} \\
B_{u} \\
B_{d} \\
C_{u} \\
C_{d} \\
D_{u} \\
D_{d} \\
\alpha_{d}^{\prime} \\
\alpha
\end{array}\right), \quad \boldsymbol{p}_{P}=\left(\begin{array}{c}
a_{1}^{u}+b_{1}^{u} \\
b_{1}^{u} \\
a_{1}^{d}+b_{1}^{d} \\
b_{1}^{d} \\
a_{2}^{u}+b_{2}^{u} \\
b_{2}^{u} \\
a_{2}^{d}+b_{2}^{d} \\
b_{2}^{d} \\
p_{1}^{u} \\
p_{1}^{d} \\
p_{2}^{u} \\
p_{2}^{d} \\
q_{1}^{u}-p_{1}^{u}
\end{array}\right) .
$$

The central values of the fit parameters are given in (50) and table 11 for the GPD fit, and those of the power-law fit are given in table 5 . 


\begin{tabular}{lccll}
\hline$-t\left[\mathrm{GeV}^{2}\right]$ & $R^{n}$ & \multicolumn{2}{c}{ error } & reference \\
& & total & stat. & \\
\hline 0.21 & 0.1108 & 0.0260 & 0.0252 & Passchier 99 \\
\hline 0.495 & 0.1334 & 0.0201 & 0.0177 & Zhu 01 \\
\hline 0.5 & 0.1517 & 0.0121 & 0.0095 & Warren 03 \\
1.0 & 0.2457 & 0.0350 & 0.0292 & \\
\hline 0.15 & 0.0706 & 0.0122 & 0.0095 & Herberg 99 \\
0.34 & 0.1485 & 0.0212 & & \\
\hline 0.30 & 0.1140 & 0.0132 & 0.0126 & Glazier 04 \\
0.59 & 0.1566 & 0.0240 & 0.0230 & \\
0.79 & 0.1992 & 0.0395 & 0.0383 & \\
\hline 0.447 & 0.1444 & 0.0175 & 0.0170 & Plaster 05 \\
1.132 & 0.2506 & 0.0218 & 0.0210 & \\
1.450 & 0.3616 & 0.0353 & 0.0344 & \\
\hline 0.35 & 0.1112 & 0.0083 & & Rohe 05 \\
\hline 0.67 & 0.1763 & 0.0258 & & Bermuth 03 \\
\hline 0.142 & 0.0505 & 0.0078 & 0.0072 & Geis 08 \\
0.203 & 0.0695 & 0.0093 & 0.0084 & \\
0.291 & 0.1022 & 0.0135 & 0.0127 & \\
0.415 & 0.1171 & 0.0189 & 0.0182 & \\
\hline 1.72 & 0.273 & 0.0361 & 0.020 & Riordan 10 \\
2.48 & 0.412 & 0.0600 & 0.048 & \\
3.41 & 0.496 & 0.0813 & 0.067 & \\
\hline & & \\
\hline & & & \\
\hline & & \\
\hline
\end{tabular}

${ }^{a}$ gives asymmetric errors; we have symmetrized by taking the maximum of the two errors

${ }^{b}$ values from Table IX, "FSI+MEC+IC+RC"

Table 20: Values of $R^{n}$ measured in polarization experiments on deuterium or ${ }^{3}$ He. References for the data sets are given in table 4 . 


\begin{tabular}{ccccc}
\hline$-t\left[\mathrm{GeV}^{2}\right]$ & $F_{1}^{u}$ & $F_{1}^{d}$ & $F_{2}^{u}$ & $F_{2}^{d}$ \\
\hline 0.039 & $1.810 \pm 0.039$ & $0.908 \pm 0.034$ & $1.471 \pm 0.059$ & $-1.833 \pm 0.056$ \\
0.088 & $1.610 \pm 0.032$ & $0.815 \pm 0.048$ & $1.251 \pm 0.044$ & $-1.565 \pm 0.071$ \\
0.142 & $1.414 \pm 0.027$ & $0.681 \pm 0.017$ & $1.104 \pm 0.036$ & $-1.352 \pm 0.043$ \\
0.156 & $1.392 \pm 0.037$ & $0.705 \pm 0.056$ & $1.044 \pm 0.045$ & $-1.347 \pm 0.070$ \\
0.203 & $1.241 \pm 0.026$ & $0.588 \pm 0.016$ & $0.972 \pm 0.033$ & $-1.133 \pm 0.038$ \\
0.243 & $1.148 \pm 0.036$ & $0.560 \pm 0.048$ & $0.857 \pm 0.040$ & $-1.092 \pm 0.052$ \\
0.291 & $1.023 \pm 0.016$ & $0.475 \pm 0.014$ & $0.798 \pm 0.030$ & $-0.948 \pm 0.049$ \\
0.300 & $1.020 \pm 0.016$ & $0.478 \pm 0.014$ & $0.780 \pm 0.054$ & $-0.937 \pm 0.103$ \\
0.340 & $0.979 \pm 0.018$ & $0.468 \pm 0.019$ & $0.696 \pm 0.039$ & $-0.890 \pm 0.068$ \\
0.350 & $0.924 \pm 0.051$ & $0.416 \pm 0.026$ & $0.719 \pm 0.061$ & $-0.832 \pm 0.065$ \\
0.415 & $0.862 \pm 0.014$ & $0.375 \pm 0.015$ & $0.626 \pm 0.028$ & $-0.740 \pm 0.046$ \\
0.447 & $0.831 \pm 0.012$ & $0.368 \pm 0.013$ & $0.577 \pm 0.025$ & $-0.711 \pm 0.041$ \\
0.495 & $0.759 \pm 0.011$ & $0.320 \pm 0.013$ & $0.523 \pm 0.021$ & $-0.643 \pm 0.036$ \\
0.500 & $0.758 \pm 0.010$ & $0.327 \pm 0.009$ & $0.513 \pm 0.020$ & $-0.648 \pm 0.035$ \\
0.590 & $0.668 \pm 0.010$ & $0.272 \pm 0.013$ & $0.445 \pm 0.018$ & $-0.564 \pm 0.033$ \\
0.623 & $0.640 \pm 0.010$ & $0.259 \pm 0.014$ & $0.413 \pm 0.016$ & $-0.546 \pm 0.028$ \\
0.670 & $0.612 \pm 0.010$ & $0.243 \pm 0.012$ & $0.378 \pm 0.016$ & $-0.513 \pm 0.026$ \\
0.790 & $0.524 \pm 0.010$ & $0.198 \pm 0.015$ & $0.326 \pm 0.014$ & $-0.421 \pm 0.023$ \\
1.000 & $0.438 \pm 0.014$ & $0.160 \pm 0.012$ & $0.244 \pm 0.016$ & $-0.310 \pm 0.018$ \\
1.132 & $0.372 \pm 0.008$ & $0.124 \pm 0.007$ & $0.223 \pm 0.010$ & $-0.255 \pm 0.014$ \\
1.177 & $0.372 \pm 0.009$ & $0.132 \pm 0.015$ & $0.191 \pm 0.010$ & $-0.262 \pm 0.018$ \\
1.401 & $0.279 \pm 0.012$ & $0.080 \pm 0.017$ & $0.170 \pm 0.013$ & $-0.188 \pm 0.018$ \\
1.450 & $0.279 \pm 0.008$ & $0.090 \pm 0.007$ & $0.155 \pm 0.009$ & $-0.189 \pm 0.010$ \\
1.644 & $0.247 \pm 0.012$ & $0.071 \pm 0.018$ & $0.125 \pm 0.012$ & $-0.149 \pm 0.018$ \\
1.720 & $0.231 \pm 0.011$ & $0.058 \pm 0.007$ & $0.118 \pm 0.011$ & $-0.131 \pm 0.008$ \\
2.480 & $0.139 \pm 0.003$ & $0.028 \pm 0.004$ & $0.062 \pm 0.004$ & $-0.072 \pm 0.005$ \\
3.410 & $0.087 \pm 0.002$ & $0.013 \pm 0.003$ & $0.035 \pm 0.002$ & $-0.037 \pm 0.003$ \\
\hline & & & & \\
& & & \\
0.96 &
\end{tabular}

Table 21: The flavor form factors we obtain by interpolation of the data, as explained in section 5.1 . 


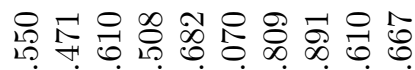

令藅

숭 0

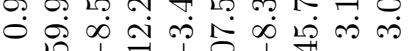
I

車年

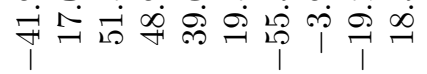

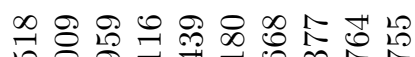

元

๑

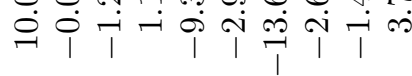

유유

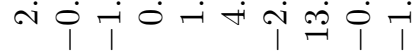

स゙ 官

귱ำ

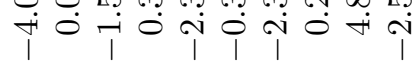

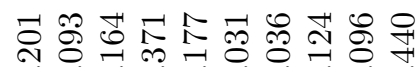
-

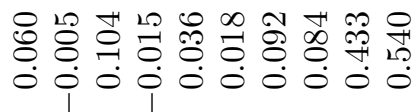

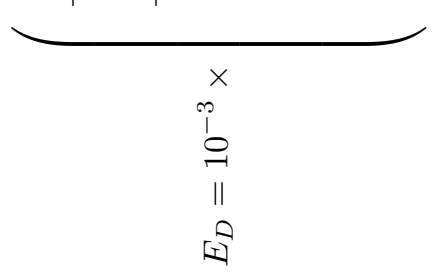

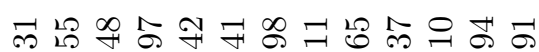

क सं

จิ

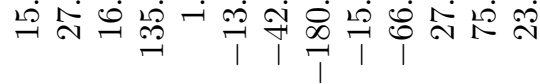

岀

ஜ்

బ

ஷ่

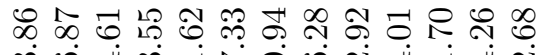

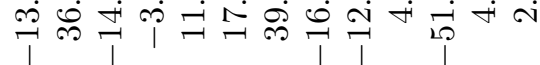

\#

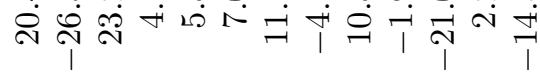

舟

i

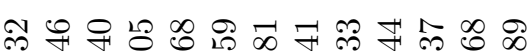

○े

बำ

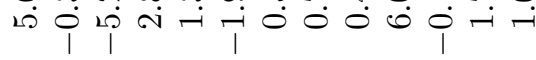

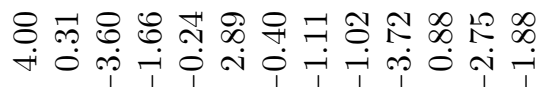

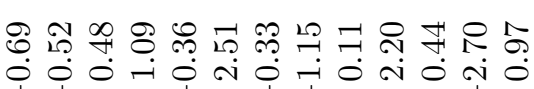

$\dot{0} \dot{0} \dot{0}-\dot{0}$ )

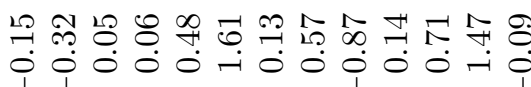

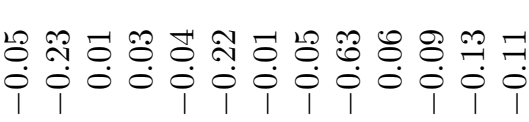

ஜேं

II

[1] 


\section{References}

[1] K. Goeke, M. V. Polyakov and M. Vanderhaeghen, Prog. Part. Nucl. Phys. 47 (2001) 401 [hep-ph/0106012].

[2] M. Diehl, Phys. Rept. 388 (2003) 41 [hep-ph/0307382].

[3] A. V. Belitsky and A. V. Radyushkin, Phys. Rept. 418 (2005) 1 [hep-ph/0504030].

[4] S. Boffi and B. Pasquini, Riv. Nuovo Cim. 30 (2007) 387 [arXiv:0711.2625].

[5] M. Diehl, T. Feldmann, R. Jakob and P. Kroll, Eur. Phys. J. C 39, 1 (2005) [hep-ph/0408173].

[6] J. J. Kelly, Phys. Rev. C 66 (2002) 065203 [hep-ph/0204239].

[7] H. Gao, nucl-ex/0411015.

[8] J. Friedrich and T. Walcher, Eur. Phys. J. A 17 (2003) 607 [hep-ph/0303054].

[9] J. Arrington, W. Melnitchouk and J. A. Tjon, Phys. Rev. C 76 (2007) 035205 [arXiv:0707.1861].

[10] B. D. Milbrath et al. [Bates FPP Collaboration], Phys. Rev. Lett. 80 (1998) 452 [Erratum-ibid. 82 (1999) 2221] [nucl-ex/9712006].

[11] T. Pospischil et al. [A1 Collaboration], Eur. Phys. J. A 12 (2001) 125.

[12] O. Gayou et al. [Jefferson Lab Hall A Collaboration], Phys. Rev. C 64 (2001) 038202.

[13] O. Gayou et al. [Jefferson Lab Hall A Collaboration], Phys. Rev. Lett. 88 (2002) 092301 [nuclex/0111010].

[14] V. Punjabi et al. [Jefferson Lab Hall A Collaboration], Phys. Rev. C 71 (2005) 055202 [Erratumibid. C 71 (2005) 069902] [nucl-ex/0501018].

[15] G. MacLachlan et al., Nucl. Phys. A 764 (2006) 261.

[16] A. J. R. Puckett et al., Phys. Rev. Lett. 104 (2010) 242301 [arXiv:1005.3419].

[17] M. Paolone et al., Phys. Rev. Lett. 105 (2010) 072001 [arXiv:1002.2188].

[18] G. Ron et al. [Jefferson Lab Hall A Collaboration], Phys. Rev. C 84 (2011) 055204 [arXiv:1103.5784].

[19] X. Zhan et al., Phys. Lett. B 705 (2011) 59 [arXiv:1102.0318].

[20] H. Anklin et al., Phys. Lett. B 428 (1998) 248.

[21] G. Kubon et al., Phys. Lett. B 524 (2002) 26 [nucl-ex/0107016].

[22] H. Anklin et al., Phys. Lett. B 336 (1994) 313.

[23] J. Lachniet et al. [CLAS Collaboration], Phys. Rev. Lett. 102 (2009) 192001 [arXiv:0811.1716].

[24] B. Anderson et al. [Jefferson Lab E95-001 Collaboration], Phys. Rev. C 75 (2007) 034003 [nuclex/0605006].

[25] C. Herberg et al., Eur. Phys. J. A 5 (1999) 131. 
[26] D. I. Glazier et al., Eur. Phys. J. A 24 (2005) 101 [nucl-ex/0410026].

[27] B. Plaster et al. [Jefferson Lab E93-038 Collaboration], Phys. Rev. C 73 (2006) 025205 [nuclex/0511025].

[28] I. Passchier et al., Phys. Rev. Lett. 82 (1999) 4988 [nucl-ex/9907012].

[29] H. Zhu et al. [Jefferson Lab E93-026 Collaboration], Phys. Rev. Lett. 87 (2001) 081801 [nuclex/0105001].

[30] G. Warren et al. [Jefferson Lab E93-026 Collaboration], Phys. Rev. Lett. 92 (2004) 042301 [nucl-ex/0308021].

[31] E. Geis et al. [BLAST Collaboration], Phys. Rev. Lett. 101 (2008) 042501 [arXiv:0803.3827].

[32] J. Bermuth et al., Phys. Lett. B 564 (2003) 199 [nucl-ex/0303015].

[33] D. Rohe, private communication (2005).

[34] S. Riordan et al., Phys. Rev. Lett. 105 (2010) 262302 [arXiv:1008.1738].

[35] R. Schiavilla and I. Sick, Phys. Rev. C 64 (2001) 041002 [nucl-ex/0107004].

[36] J. Beringer et al. [Particle Data Group Collaboration], Phys. Rev. D 86 (2012) 010001.

[37] P. A. M. Guichon and M. Vanderhaeghen, Phys. Rev. Lett. 91 (2003) 142303 [hep-ph/0306007].

[38] J. Arrington, P. G. Blunden and W. Melnitchouk, Prog. Part. Nucl. Phys. 66 (2011) 782 [arXiv:1105.0951].

[39] J. Arrington, Phys. Rev. C 71 (2005) 015202 [hep-ph/0408261].

[40] W. M. Alberico, S. M. Bilenky, C. Giunti and K. M. Graczyk, Phys. Rev. C 79 (2009) 065204 [arXiv:0812.3539].

[41] I. A. Qattan, A. Alsaad and J. Arrington, Phys. Rev. C 84 (2011) 054317 [arXiv:1109.1441].

[42] I. A. Qattan and J. Arrington, arXiv:1209.0683.

[43] G. Ron et al. [Jefferson Lab Hall A Collaboration], Phys. Rev. Lett. 99 (2007) 202002 [arXiv:0706.0128].

[44] M. K. Jones et al. [Resonance Spin Structure Collaboration], Phys. Rev. C 74 (2006) 035201 [nucl-ex/0606015].

[45] C. B. Crawford et al., Phys. Rev. Lett. 98 (2007) 052301 [nucl-ex/0609007].

[46] W. Xu et al., Phys. Rev. Lett. 85 (2000) 2900 [nucl-ex/0008003].

[47] W. Xu et al. [Jefferson Lab E95-001 Collaboration], Phys. Rev. C 67 (2003) 012201 [nuclex/0208007].

[48] H. Gao, Nucl. Phys. A 631 (1998) 170.

[49] H. Gao et al., Phys. Rev. C 50 (1994) 546.

[50] J. J. Kelly, Phys. Rev. C 70 (2004) 068202. 
[51] M. Ostrick et al., Phys. Rev. Lett. 83 (1999) 276.

[52] R. Madey et al. [Jefferson Lab E93-038 Collaboration], Phys. Rev. Lett. 91 (2003) 122002 [nucl-ex/0308007].

[53] D. Rohe et al., Phys. Rev. Lett. 83 (1999) 4257.

[54] J. Becker et al., Eur. Phys. J. A 6 (1999) 329.

[55] J. Golak, G. Ziemer, H. Kamada, H. Witała and W. Glöckle, Phys. Rev. C 63 (2001) 034006 [nucl-th/0008008].

[56] F. E. Maas et al. [A4 Collaboration], Phys. Rev. Lett. 93 (2004) 022002 [nucl-ex/0401019].

[57] K. A. Aniol et al. [HAPPEX Collaboration], Phys. Rev. C 69 (2004) 065501 [nucl-ex/0402004].

[58] F. E. Maas et al. [A4 Collaboration], Phys. Rev. Lett. 94 (2005) 152001 [nucl-ex/0412030].

[59] K. A. Aniol et al. [HAPPEX Collaboration], Phys. Rev. Lett. 96 (2006) 022003 [nuclex/0506010].

[60] K. A. Aniol et al. [HAPPEX Collaboration], Phys. Lett. B 635 (2006) 275 [nucl-ex/0506011].

[61] A. Acha et al. [HAPPEX collaboration], Phys. Rev. Lett. 98 (2007) 032301 [nucl-ex/0609002].

[62] S. Baunack et al., Phys. Rev. Lett. 102 (2009) 151803 [arXiv:0903.2733].

[63] D. Androić et al. [G0 Collaboration], Phys. Rev. Lett. 104 (2010) 012001 [arXiv:0909.5107].

[64] A. D. Martin, W. J. Stirling, R. S. Thorne and G. Watt, Eur. Phys. J. C 63, 189 (2009) [arXiv:0901.0002].

[65] R. D. Ball et al. [NNPDF Collaboration], Nucl. Phys. B 855 (2012) 608 [arXiv:1108.1758].

[66] D. B. Leinweber et al., Phys. Rev. Lett. 94, 212001 (2005) [hep-lat/0406002].

[67] D. B. Leinweber et al., Phys. Rev. Lett. 97, 022001 (2006) [hep-lat/0601025].

[68] H. -W. Lin, arXiv:0707.3844.

[69] P. Wang, D. B. Leinweber, A. W. Thomas and R. D. Young, Phys. Rev. C 79 (2009) 065202 [arXiv:0807.0944].

[70] T. Doi et al., Phys. Rev. D 80 (2009) 094503 [arXiv:0903.3232].

[71] M. Diehl, T. Feldmann and P. Kroll, Phys. Rev. D 77 (2008) 033006 [arXiv:0711.4304].

[72] H. Dahiya and M. Gupta, Phys. Rev. D 78, 014001 (2008) [arXiv:0806.0692].

[73] A. Kiswandhi, H. C. Lee and S. N. Yang, Phys. Lett. B 704 (2011) 373 [arXiv:1107.3072].

[74] D. O. Riska and B. S. Zou, AIP Conf. Proc. 842 (2006) 303.

[75] W. Grein and P. Kroll, Nucl. Phys. A 338 (1980) 332.

[76] P. Kroll, AIP Conf. Proc. 904 (2007) 76 [hep-ph/0612026]. 
[77] P. Kroll, arXiv:0710.2771.

[78] G. D. Cates, C. W. de Jager, S. Riordan and B. Wojtsekhowski, Phys. Rev. Lett. 106 (2011) 252003 [arXiv:1103.1808].

[79] M. Burkardt, Int. J. Mod. Phys. A 18 (2003) 173 [hep-ph/0207047].

[80] D. de Florian, R. Sassot, M. Stratmann and W. Vogelsang, Phys. Rev. D 80 (2009) 034030 [arXiv:0904.3821].

[81] M. Burkardt, Phys. Lett. B 582 (2004) 151 [hep-ph/0309116].

[82] http://lhapdf.hepforge.org

[83] S. Alekhin, J. Blümlein and S. Moch, Phys. Rev. D 86 (2012) 054009 [arXiv:1202.2281].

[84] H. L. Lai et al., Phys. Rev. D 82 (2010) 074024 [arXiv:1007.2241].

[85] M. Glück, P. Jimenez-Delgado and E. Reya, Eur. Phys. J. C 53 (2008) 355 [arXiv:0709.0614].

[86] https://www.desy.de/h1zeus/combined_results/herapdftable

[87] J. Pumplin et al., JHEP 0207 (2002) 012 [hep-ph/0201195].

[88] J. C. Bernauer et al. [A1 Collaboration], Phys. Rev. Lett. 105 (2010) 242001 [arXiv:1007.5076].

[89] R. Pohl, R. Gilman, G. A. Miller and K. Pachucki, arXiv:1301.0905.

[90] R. Pohl et al., Nature 466 (2010) 213.

[91] S. D. Drell and T. M. Yan, Phys. Rev. Lett. 24 (1970) 181.

[92] J. D. Bratt et al. [LHPC Collaboration], Phys. Rev. D 82 (2010) 094502 [arXiv:1001.3620].

[93] Ph. Hägler, Phys. Rept. 490 (2010) 49 [arXiv:0912.5483].

[94] G. S. Bali et al., Phys. Rev. D 86 (2012) 054504 [arXiv:1207.1110].

[95] J. R. Green et al., arXiv:1209.1687.

[96] X. D. Ji, Phys. Rev. Lett. 78 (1997) 610 [hep-ph/9603249].

[97] A. Bacchetta and M. Radici, Phys. Rev. Lett. 107 (2011) 212001 [arXiv:1107.5755].

[98] A. Bacchetta and M. Radici, PoS QNP 2012 (2012) 041 [arXiv:1206.2565].

[99] R. L. Jaffe, hep-ph/9602236.

[100] S. V. Goloskokov and P. Kroll, Eur. Phys. J. C 53 (2008) 367 [arXiv:0708.3569].

[101] V. Bernard, L. Elouadrhiri and U. G. Meissner, J. Phys. G 28 (2002) R1 [hep-ph/0107088].

[102] T. Kitagaki et al., Phys. Rev. D 28 (1983) 436.

[103] A. V. Radyushkin, Phys. Rev. D 58 (1998) 114008 [hep-ph/9803316].

[104] M. Diehl, T. Feldmann, R. Jakob and P. Kroll, Eur. Phys. J. C 8 (1999) 409 [hep-ph/9811253]. 
[105] A. Danagoulian et al. [Jefferson Lab Hall A Collaboration], Phys. Rev. Lett. 98 (2007) 152001 [nucl-ex/0701068].

[106] H. W. Huang, P. Kroll and T. Morii, Eur. Phys. J. C 23 (2002) 301 [Erratum-ibid. C 31 (2003) 279] [hep-ph/0110208].

[107] M. Diehl, T. Feldmann, H. W. Huang and P. Kroll, Phys. Rev. D 67 (2003) 037502 [hep$\mathrm{ph} / 0212138]$.

[108] M. Diehl, T. Feldmann, R. Jakob and P. Kroll, Phys. Lett. B 460 (1999) 204 [hep-ph/9903268].

[109] D. J. Hamilton et al. [Jefferson Lab Hall A Collaboration], Phys. Rev. Lett. 94 (2005) 242001 [nucl-ex/0410001].

[110] S. J. Brodsky, D. S. Hwang and I. Schmidt, Phys. Lett. B 530 (2002) 99 [hep-ph/0201296].

[111] J. C. Collins, Phys. Lett. B 536 (2002) 43 [hep-ph/0204004].

[112] M. Burkardt, Nucl. Phys. A 735 (2004) 185 [hep-ph/0302144].

[113] S. Meissner, A. Metz and K. Goeke, Phys. Rev. D 76 (2007) 034002 [hep-ph/0703176].

[114] L. Gamberg and M. Schlegel, AIP Conf. Proc. 1374 (2011) 309 [arXiv:1012.3395].

[115] A. Bacchetta, U. D'Alesio, M. Diehl and C. A. Miller, Phys. Rev. D 70 (2004) 117504 [hep$\mathrm{ph} / 0410050]$.

[116] M. Anselmino, M. Boglione and S. Melis, Phys. Rev. D 86 (2012) 014028 [arXiv:1204.1239].

[117] D. Müller, D. Robaschik, B. Geyer, F. M. Dittes and J. Hořejši, Fortsch. Phys. 42 (1994) 101 [hep-ph/9812448].

[118] A. V. Radyushkin, Phys. Lett. B 449 (1999) 81 [hep-ph/9810466].

[119] A. V. Belitsky, D. Müller and A. Kirchner, Nucl. Phys. B 629 (2002) 323 [hep-ph/0112108].

[120] I. V. Musatov and A. V. Radyushkin, Phys. Rev. D 61 (2000) 074027 [hep-ph/9905376].

[121] M. Diehl, W. Kugler, A. Schäfer and C. Weiss, Phys. Rev. D 72 (2005) 034034 [Erratum-ibid. D 72 (2005) 059902] [hep-ph/0506171].

[122] S. V. Goloskokov and P. Kroll, Eur. Phys. J. C 59 (2009) 809 [arXiv:0809.4126].

[123] F. Yuan, Phys. Rev. D 69 (2004) 051501 [hep-ph/0311288].

[124] A. H. Mueller, Phys. Rept. 73 (1981) 237.

[125] J. Arrington et al., nucl-ex/0408020.

[126] Jefferson Lab Experiment E07-005, http://www.jlab.org/exp_prog/generated/apphallb.html

[127] M. Kohl [OLYMPUS Collaboration], AIP Conf. Proc. 1374 (2011) 527.

[128] J. Pumplin et al., Phys. Rev. D 65 (2001) 014013 [hep-ph/0101032]. 